\title{
Redox Regulation of Mitochondrial Function
}

\author{
Diane E. Handy and Joseph Loscalzo
}

\begin{abstract}
Redox-dependent processes influence most cellular functions, such as differentiation, proliferation, and apoptosis. Mitochondria are at the center of these processes, as mitochondria both generate reactive oxygen species (ROS) that drive redox-sensitive events and respond to ROS-mediated changes in the cellular redox state. In this review, we examine the regulation of cellular ROS, their modes of production and removal, and the redoxsensitive targets that are modified by their flux. In particular, we focus on the actions of redox-sensitive targets that alter mitochondrial function and the role of these redox modifications on metabolism, mitochondrial biogenesis, receptor-mediated signaling, and apoptotic pathways. We also consider the role of mitochondria in modulating these pathways, and discuss how redox-dependent events may contribute to pathobiology by altering mitochondrial function. Antioxid. Redox Signal. 16, 1323-1367.
\end{abstract}

I. Introduction $\quad 1324$

II. Cellular ROS, Redox, and Antioxidant Systems 1324

A. Sources of cellular ROS 1324

B. Mitochondrial generation of ROS $\quad 1325$

C. Redox and antioxidant systems $\quad 1326$

$\begin{array}{ll}\text { 1. SODs } & 1327\end{array}$

2. Catalase and NADPH 1328

3. GPx, reduced glutathione, Grxs, and glutathione reductase 1328

4. Prxs, Trx, and TrxR 1330

5. Cytosolic and mitochondrial NADPH 1332

D. Redox-active Cys 1332

1. Cys oxidation states and functional modification of protein thiols 1332

2. Protein S-glutathiolation 1333

3. Protein S-nitrosation 1334

4. Mitochondrial function and the thiol redox state 1334

5. Mitochondrial disulfide-relay system 1335

E. Metabolism, NADH/NAD ${ }^{+}$, and NADPH/NADP ${ }^{+} \quad 1335$

1. Regulation of glycolysis 1335

2. SIRT, $\mathrm{NAD}^{+}$, and metabolic regulation $\quad 1336$

III. Functional Consequences of Redox Modifiers 1337

$\begin{array}{ll}\text { A. } \mathrm{NO}^{\bullet} & 1337\end{array}$

1. Apoptosis and $\mathrm{NO}^{\bullet} \quad 1337$

2. $\mathrm{NO}^{\bullet}$ and mitochondrial fission and fusion 1338

3. $\mathrm{NO}^{\bullet}$ and mitochondrial respiration $\quad 1338$

B. Reactive oxygen species $\quad 1339$

1. NADPH activation of mitochondrial ROS 1339

2. Other ROS-mediated ROS generators 1339

3. Extracellular redox state and mitochondrial function 1340

4. Mitochondrial ROS and receptor-mediated signaling 1340

5. Mitochondrial respiration, inner mitochondrial membrane potential, and ROS 1341

6. UCPs and mitochondrial function $\quad 1341$

Reviewing Editors: Hossein Ardehali, Enrique Cadenas, Martha Carraway, Thomas Kietzmann, Judith Haendler, Christopher Horst Lillig, Wolfgang Maret, Pier Mastroberardino and Rodrigue Rossignol

Cardiovascular Division, Department of Medicine, Brigham and Women's Hospital, Harvard Medical School, Boston, Massachusetts. 
IV. Redox Regulation of Mitochondrial Turnover 1342

A. Mitochondrial biogenesis 1342

$\begin{array}{ll}\text { 1. Overview of biogenesis } & 1342\end{array}$

2. ROS and biogenesis 1342

3. Telomere dysfunction and mitochondrial biogenesis 1343

B. ROS and mitophagy versus apoptosis 1343

C. Heme oxygenase, carbon monoxide, and mitochondrial function 1344

V. Redox Regulation of Apoptosis 1344

A. ASK1 and Trx-mediated regulation of JNK

B. MPT pore and cell death $\quad 1345$

1. Mitochondrial permeability transition 1345

2. Mitochondrial $\mathrm{Ca}^{2+}$, redox, and mitochondrial dysfunction 1346

3. STAT3 and mitochondrial function 1346

C. Lipid oxidation and apoptotic signaling 1347

1. Cardiolipin oxidation and apoptosis 1347

2. Membrane phospholipids and AIF-mediated cell death 1347

VI. Mitochondrial Responses to Hypoxia 1348

VII. Conclusion 1349

\section{Introduction}

M ITOCHONDRIA ARE ESSENTIAL REGULATORS of cellular bioenergetics that respond to changes in environmental stimuli, such as hormones, nutrients, and oxygen tension (47, $94,99,109,290,301,385)$. Redox-sensitive cellular targets play a role in these processes, providing sensitive and rapid responses to changes in metabolism and fluxes of reactive oxygen species (ROS). Until recently, ROS have been studied for their harmful effects on proteins, lipids, and DNA that lead to cell damage and death; however, there is a growing appreciation of the role of ROS in modulating cellular processes through redox-dependent signaling $(78,169,342,349,350)$. Here, we review redox-dependent pathways that alter mitochondrial function, examine how mitochondrial function contributes to the essential production of ROS necessary for redox-dependent cellular processes, and discuss the role of mitochondrial ROS in hypoxia and apoptosis.

It is well established that mitochondria produce ROS as a consequence of electron leak during respiration $(223,349)$. Often this leak is referred to as a byproduct of respiration; however, lack of mitochondrial oxidant production leads to diminished cell signaling in response to growth factors, such as insulin, and decreases the formation of necessary protein disulfides $(169,264,284,450,464)$. Although some increases in ROS can stimulate these essential pathways, there is a threshold range of concentrations beyond which ROS become harmful $(83,206,349)$. Additionally, ROS-damaged mitochondria tend to produce more ROS, thereby activating mitochondrial-mediated apoptotic or necrotic pathways (120, 416). Adaptive antioxidant systems are essential for maintaining the critical redox balance in cells, as are mitochondrial adaptations to changes in ROS flux. Thus, in this review, we highlight the role of key intracellular antioxidant systems and discuss how they may modulate mitochondrial function. We also discuss redox modifications that are essential for normal cellular function and survival, such as the reversible protein thiol oxidation (thiol redox switches) that mediates cell signaling, and discuss the role of mitochondria in these pathways. Additionally, we consider the role of mitochondria in maladaptive responses that lead to cell death, many of which involve mitochondrial injury or dysfunction leading to mitochondrial-dependent apoptosis and necrosis.

\section{Cellular ROS, Redox, and Antioxidant Systems}

ROS are crucial modulators of cellular function. At low concentrations, ROS are essential participants in cell signaling $(136,323,450)$, whereas excess ROS can disrupt normal cellular function and promote damage to cellular lipids, nucleic acids, and proteins. Thus, a balance between ROS production and their removal allows for normal cellular function, whereas an imbalance causes oxidative stress with pathobiological consequences.

\section{A. Sources of cellular ROS}

Most cellular ROS are partially reduced forms of molecular oxygen and their derivatives and originate from the oneelectron reduction reaction yielding superoxide anion $\left(\mathrm{O}_{2}^{\bullet-}\right)$. This incomplete reduction reaction occurs as a result of electron leak during normal respiration in the mitochondrial electron transport chain (ETC) complexes or by enzymatic reduction of molecular oxygen $(249,399)$. Xanthine/xanthine oxidase, uncoupled nitric oxide synthases (NOS), cytochrome $\mathrm{P}-450$ isoforms, and NADPH-dependent oxidases (NOXs) are among the enzymes capable of producing $\mathrm{O}_{2}^{\bullet-}$. The $\alpha$ ketoglutarate dehydrogenase ( $\alpha-\mathrm{KGDH})$ complex may also provide a source of mitochondrial $\mathrm{O}_{2}^{--}$production (401). Superoxide is known to combine rapidly with nitric oxide $\left(\mathrm{NO}^{\circ}\right)$ at an approximately diffusion-limited rate $\left(k=6.7 \times 10^{9}\right.$ $M^{-1} \mathrm{~S}^{-1}$ ) to form peroxynitrite (a reactive nitrogen species [RNS] that can lead to the formation of other reactive compounds) (410). Superoxide can also become protonated to form the hydroperoxyl radical that can promote lipid peroxidation (7) and subsequent oxidative modification of proteins, low-molecular-weight thiols, and membrane lipids. Alternatively, $\mathrm{O}_{2}^{\bullet-}$ can be reduced to hydrogen peroxide, either spontaneously $\left(k=2 \times 10^{5} \mathrm{M}^{-1} \mathrm{~S}^{-1}\right.$, under physiological conditions) or through the action of superoxide dismutases (SODs), which increase the rate of dismutation $(71,260)$. In addition to its formation downstream of $\mathrm{O}_{2}^{\bullet-}$, hydrogen peroxide may also be produced directly by enzymes such as xanthine/xanthine 
oxidase and NOX4 through a flavin-dependent two-electron reduction of molecular oxygen $(38,118,211)$.

Owing to its anionic charge, $\mathrm{O}_{2}^{\bullet-}$ does not readily traverse intracellular compartments, although studies with isolated mitochondria provide evidence that superoxide leaks from mitochondria via the voltage-dependent anion channel (VDAC) (168). More recent data from mutant yeast strains lacking VDAC indicate that the translocase of the outer membrane complex participates in superoxide release from mitochondria in the absence of VDAC (55). Together, these studies suggest that although most of superoxide's effects are localized to its sites of production, transmembrane proteins can mediate $\mathrm{O}_{2}^{\bullet-}$ release from mitochondria. There have been no reliable estimates of the extent to which $\mathrm{O}_{2}^{\circ-}$ leak contributes to cytosolic oxidant levels compared to the direct release of hydrogen peroxide from mitochondria; however, in contrast to $\mathrm{O}_{2}^{--}$, the neutrally charged and less reactive hydrogen peroxide has a longer half-life and can readily diffuse across membranes (450). Given these and other properties, hydrogen peroxide plays an essential role in redox signaling. For example, it coordinates cellular responses to oxidant production, in part, by the reversible oxidation of protein thiols (see below). In excess, however, hydrogen peroxide can overoxidize protein thiols, react with free metals to form ${ }^{\bullet} \mathrm{OH}$, cause lipid peroxidation, and contribute to cellular oxidative stress $(127,324)$.

\section{B. Mitochondrial generation of ROS}

As discussed in more detail below, mitochondrial responses to ROS can increase mitochondrial production of ROS, in part, by increasing electron leak. Within mitochondria, the components of the ETC reside on the inner membrane (Fig. 1). During respiration, electrons are transferred to molecular oxygen via the four complexes of the ETC to generate water while pumping protons $\left(\mathrm{H}^{+}\right)$into the intermembrane space at complex I (NADH dehydrogenase), complex III (cytochrome c reductase), and complex IV (cytochrome c oxidase). This gradient of $\mathrm{H}^{+}$is the major contributor to the mitochondrial inner membrane potential $(\Delta \Psi)$; the return flux of protons into the matrix through the ATP synthase complex (proton motive force) powers the synthesis of ATP from ADP and inorganic phosphate. During respiration, $\sim 1 \%-2 \%$ of molecular oxygen is converted to $\mathrm{O}_{2}^{--}$due to electron leak at either complex I or complex III $(223,349)$. According to thermodynamic calculations, many of the complexes in the ETC are capable of converting molecular oxygen to $\mathrm{O}_{2}^{\bullet-}$; however, the efficiency of the transfer of electrons in the respiratory chain reduces the leak at most sites [(403) and references therein]. Some estimates indicate that $70 \%-80 \%$ of mitochondrial $\mathrm{O}_{2}^{\cdot-}$ arises from the $\mathrm{Q}$ cycle (ubiquinol, $\mathrm{QH}_{2}$, to ubiquinone, $\mathrm{Q}$ ) as part of the electron transfer to cytochrome c catalyzed by complex III (70). During this cycle, $\mathrm{O}_{2}^{\bullet-}$ can be released into the mitochondrial matrix or intermembrane space owing to the bifacial localization of complex III Qbinding sites within the inner membrane. Other reports suggest that the majority of mitochondrial $\mathrm{O}_{2}^{--}$comes from complex I during oxidation of NADH to $\mathrm{NAD}^{+}$. The actual role of complex I or III in $\mathrm{O}_{2}^{\bullet-}$ flux may depend on multiple factors, including cell type and metabolic state. For example, excess $\mathrm{NAD}^{+}$reduction (increased $\mathrm{NADH} / \mathrm{NAD}^{+}$ratio) enhances $\mathrm{O}_{2}^{\bullet-}$ generation from complex I via NADH-ubiquinone reductase activity (413). Electrons enter the ETC as $\mathrm{NADH}$ in complex I, where it is reduced by NADH-ubiquinone reductase, which transfers electrons from a flavin complex to several iron-sulfur (Fe-S) centers and then to coenzyme Q10 (ubiquinone Q). Each of these steps in electron transport within complex I has been suggested to play a role in $\mathrm{O}_{2}^{\circ-}$ generation; thus, the flavin mononucleotide site, $\mathrm{Fe}-\mathrm{S}$ clusters, and the complex I-associated ubiquinones may all contribute to electron leak $(149,172,196,227,229,233)$. Blocks in respiration (downstream of complex I) can lead to excess reduction of the electron carriers in complex I, causing electron leak, and enhanced production of $\mathrm{O}_{2}^{\bullet-}$. Although complex II (succinate dehydrogenase) does not generate $\mathrm{O}_{2}^{\bullet-}$, reverse flow of electrons from complex II to complex I may

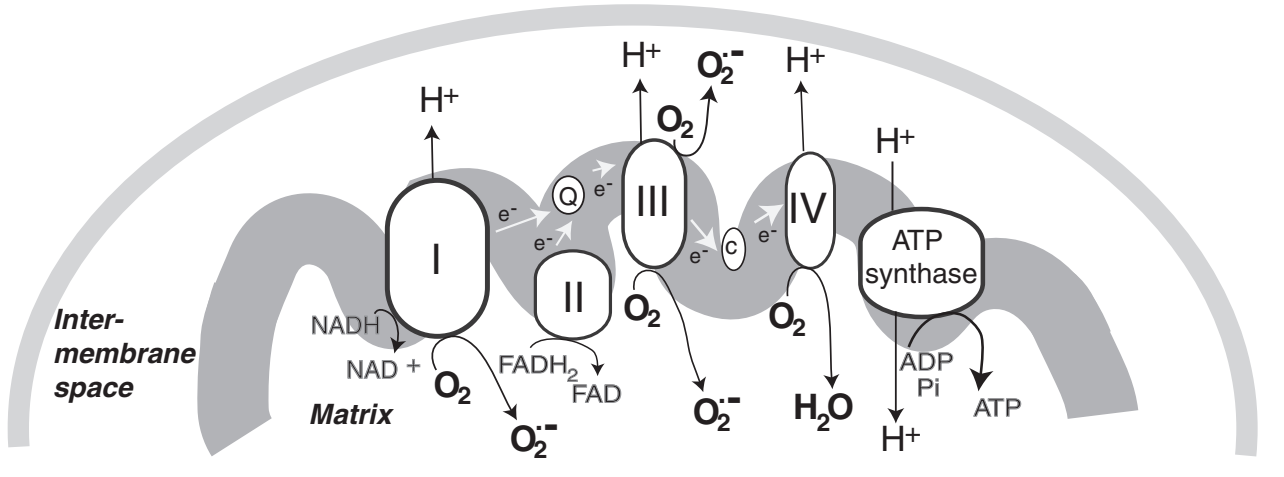

FIG. 1. Mitochondrial sites of superoxide production. Mitochondrial electron transport complexes (I-IV) are located on the inner mitochondrial membrane. The electron transport in mitochondria starts with the extraction of electrons from NADH (complex I) or $\mathrm{FADH}_{2}$ (complex II) generated in the tricarboxylic acid (TCA) cycle. Electrons are transferred along the path shown in the figure, resulting in the reduction of $\mathrm{O}_{2}$ to water at complex IV. During this process, protons $\left(\mathrm{H}^{+}\right)$are pumped by complexes I, III, and IV into the intermembrane space to form an electrochemical gradient $\left(\mathrm{H}^{+}\right.$potential or $\left.\Delta \psi\right)$ at the inner mitochondrial membrane. ATP synthase utilizes the stored energy of this proton gradient to drive the formation of ATP (proton motive force) from ADP and inorganic phosphate (Pi). Electron leak during respiration leads to the formation of superoxide anion $\left(\mathrm{O}_{2}^{\bullet-}\right)$ due to the incomplete reduction of $\mathrm{O}_{2}$ at complex I and complex III (223, 349). $\mathrm{O}_{2}^{\bullet-}$ formed at complex I is released into the mitochondrial matrix, whereas $\mathrm{O}_{2}^{\bullet-}$ formed at complex III can be released either to the matrix or to the intermembrane space, depending on the disposition of complex III from which it forms. 
contribute to electron leak from complex I under some experimental conditions, such as when cells or isolated mitochondria depend on succinate as a source of electrons $(70,262)$. It is unclear how much of a role reverse electron flow plays in normal in vivo respiration $(303,379)$; however, in studies of isolated mitochondria, this mechanism is capable of generating the highest rates of $\mathrm{O}_{2}^{--}$production. The role of electron transport in $\mathrm{O}_{2}^{--}$generation is reviewed further in $(304,403)$.

As discussed throughout this review, post-translational modification of mitochondrial subunits, such as complex I, can either promote or attenuate the generation of $\mathrm{O}_{2}^{\bullet-}$. Thus, thiol oxidation of complex I can promote $\mathrm{O}_{2}^{\bullet-}$ generation, whereas S-nitrosation of complex I suppresses complex I activity to reduce $\mathrm{O}_{2}^{\bullet-}$ leak (98) (see sections II.D.3 and III.A3.). S-glutathiolation, which may be enhanced under oxidant stress, has been shown to preserve complex I activity and decrease $\mathrm{O}_{2}^{\bullet-}$ production under some conditions and inhibit complex I activity to enhance $\mathrm{O}_{2}^{\bullet-}$ production under other conditions (416) (see section II.D.2). Other ETC subunits may similarly be modified by redox mechanisms to modulate electron transport and modify $\mathrm{O}_{2}^{\bullet-}$ production in mitochondria. Complex I and II acetylation, which are regulated by $\mathrm{NAD}^{+}$-dependent deacetylases or sirtuins (SIRT) localized to mitochondria, can also modulate the activities of complex I and II (see section II.E.2) to alter respiration and ROS production (see Table 1 for a summary of these targets).

\section{Redox and antioxidant systems}

Owing to the necessity of keeping ROS within a range compatible with normal cellular function, organisms have evolved a number of modulating antioxidant mechanisms. As mentioned in the previous section, the SOD family catalyzes the conversion of $\mathrm{O}_{2}^{\bullet-}$ to hydrogen peroxide. The members of this family are located in distinct cellular and extracellular compartments to target locally produced $\mathrm{O}_{2}^{\bullet-}$. Once formed, hydrogen peroxide is enzymatically reduced by catalase or members of the glutathione peroxidase (GPx) and

Table 1. Redox Modifications That May Influence Mitochondrial Function Directly or Indirectly

\begin{tabular}{|c|c|c|}
\hline Biological function and target protein(s) & Redox modification & Consequence/mitochondrial effect \\
\hline \multicolumn{3}{|l|}{ Energetics } \\
\hline \multirow[t]{2}{*}{ Complex I } & S-SG & $\begin{array}{l}\text { Either increases activity/decreases } \mathrm{O}_{2}^{\bullet-} \text {, } \\
\quad \text { or decreases activity/increases } \mathrm{O}_{2}^{\bullet-}\end{array}$ \\
\hline & S-NO; acetylation & Decreases activity/increases or decreases $\mathrm{O}_{2}^{\bullet-}$ \\
\hline Complex II & Acetylation & Inhibitory \\
\hline Complex IV & Cys-oxidation/S-NO & Inhibitory \\
\hline ATPase & S-NO & Inhibitory \\
\hline \multicolumn{3}{|l|}{ TCA enzymes } \\
\hline Aconitase / $\alpha-\mathrm{KGDH}$ & S-SG/Cys-oxidation & Inhibitory \\
\hline ICDH & Acetylation & Inhibitory \\
\hline $\mathrm{MDH}$ & Acetylation & Activating \\
\hline \multicolumn{3}{|l|}{ Mitochondrial transporters } \\
\hline ANT & S-S; S-NO & Modulates ADP transport/MPT and cell death \\
\hline VDAC & Cys oxidation & Possible role in apoptosis \\
\hline $\mathrm{UCP}$ & S-SG & Maintains inactive state \\
\hline \multicolumn{3}{|l|}{ Antioxidant enzymes } \\
\hline GPx-1 & Se-oxidation & Loss of Se, inhibitory \\
\hline & Se-NO & Inhibitory \\
\hline Grx1 & $\mathrm{S}-\mathrm{S} ; \mathrm{Cys}-\mathrm{NO}$ & Inhibitory \\
\hline Grx2 & Oxidation & Loss of $[\mathrm{Fe}-\mathrm{S}]$ group releases active monomers \\
\hline GR & $\mathrm{S}-\mathrm{NO} ; \mathrm{S}-\mathrm{OH} ; \mathrm{S}-\mathrm{O}_{2} \mathrm{H}$ & Inhibitory \\
\hline $\operatorname{Tr} x$ & S-S & Releases ASK1 to activate JNK-mediated apoptosis \\
\hline Prx & $\begin{array}{l}\text { Cys-oxidation (S-S; S-OH; } \\
\left.\quad \mathrm{S}-\mathrm{O}_{2} \mathrm{H} ; \mathrm{S}^{-} \mathrm{O}_{3} \mathrm{H}\right)\end{array}$ & Inhibitory \\
\hline \multicolumn{3}{|l|}{ Mitochondrial networks } \\
\hline Drp1 & $\mathrm{S}-\mathrm{NO}$ & Possibly stimulates mitochondrial fission \\
\hline \multicolumn{3}{|l|}{ Mitochondrial biogenesis } \\
\hline$P G C-1 \alpha$ & Acetylation & Inhibitory \\
\hline \multicolumn{3}{|l|}{ Apoptosis/autophagy } \\
\hline Caspase 3 & $\mathrm{~S}-\mathrm{NO}$ & Inhibitory; attenuates apoptotic signaling \\
\hline BCL-2 & S-NO & $\begin{array}{l}\text { Decreases proteasomal degradation of BCL-2; } \\
\text { attenuates apoptosis }\end{array}$ \\
\hline ATG4 & Cys-oxidation & Inhibits protease activity to promote autophagy \\
\hline L-type $\mathrm{Ca}^{2+}$ channel & Cys-oxidation & Inhibitory, attenuates $\mathrm{Ca}^{2+}$-mediated apoptosis \\
\hline
\end{tabular}

ANT, adenine nucleotide transporter; ASK1, apoptosis signal-regulated kinase 1; Cys, cysteine; Drp1, dynamin-related protein-1; Fe-S, iron-sulfur; GPx, glutathione peroxidase; GR, glutathione reductase; Grx, glutaredoxin; ICDH, isocitrate dehydrogenase; JNK, c-jun Nterminal kinase; $\mathrm{MDH}$, malate dehydrogenase; MPT, mitochondrial permeability transition; $\mathrm{O}_{2}^{\boldsymbol{0}^{-}}$, superoxide anion; PGC-1, peroxisome proliferator-activated receptor- $\gamma$ coactivator-1; Prx, peroxiredoxin; TCA, tricarboxylic acid; Trx, thioredoxin; UCP, uncoupling proteins; VDAC, voltage-dependent anion channel; $\alpha-\mathrm{KGDH}, \alpha$-ketoglutarate dehydrogenase. 
peroxiredoxin (Prx) families. The relative effect of each of these types of enzymes on the removal of hydrogen peroxide in a particular cell type may depend on their expression levels, the source and concentration of hydrogen peroxide, their compartmental localization, and other redox-active components that modify their function. Figure 2 illustrates how the antioxidant systems in the mitochondrion contribute to its regulation of ROS release. All mitochondrial antioxidant enzymes are encoded by nuclear chromosomal genes and subsequently imported into the mitochondria after their production in the endoplasmic reticulum. Mitochondrial function, however, is affected by redox-dependent processes that occur throughout the cell, and, possibly, by the extracellular redox state as well $(155,184)$, as discussed further below. For these reasons, in this section we next discuss the complement of antioxidant enzymes that modulate cellular ROS flux, highlighting the redox-dependent processes that modulate their function.

1. SODs. SOD1 or the copper/zinc-dependent SOD $(\mathrm{Cu} / \mathrm{ZnSOD})$ is an intracellular enzyme, widely distributed

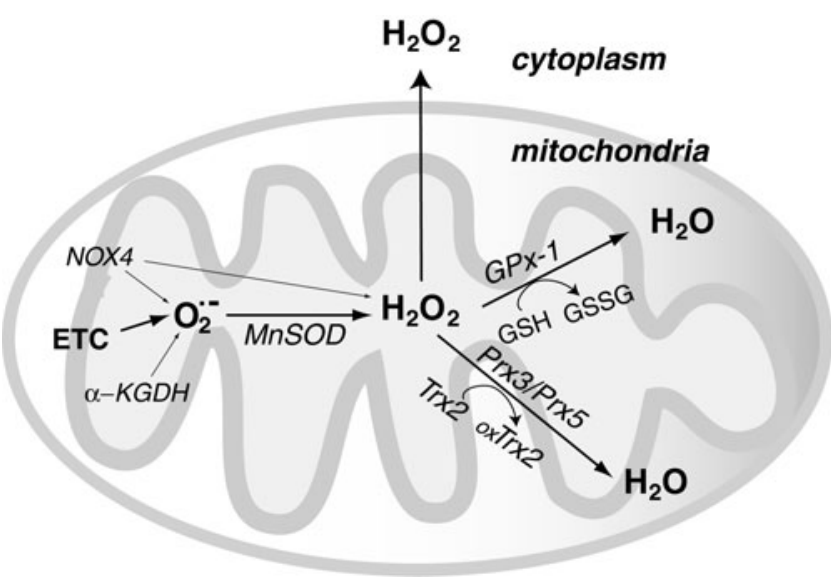

FIG. 2. Mitochondrial hydrogen peroxide $\left(\mathrm{H}_{2} \mathrm{O}_{2}\right)$ flux. Most of the reactive oxygen species (ROS) generated in mitochondria is in the form of $\mathrm{O}_{2}^{--}$from the electron transport chain (ETC) $(223,349)$. Recent evidence suggests that NADPH-dependent oxidase 4 (NOX4) may be localized to mitochondria, where it may produce hydrogen peroxide and/or $\mathrm{O}_{2}^{--}$(118). The TCA cycle enzyme $\alpha$-ketoglutarate dehydrogenase $(\alpha-\mathrm{KGDH})$ is also a potential source of $\mathrm{O}_{2}^{\bullet-}$ (423). Within the mitochondrial matrix, manganesedependent superoxide dismutase (MnSOD) is the only SOD present (236); it reduces $\mathrm{O}_{2}^{--}$to hydrogen peroxide. Mitochondrially located glutathione peroxidase-1 (GPx-1) utilizes glutathione (reduced glutathione [GSH]) as a cosubstrate in the reduction of hydrogen peroxide to water (126, 133). Similarly, the mitochondrially targeted peroxiredoxins (Prx3 or Prx5) reduce hydrogen peroxide using thioredoxin $2(\operatorname{Tr} x 2)$ to regenerate the active site $(66,73)$. Also important are glutathione reductase (GR) and Trx reductase (TrxR), which serve to reduce oxidized GSH and Trx, respectively (not represented in this figure). In the intermembrane space, it is thought that copper/zinc-dependent SOD $(\mathrm{Cu} / \mathrm{ZnSOD})$ may play a role in reducing $\mathrm{O}_{2}^{\circ-}$ that is released from complex III. Within this space and in the cytosol, GPx-1 and Prx family members reduce hydrogen peroxide produced and released from the mitochondria or other sources. throughout the cell cytoplasm, nucleus, and microsomes; it has even been detected in the mitochondrial intermembrane space of rat liver mitochondria (318). Missense mutations of the SOD1 coding region are associated with some familial cases of amyotrophic lateral sclerosis (ALS), suggesting a role for oxidative stress in this disorder (27). It is not yet resolved, however, as to how these genetic alterations contribute to this degenerative disease, as SOD1-deficient mice are viable with no clear ALS phenotype. Other findings suggest that ALSassociated mutant forms of SOD1 aggregate in the cytoplasm and intermembrane space of the mitochondria and suggest that this aggregation contributes to cell death (75). Recent findings implicate redox mechanisms in the toxicity of these aggregating mutant SOD1 enzymes, as overexpression of the mitochondrial glutaredoxin 2 (Grx2), a redox-active protein that catalyzes disulfide exchange reactions and the reduction of protein-mixed disulfides (177), preserves mitochondrial metabolism and prevents cell death caused by coexpressed mutant SOD1 variants, at least in cell culture models (130). Although SOD1 deficiency in a knockout mouse may not cause ALS symptoms, these mice do exhibit oxidative stress in various tissues $(39,190,442)$. Some of the redox changes caused by SOD1 deficiency may be secondary to oxidative inactivation of glutathione peroxidase 1 (GPx-1), a key intracellular enzyme that reduces hydrogen peroxide to water. As discussed further below, GPx-1 plays an essential role in modulating intracellular hydrogen peroxide with consequences for growth factor-mediated signaling and mitochondrial ATP production (169). The extracellular SOD (ecSOD) is a secreted glycoprotein that can associate with cell surface glycosaminoglycans to mitigate the effects of extracellular $\mathrm{O}_{2}^{\bullet-}$. This extracellular enzyme plays a crucial role in preserving vascular function by attenuating the inactivation of endothelial $\mathrm{NO}^{\bullet}$ by $\mathrm{O}_{2}^{\bullet-}$, thereby preserving endotheliumdependent vasodilatory responses of vascular smooth muscle cells, while also limiting oxidative damage caused by the formation of peroxynitrite. In ecSOD knockout mice, lack of ecSOD has no significant effect on blood pressure under normal physiological conditions; however, lack of ecSOD augments angiotensin II (AII)-induced hypertension and promotes endothelial dysfunction. Endothelial dysfunction, characterized by a lack of dilation of blood vessels in response to endothelium-dependent vasoactive substances, such as acetylcholine, is consistent with a deficiency of bioavailable $\mathrm{NO}^{\bullet}(158,200,266)$. Recent findings indicate that extracellular alterations in redox potential promote alterations in intracellular redox balance that affect mitochondrial production of ROS $(155,184)$. Based on this concept, alterations in the levels of ecSOD or other extracellular antioxidants could theoretically alter cell function by influencing intracellular redox status.

As the only mitochondrial matrix SOD, manganesedependent SOD (MnSOD) has a crucial role in the inactivation of mitochondrial (matrix) $\mathrm{O}_{2}^{\bullet-}$. Its deficiency causes early neonatal death in knockout mice, which suffer from dilated cardiomyopathy and neurodegeneration $(236,252)$. Furthermore, SOD2 expression is induced by a number of stimuli, including cytokines such as tumor necrosis factor- $\alpha$ (TNF- $\alpha$ ) (453), hormones such as leptin (478), and pharmacological treatments such as resveratrol (144). SOD2 transcription is also upregulated as part of the peroxisome proliferatoractivated receptor- $\gamma$ coactivator- 1 alpha (PGC- $1 \alpha$ )-mediated 
biogenic response that increases mitochondrial biosynthesis along with antioxidant enzymes that modulate oxidant flux (398).

2. Catalase and NADPH. Catalase is a heme-containing tetramer that reduces hydrogen peroxide to water. Although NADPH is not a true cofactor, as the catalytic reduction of hydrogen peroxide by catalase does not require NADPH, NADPH binds to each monomeric subunit of catalase and provides electrons to prevent catalase inactivation by hydrogen peroxide (216); in addition, NADPH binding facilitates the assembly of catalase tetramers, stabilizing the molecule and optimizing its activity (166). Thus, maintenance of the NADPH pool is essential for preserving catalase activity during oxidant stress. Although catalase is highly efficient at reducing hydrogen peroxide, it may not play a central role in modulating hydrogen peroxide responses following many stimuli as it is localized mainly in peroxisomes. Rat liver and heart mitochondria, however, depend, in part, on catalase to reduce endogenous or exogenous hydrogen peroxide (339, 369). These and other findings suggest that in some tissues and cells, catalase is important in maintaining overall redox homeostasis. In particular, in erythrocytes, catalase, together with GPx-1, significantly contributes to the detoxification of hydrogen peroxide (146) as acatalasemic erythrocytes are less able to detoxify hydrogen peroxide. Interestingly, more recent studies with Prx1- and Prx2-deficient mice suggest that these antioxidant enzymes may also significantly contribute to antioxidant protection of erythrocytes.

3. GPx, reduced glutathione, Grxs, and glutathione reductase. GPxs are a family of selenocysteine (Sec)-containing enzymes (with one exception, Gpx-5). GPx-1, the first-identified of these, was initially found in erythrocytes (298). GPx-1 and some of the other members of this family rely on reduced glutathione (GSH) as a cofactor in the reductive inactivation of hydrogen peroxide and/or lipid hydroperoxides (Fig. 3). (Other members of the GPx family rely on other thiol reductants, such as thioredoxin [Trx], which is also used as a source of reducing equivalents by many Prx family members.) During the reduction of peroxide targets, the GSH electron donor is oxidized to oxidized glutathione (GSSG), which is then recycled by glutathione reductase (GR) and relies on NADPH for its reducing activity $(358,359)$. Thus, the maintenance of GSH for optimal GPx enzyme activity is dependent on maintaining the pool of NADPH stores.

GPx-1 is the most widely studied member of this family [reviewed in Ref. (265)]. In mammalian cells, it is a selenoprotein with Sec at its active site; it is ubiquitously expressed, found in cytoplasm and mitochondrial compartments; and its deficiency is associated with oxidative stress. GPx-1-deficient mice manifest endothelial dysfunction, characterized by a lack of normal responses to endothelium-dependent vasoactive compounds $(134,135)$. GPx-1 was also found to play a role in ischemia-reperfusion injury in cardiac and neuronal models, and atherosclerosis in susceptible mouse models $(103,248,256,418,452,459)$. In human subjects, lower levels of GPx-1 activity are associated with atherogenesis and an increased risk for cardiovascular events $(42,377)$. Furthermore, GPx-1 modulates mitochondrial function as its overexpression decreases mitochondrial electrochemical potential (decreases $\Delta \Psi$ ), ROS generation, and ATP synthesis (169).
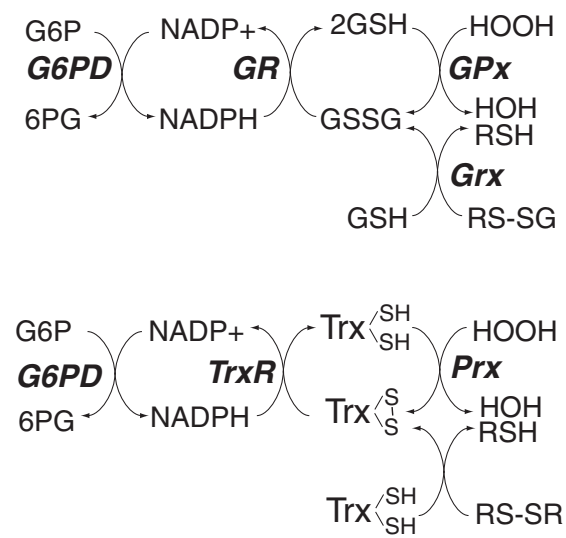

FIG. 3. Role of NADPH and glucose-6-phosphate dehydrogenase (G6PD) in redox homeostasis. Cellular stores of NADPH are essential for the reductive recycling of GSH and Trx, as it is a necessary cofactor for the enzymes GR and TrxR, which restore the reduced pools of these redox-agents, respectively. [Through different mechanisms involving enzyme stability, NADPH is also essential for the activity of catalase $(166,216)]$. As illustrated in this diagram, GSH is necessary for both the activity of GPxs, which reduce hydrogen and lipid peroxides (133), and glutaredoxin (Grx), which reduces protein-mixed disulfides with GSH (RS-SG) (209). To some extent, Grx may also reduce protein disulfides as well (not in the diagram), similar to the actions of Trx, which plays an important role in modulating protein disulfides, including those of peroxiredoxins (Prx). Trx is, thus, essential for Prx-mediated hydrogen peroxide reduction. In mitochondria, GPx-1 and GPx-4 modulate the reduction of hydrogen and lipid peroxides. Mitochondrially localized GR and Grx2, as well as their cytoplasmic forms, contribute to overall GSH/oxidized glutathione (GSSG) redox status. Prx3 and Prx5, along with Trx2 and TrxR2, comprise the mitochondrial Trx system. Extracellular and cytosolic redox potential may also influence the redox status of these antioxidant proteins.

Consistent with a role for GPx-1 in modulating mitochondrial oxidants, its deficiency in mice augments release of oxidants from respiring mitochondria (126).

All but one of the members of the mammalian GPx (GPx1 through GPx-6) family are selenoproteins, with the amino acid Sec. GPx-5, which has a cysteine (Cys) substituted for the active site $\mathrm{Sec}$, is only expressed in the epididymis, and appears to be important in providing antioxidant protection to spermatozoa (90). GPx-2 is highly similar to GPx-1 except that its expression is limited to epithelial cells, especially those of the intestinal tract, where it may play an essential role in preventing tumorigenesis (29). GPx-6 is also limited in its expression to adult olfactory epithelium (344), but has not been widely studied. GPx-3 is a secreted glycoprotein, and its in vivo cofactor is a subject of debate. Deficiency of GPx-3 in humans or knockout mice potentiates platelet activation and thrombosis (139, 193, 212). Furthermore, in GPx-3 knockout mice, deficiency of this extracellular antioxidant causes endothelial dysfunction and increased sensitivity to neuronal injury in a brain model of ischemia reperfusion injury by mechanisms related to its key functions as an antioxidant enzyme and regulator of plateletdependent thrombosis (193). 
GPx-4 is unique in that, unlike the other GPx family members, it exists as a monomer and, intracellularly, it primarily targets membrane lipid hydroperoxides. In addition, unlike the other GPx-family members, GPx-4 knockout is lethal in mice (465). GPx-4 is expressed as multiple isoforms generated from the same gene: one of these targets the mitochondria where it has a vital role in protecting against membrane lipid peroxidation; in its absence, mitochondria accumulate damaging lipid peroxides, leading to apoptogen release $(253,383)$. Targeted overexpression of the mitochondrial form of GPx-4 in transgenic mice attenuates cardiac ischemia-reperfusion injury, lowers mitochondrial lipid peroxidation, and preserves ETC complex activities after ischemia-reperfusion injury, confirming a vital role of redox alterations in modulating mitochondrial function (106).

Importantly, the Sec functionality in the mammalian GPxs is a redox-active site $(\mathrm{Se}-\mathrm{H})$, similar to Cys $(\mathrm{S}-\mathrm{H})$. During the enzymatic reduction of hydrogen peroxide, Sec undergoes sequential oxidation by hydrogen peroxide to form a selenenic acid, Se-OH, followed by reaction with GSH to form a Se-SG intermediate before a second GSH combines to form GSSG, resolving the active site to Se-H $(133,278)$. Upon exposure to oxidant stress, there is accordingly an increase in the oxidation of GSH and a concomitant decrease in the intracellular ratio of GSH/GSSG that appears to be related to the action of GPx-1 as in the absence of GPx- 1 there is less detectable change in GSSG $(140,254)$. Interestingly, the cellular pool of GSH is also necessary to prevent oxidative inactivation of GPx-1 by excess oxidants (433). In vitro, GPx-1 has been found to be highly susceptible to oxidative inactivation by $\mathrm{O}_{2}^{\bullet-}$, especially in the absence of GSH $(85,331)$. The redoxactive Sec site may also be a target for other free radicalmediated reactions, forming Se-NO in the presence of $\mathrm{NO}^{\bullet}$ $(19,20)$. Isolated older erythrocytes have been found to accumulate inactivated GPx-1, suggesting that oxidative inactivation of GPx-1 occurs in vivo possibly due to insufficient NADPH and GSH stores in older erythrocytes. The process of GPx-1 inactivation is irreversible through the loss of Se-H by beta-elimination of oxidized $\mathrm{Se}$ as $\mathrm{H}_{2} \mathrm{SeO}_{2}$, resulting in the formation of dehydroalanine. Dehydroalanine, in turn, can be detected by mass spectrometry or through its interaction with cysteamine, which can be linked to biotin for detection and quantification (85). Other reports indicate that excess oxidants in vivo may similarly result in Sec loss from GPx-1, as SOD1deficient mice have a substantial loss of GPx-1 activity that corresponds with an increase in detectable dehydroalanine in GPx-1 (442). This is one example of the auto-amplification of ROS generation (ROS-mediated ROS generation), with increased ROS promoting more ROS generation, as loss of an enzyme that reduces $\mathrm{O}_{2}^{\bullet-}$ causes inactivation of an enzyme that can eliminate hydrogen peroxide. Other instances of ROS-mediated ROS generation and its consequences for mitochondrial function are discussed further below.

Intracellularly, glutathione is predominantly reduced (GSH), with the cytosolic content of GSH maintained via the cyclic reduction of GSSG by GR, de novo biosynthesis, and cellular uptake of extracellular glutathione (disulfide) (288). Accurate measurements of GSSG are difficult, especially in isolated intracellular compartments, as GSH is easily oxidized to GSSG during sample isolation. Nonetheless, GSH has a low redox potential in cytosol $\left(E_{0}=-260\right.$ to $\left.-200 \mathrm{mV}\right)(374)$ and measurements from mitochondria suggest an even lower glu- tathione redox potential $(-330$ to $-300 \mathrm{mV})$ in this intracellular compartment (156), consistent with ratios of GSH:GSSG of over 100:1 (374). This ratio is in contrast to the endoplasmic reticulum where GSH:GSSG ratios between 1:1 to 1:3 (and corresponding redox state of $-180 \mathrm{mV}$ ) have been reported $(182,374)$.

Recent studies have utilized redox-sensitive biosensors to monitor real-time measurements of intracellular GSH-redox state. In yeast cells, this has been accomplished by using a redox-sensitive yellow fluorescent protein (rxYFP) that forms disulfide bonds in proportion to intracellular GSH redox potential (319). Using a series of genetically deficient yeast lines, it was shown that equilibration of the sensor with intracellular GSH:GSSG relies on intracellular Grx. Grx proteins are small (12-16 kDa) redox active proteins that catalyze thiol-disulfide exchange reactions of oxidized protein disulfides and mixed disulfides, as well as those of low-molecular-weight disulfides such as GSSG (205) [Grx are discussed further below]. Other investigators have further modified the rxYFP protein to target it to mitochondrial compartments (178). Despite the advantage of the real-time values that can be tracked by these intracellular sensors, there may be other drawbacks of these protein-based probes due, in part, on their reliance on cellular Grxs and their lag-time to equilibrate with cellular GSSG (178, 319). In addition, these redox probes are highly sensitive to changes in intracellular $\mathrm{pH}$. Furthermore, it is unclear whether other intracellular components can alter their redox status, although, to date, no other intracellular redox component has been found to influence their function $(178,319)$. A similar biodetector, engineered by combining human Grx with a redox-sensitive GFP, was used to image GSSG-redox changes within human cells (163). This recombinant sensor apparently equilibrates more quickly with cellular GSSG than redoxsensitive probes that lack the Grx domain in cis with the redox-sensor domain. Overall, relative measurements calculated with these biosensors suggest even lower redox potentials for intracellular GSH $(-289 \mathrm{mV})$ and higher GSH:GSSH ratios than those calculated by measuring GSH and GSSG concentrations with biochemical methods (163, $178,319)$. These biosensors hold great promise in furthering our understanding of GSH redox status in normal cellular processes and those associated with redox stress.

It has been estimated that mitochondria may contain $\sim 10 \%-15 \%$ of cellular GSH, but according to a recent review on mitochondrial GSH, the overall concentration of GSH is similar in cytosolic and mitochondrial compartments (measured between 1 and $14 \mathrm{mM}$ ) (275). In mitochondria, GSH stores are distinct from that in the cytosol, and maintained by active transport through the dicarboxylate or 2-oxoglutarate carriers $(287,393)$ and by enzyme-mediated reduction of GSSG by mitochondrially targeted GR (414). Deficiency of cellular glutathione leads to mitochondrial damage and apoptosis (287), in part, by loss of GPx-mediated protection against mitochondrial oxidants. GSH, however, has other cellular functions, including the formation of mixed disulfides with redox-active protein thiols. These interactions can modulate the reactivity and activity of a variety of cellular targets, as discussed further below.

Glutaredoxins (Grx) are important determinants of proteinmixed disulfides. In yeast, five isoforms of Grx have been described, whereas in mammalian cells there are three isoforms, Grx1, Grx2, and Grx5. These proteins have some conserved features, including a Trx fold. Grx1 is a cytosolic 
protein with a Cys-Pro-Tyr-Cys active site motif. Alternative splicing of the Grx2 transcript results in the production of one enzyme isoform that targets to the nucleus and one that is found in the mitochondrial matrix (267). Grx2 contains a different active site motif, Cys-Ser-Tyr-Cys, and reduction of the oxidized form of Grx 2 can be mediated by GSH or NADH and thioredoxin reductase (TrxR) (195). Grx1 and Grx2 catalyze protein disulfide reduction (R-S-S-R) via a dithiol mechanism and protein-mixed disulfide (R-S-S-G) reduction via a monothiol mechanism (177). Notably, Grx2 added to isolated mitochondrial membranes mediates the reversible glutathiolation of proteins in a manner dependent on the ratio of GSH/ GSSG (36). One of the targets of glutathiolation, mitochondrial complex I, was also found to have decreased activity under oxidative conditions favoring glutathiolation, suggesting an important role for Grx2 in modulating mitochondrial ROS production by reducing complex I glutathiolation. Grx2 also differs from Grx1 in that it is associated with $2 \mathrm{Fe}-2 \mathrm{~S}$ clusters (194). The Grx2/Fe-S complex, which has mostly been found in mitochondria, consists of 2 Grx2 linked to the Fe-S cluster through the N-terminal Cys of the Grx2 active site. Two GSH molecules were also found to associate with this Grx2/Fe-S complex, which has been suggested to function as a redox sensor. Thus, Fe-S sequesters active sites of Grx2 in the setting of high GSH:GSSG ratios and releases active monomers following oxidative shifts in the redox state (194). Grx2 is essential for the normal cellular responses to oxidant stress. In cultured human cells, depletion of mitochondrial Grx2 was found to increase cell sensitivity to apoptosis in response to agents causing oxidant stress (255). Other studies have found that overexpression of Grx2 decreased apoptosis in response to similar agents, confirming a role for Grx2 in protecting against redox-mediated apoptosis (124). Neither of these studies on the role of Grx2 in apoptosis reported changes in protein glutathiolation; however, the latter study, which focused on apoptotic signaling, found that Grx overexpression reduced cardiolipin oxidation to prevent cytochrome c release and caspase activation. Grx5 also protects against oxidative stress and apoptosis; Grx5 overexpression attenuates hydrogen peroxide-induced apoptosis and cardiolipin oxidation in osteoblasts, whereas its deficiency augments cell death (259). Similar to Grx2, the eukaryotic Grx5 is also primarily found in mitochondria. Unlike Grx1 and Grx2, Grx5 has only a single thiol in its active site: Cys-Gly-Phe-Ser (in human Grx5) (205). Grx5 has also been found to play an important role in Fe-S cluster formation in yeast, as well as in higher eukaryotic organisms $(355,436)$. Thus, a deficiency of Grx5 has been found to alter cellular iron metabolism, the production of heme, and the activity of Fe-S cluster-dependent enzymes, such as aconitase $(259,299,448)$. The role of Fe-S clusters in modulating the activities of mitochondrial ETC complexes and enzymes of the tricarboxylic acid (TCA) cycle is discussed further in section VI.

GR is essential for the recycling of GSSG to GSH, thereby playing an important role in maintaining Grx and GPx enzyme activities. GR enters the mitochondria via an N-terminal targeting sequence but is also found in the cytosol (186). Its activity has been shown to regulate Akt and endothelial NOS (eNOS) phosphorylation in response to flow in bovine aortic endothelial cells, in part, by maintaining Grx activity (441). Interestingly, the function of GR can be oxidatively inactivated at a Cys residue in the active site $(372,428)$. In studies of renal nephrotoxicity, oxidative inactivation of GR and GPx by alkylating agents was found to contribute to oxidative stress, characterized by increased lipid peroxidation and cell death in renal proximal tubules that involve mitochondrial disruption and the production of excess oxidants by mitochondria (428). In RAW264.7 cells, activation by a mixture of endotoxin and interferon- $\gamma$, a condition that increases endogenous NO production through the stimulation of inducible NOS (iNOS) in this macrophage-like cell line, results in S-nitrosation of GR. Loss of GR function was also correlated with decreased cellular GSH and increased oxidant stress in these cells (59). Other studies similarly suggest that conditions that inhibit GR as well as Grx disrupt the cellular GSH:GSSG redox state and contribute to cell death; for example, suppression of GR and Grx appears to be responsible for oxidative damage caused by exposure of human monocyte-derived macrophages to oxidized low-density lipoprotein (443). Mitochondrially localized GR has also been shown to be instrumental in maintaining cellular growth and the activities of some Fe-S proteins, at least in fission yeast Schizosaccharomyces pombe (395); at the current time, it is unclear whether GR in higher eukaryotes similarly influences the function of Fe-S containing proteins.

4. Prxs, Trx, and TrxR. First identified in yeast, the mammalian peroxiredoxins (Prx) comprise a large family of enzymes that also catalyze the reductive metabolism of hydrogen peroxide or lipid peroxides (346). Most peroxiredoxins utilize Trx as an electron donor (Fig. 3), although Prx6 apparently uses GSH (132). These enzymes have been subgrouped according to the number of Cys involved in their enzymatic action: thus, Prx1-4 are typical 2-Cys forms, Prx 5 is an atypical 2-Cys form, and Prx6 is a 1-Cys enzyme. As homodimers, the typical 2-Cys Prxs reduce hydrogen peroxide by sequentially forming a sulfenic acid at one Cys residue that then forms an intersubunit disulfide, which is resolved by Trx (66). In the atypical 2-Cys Prx5, which is a monomer, the disulfide (from vicinal dithiols) forms intramolecularly after sulfenic acid formation. The Prx6 mechanism is similar to that of the GPxs where an S-OH (sulfenic acid) formed at the active site is reduced by GSH after the formation of an intermediate mixed disulfide (S-SG). Recent findings suggest a necessary role for glutathione-S-transferase $\pi$ in the glutathiolation of Prx6, as Prx6 function is attenuated in the absence and stimulated by the presence of this glutathione-S-transferase (273). Prx6 is also unique in that it can reduce phospholipid hydroperoxides similar to GPx-4 (132); however, of these two phospholipids peroxidases, only GPx-4 targets to mitochondria.

Theoretically, Prx are less efficient at catalyzing hydrogen peroxide reduction than catalase or GPx-1; however, some of the Prx isoforms (Prx1 and Prx2) are highly abundant, and Prxs have a comparatively low $K_{\mathrm{m}}(<20 \mu M)$ for hydrogen peroxide (65). Thus, it seems likely that Prxs regulate normal hydrogen peroxide flux within cells. Experimental evidence supports this concept, as deficiency of various Prxs augments cellular responses to oxidant-inducing stimuli, whereas their overexpression enhances antioxidant protection. Thus, cells isolated from mice with deficiencies in Prx1 or Prx2 show increased production of hydrogen peroxide and increased sensitivity to oxidant damage $(88,239,313)$. In particular, erythrocytes from either Prx1- or Prx2-deficient mice have 
decreased lifespan and excess accumulation of oxidative proteins. Similarly, deficiency of the mitochondrially specific Prx3 in cells increases mitochondrial production of ROS and promotes apoptotic changes in mitochondria, whereas its overexpression decreases ROS and attenuates apoptotic signaling (73). Furthermore, Prx6-deficient mice have increased cardiac ischemia-reperfusion injury with an increase in oxidative stress (308).

Thus, as shown in many studies, Prxs protect against the damaging consequences of excess oxidants in cell culture and in vivo systems; however, many of the mammalian 2-Cys forms are susceptible to overoxidation, a process in which the $-\mathrm{S}-\mathrm{OH}$ of the oxidized Cys-active site is further oxidized to $-\mathrm{SO}_{2} \mathrm{H}$ (sulfinic acid) $(338,462)$. As discussed further in section II.D.1, in most proteins, sulfinic acid formation is an irreversible oxidation state. The intriguing aspect of sulfinic acid formation in Prxs is the existence of an enzyme, sulfiredoxin (Srx) (74), that specifically targets and reduces sulfinic acid in 2-Cys Prxs. Srx is only found in eukaryotes, suggesting that it evolved as a compensatory mechanism to restore function of eukaryotic 2-Cys Prxs, which, unlike their prokaryotic counterparts, are highly sensitive to overoxidation. The importance of this overoxidation/reduction mechanism is uncertain at this time. Recent work using an Srx1-deficient mouse indicates that Srx protects mice from lipopolysaccharide (LPS)-induced sepsis as deficient mice have increased sensitivity to LPS (333), a stimulus known to produce oxidant stress in vivo. Although the in vivo presence of overoxidized Prxs was not shown in this study, in mouse embryonic fibroblasts, Srx deficiency resulted in excess accumulation of intracellular oxidants in untreated cells and increased oxidation of Prx $1 / 2$ in response to exogenous hydrogen peroxide, implicating Srx-mediated preservation of the redox state of Prx $1 / 2$ in the antioxidant mechanisms of Srx.

The floodgate theory suggests that peroxiredoxins act as sensors of hydrogen peroxide concentration such that their oxidative inactivation allows downstream hydrogen peroxide-mediated signaling to proceed (454). Sulfiredoxin restoration of Prxs would be one mechanism of reestablishing the gateway. Many arguments can be made for or against this theory; however, experimental evidence indicates that the multitude of systems regulating hydrogen peroxide flux are not redundant, suggesting that each system (catalase, GPxs and Prxs) may contribute to overall cellular peroxide tone. In models of hydrogen peroxide-dependent hydrogen peroxide flux in Jurkat cells, the relative contributions of GPxs and Prxs to hydrogen peroxide reduction were about 100 -fold greater than that of catalase, with GPx-1 responsible for $44 \%-57 \%$ of the flux and Prxs $32 \%-42 \%$ of the flux (3). Although these findings may not account for all of the molecular interactions involved in cellular hydrogen peroxide flux and may not hold true in all cell types, this model also predicted a significant effect of hydrogen peroxide on the protein disulfide pool. As discussed later, the formation of many disulfide bonds is modulated via the flux of oxidants produced [especially hydrogen peroxide (464)], in part, via mitochondrial respiration $(169,464)$.

The activity of the 2-Cys peroxiredoxins is also regulated by the availability of reduced Trx, a low-molecular-weight redox-active protein (137). Two major forms of Trx, cytosolic (Trx1) and mitochondrial (Trx2), play an important role in reducing protein disulfides, including those of the Prxs. Re- cent findings indicate that mitochondrial $\operatorname{Prx} 3$ can also be functionally reduced by mitochondrial Grx2; this was shown in both in vitro kinetic assays as well as in situ in HeLa cells (170). In HeLa cells, Trx2 deficiency (or Grx2 deficiency) alone had little effect on the Prx3 oxidation; however, simultaneous knockdown of both Trx2 and Grx2 substantially increased Prx3 oxidation. Trx2 is, however, important in maintaining cellular redox status and, similar to the effects of Prx 3 overexpression in attenuating apoptosis, cells with excess Trx2 are more resistant to oxidant-induced apoptosis (79). The mitochondrial (Trx2) and cytosolic (Trx1) forms of Trx have two active Cys residues that are oxidized to form an intramolecular disulfide after the reduction of protein disulfides. Trx-disulfides are reduced by the cytosolic or mitochondrial NADPH-dependent selenoproteins, the TrxR, TrxR1 or TrxR2, respectively. A third TrxR subtype is expressed solely in testes. These proteins are discussed further below. Trx 1 and related family members that are targeted to the cytoplasm and nucleus also modulate the activity of other redox-dependent proteins, including enzymes and transcription factors, to control processes ranging from apoptosis and DNA synthesis to DNA binding by transcription factors. Both Trx1 and Trx2 appear to be essential, as complete deficiency of either subtype causes early embryonic lethality in mice (280, 317). Most of Trx exists in the reduced form in cells and mitochondria, but these proteins can become oxidized under certain apoptotic stresses. Excess oxidation of Trx2 is associated with activation of the mitochondrial transition pore during apoptosis (348), illustrating the importance of Trxredox in maintaining normal mitochondrial function.

The mammalian TrxR proteins are Sec-containing proteins with the Sec amino acid in the pentultimate site of the protein as part of the C-terminal active site (Gly-Cys-Sec-Gly-COOH). Homodimers form the functional TrxR protein in which the monomers are arranged in a head to tail (Ying-Yang) configuration. The mechanisms of TrxR enzymatic reduction of Trx is reviewed in Ref. (18). Interestingly, selenoproteins are essential for modulating both the mammalian Trx system (through TrxRs) and the GSH system (through GPx enzymes). Deficiency of selenium has been associated with cardiomyopathy (Keshan disease) in regions of China with nutritional inadequacy of this micronutrient stemming from its low soil content. Decreased activity of antioxidant enzymes, such as GPx-1 and TrxRs, has been suggested to contribute to the cardiomyopathy associated with selenium deficiency $(82,100$, $145,241)$. Treatment with dietary selenium supplements limits the disease phenotype, consistent with a primary causative role of selenium deficiency in the development of this disease.

Both TrxR1, which is primarily cytosolic, and TrxR2, which is primarily mitochondrial, are essential, as knockout of either gene is embryonically lethal with distinct phenotypes. Loss of TrxR1 causes severe growth retardation and deficits in mesoderm formation, whereas loss of TrxR2 has profound effects on hematopoiesis, liver development, and heart development, with profound anemia and reduced myocardial cell proliferation in TrxR2-deficient embryos $(18,100)$. Conditional knockout solely in cardiac cells implicated TrxR2, but not TrxR1, in heart development. Loss of cardiac-specific TrxR2 was found to result in a dilated cardiomyopathy that caused neonatal death (100). In mouse embryonic fibroblasts with TrxR2 deficiency, there was no evidence of excess ROS by 
RedoxSensor Red CC-1 staining compared to wild-type fibroblasts, suggesting that in the absence of TrxR2, other antioxidant systems could substitute under basal conditions. However, after cellular GSH depletion, these deficient fibroblasts showed enhanced sensitivity to apoptosis compared to wild-type cells and had enhanced ROS production in mitochondria, conditions that could be attenuated by $\mathrm{N}$ acetylcysteine treatments (100). Consistent with the importance of TrxR2 in modulating mitochondrial ROS, this study also found that hearts from the cardiac-specific TrxR knockout mice had severe mitochondrial malformation and mitochondrial swelling (100). Taken together, these findings show the essential nature of the TrxR proteins in development and the importance of mitochondrial antioxidant enzymes such as TrxR2 in cardiac development.

5. Cytosolic and mitochondrial NADPH. NADPH plays an important role in maintaining the activity of all of the hydrogen peroxide-targeting antioxidants (Fig. 3). It is essential to maintain the active state of catalase and is used as a source of reducing equivalents in the enzymatic recycling of the essential cofactors of these enzymes, GSH and Trx. Glucose-6phosphate dehydrogenase (G6PD) is essential for the production and maintenance of the NADPH pool by catalyzing the reduction of $\mathrm{NADP}^{+}$during the first and rate-limiting step of the pentose-phosphate pathway. This enzyme is ubiquitously expressed, and is essential in maintaining cellular redox balance: its deficiency is associated with oxidant stress, whereas its overexpression protects cells against ROS accumulation $(63,244,245)$. In humans, G6PD deficiency, due to the presence of lower activity variants, is common worldwide, but especially in regions with endemic malaria. Deficiency of G6PD actually confers protection against severe forms of malaria, possibly due to the early removal of erythrocytes infected with the malarial parasites owing to their accumulation of oxidative damage (63); however, G6PD deficiency may promote susceptibility to other diseases, such as diabetes and hypertension, owing to redox imbalance and oxidant stress (243). For example, acquired deficiency of G6PD caused by high glucose-mediated inhibition of G6PD expression and activity increases ROS generation in pancreatic islet cells (476). Specifically, increased oxidant stress caused by deficiency of G6PD leads to increased apoptosis, decreased proliferation, and impaired insulin secretion in islet cells. Furthermore, G6PD-deficient mice that contain a hypomorphic G6PD gene (335) have impaired glucose tolerance and smaller islets than wild-type mice (476), further illustrating how G6PD deficiency contributes to diabetes. Curiously, the naturally occurring adrenal steroid hormone dehydroepiandrosterone (DHEA), which is a noncompetitive inhibitor of G6PD, improves insulin sensitivity and obesity in cell systems as well as in human and animal models (461). Although this may be due to the effects of DHEA on enzymes other than G6PD, it is possible that a decrease in NADPHreducing equivalents may promote increased ROS in response to insulin to enhance insulin-mediated signaling and lessen insulin resistance found in obesity or the metabolic syndrome. Other studies find that G6PD deficiency leads to renal oxidant stress, with decreased NADPH and GSH and increased albuminuria (460), and that deficiency of G6PD promotes enhanced cytokine responses to endotoxemic stress with reduced survival (447). Thus, the protective or harmful effect of G6PD suppression may depend on the context: the level of stress, the degree of G6PD inhibition, and whether other systems are simultaneously altered (as mentioned above in the discussion of androgenic steroid inhibition of G6PD). Consistent with a protective role of G6PD, targeted overexpression of G6PD to dopaminergic neurons decreases the toxic effect of 1-methyl-4-phenyl-1,2,3,6 tetrahydropyridine in a mouse Parkinson disease model (289).

Although complete deficiency of G6PD is incompatible with life, it is not the only enzyme that contributes to cellular NADPH biosynthesis. In mitochondria, the enzymatic actions of nicotinamide nucleotide transhydrogenase (NNT), which relies on the mitochondrial electrochemical proton gradient for its activity, regenerates NADPH from $\mathrm{NADP}^{+}$utilizing NADH produced in the TCA cycle (361). Thus, mitochondrial deficiencies that decrease matrix $\mathrm{NADH}$ or mitochondrial membrane potential will theoretically decrease mitochondrial NADPH regeneration, contributing to oxidative stress (226). $\mathrm{NADPH}$ is an essential source of reducing equivalents for GSH- and Trx-dependent antioxidant enzymes, including those in mitochondria. In addition to transhydrogenase, mitochondrial NADPH stores can also be restored by certain dehydrogenases present in the mitochondria, such as isocitrate dehydrogenase (ICDH) and malate dehydrogenase $(\mathrm{MDH})$ (468). $\mathrm{NAD}^{+}$can also be a precursor for $\mathrm{NADP}^{+}$via NAD kinase found in the cytosol. In addition to G6PD, cytosolic isoforms of ICDH and $\mathrm{MDH}$ may also play important roles in the regeneration of NADPH to prevent oxidative stress (270), at least in some cell types.

\section{Redox-active Cys}

Along with GSH, Cys, and other low-molecular-weight thiols, protein cysteinyl residues represent a large pool of available thiols that can serve as a buffer for oxidant stress. As discussed further below, however, not all protein thiols are affected equally by oxidants, thus allowing for some specificity with regard to the signaling effects of ROS and resulting in reproducible cellular targets for thiol modification by GSH or nitrosating species. Here we examine thiol modifications, their potential functional consequences, and the role of mitochondria in these processes. (See Table 1 for examples that affect mitochondrial function.)

1. Cys oxidation states and functional modification of protein thiols. Hydrogen peroxide promotes cell signaling, in part, via the reversible oxidation of protein thiols. Protein thiols can exist in a number of oxidative states, including sulfenic $(\mathrm{S}-\mathrm{OH})$, sulfinic $\left(\mathrm{S}-\mathrm{O}_{2} \mathrm{H}\right)$, and sulfonic $\left(\mathrm{S}-\mathrm{O}_{3} \mathrm{H}\right)$ acids, as well as disulfide (R-S-S- $\mathrm{R}^{1}$ ) forms (Fig. 4). Thus, key to its usefulness as a signaling mechanism, thiol oxidation is targeted preferentially at critical thiols that are redox active, such as those that exist as thiolate anion ( $\mathrm{RS}^{-}$) under physiological conditions due to the local environment that fosters a decreased $\mathrm{pK}_{\mathrm{a}}$ at this redox-active site (368). Although a simple motif for identifying redox-active thiols is not available, analysis of known modifiable sites suggests a role for nearby noncharged polar residues in the tertiary structure and a possible role for His residues $\mathrm{N}$-terminal to the redox-active Cys (368). With the exceptions of some Prx in which sulfinic acid is reversed enzymatically by Srxs (74), formation of sulfinic acid and the more highly oxidized sulfonic acid are 


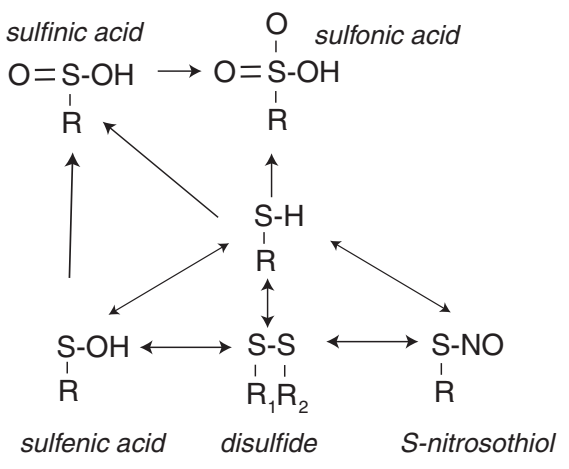

FIG. 4. Thiol oxidation. Protein cysteinyl residues form a large redox-active thiol pool, the modifications of which can regulate protein structure and function $(197,463,464)$. Protein cysteines (Cys) that exist as thiolates $\left(\mathrm{S}^{-}\right)$rather than as S-H may be especially susceptible to modification (not represented in the figure) (368). Oxidative stress can stimulate reversible modification after sulfenic acid or disulfide bond formation. Disulfides can form intramolecularly, between proteins or between a protein and a low-molecular-weight redox active thiol, such as GSH. Trx and Grx may play a role in reducing oxidant-stimulated (reversible) oxidations. Excess oxidant stress is associated with the overoxidation of protein thiols to sulfinic acid or sulfonic acid. These thiol oxidation states are considered irreversible, except for the special case of peroxiredoxins: sulfiredoxin specifically targets and reduces sulfinic acids in some peroxiredoxins (74).

considered overoxidations that are irreversible. In terms of signaling, formation of sulfenic acid or disulfides may result from levels of hydrogen peroxide consistent with receptormediated signaling events. Formation of sulfenic acid, which is unstable, is widely believed to precede disulfide formation (345), which can form between protein thiols or between protein thiols and GSH (S-glutathiolation) or homocysteine (S-homocysteinylation). Essentially, the electrophilic nature of the sulfenic acid increases its susceptibility to nucleophilic attack by thiols or thiolates anions (345). Disulfide bonds are more stable than sulfenic acid and amenable to reduction by Trx (protein disulfides) or Grx (protein disulfides and protein glutathiolations). Recent work suggests that thiols involved in reversible disulfide formation comprise two groups: those with structural and those with functional (thiol-disulfide switch) consequences (464).

The functional consequences of redox-active modifications are only partially understood. Thus, some changes may modify active sites of enzymes, whereas other redox-sensitive changes may occur in sites remote from the active or ligand binding sites that modify enzyme function (allosteric redox modulation) $(197,464)$. The role of thiol oxidation on signal transduction has been studied due to the susceptibility of many protein tyrosine phosphatases to oxidative inactivation by this mechanism. Examples include the phosphatase and tensin homolog (PTEN), which antagonizes growth factor-mediated phosphatidylinositol 3 kinase (PI3K) action to attenuate Akt signaling; PTP1B, which modulates insulin signaling; and mitogen-activated protein kinase (MAPK) phosphatases (MKPs or dual specificity phosphatase [DUSPs]), which target phosphotyrosine as well as phosphoserine/phosphothreonine residues within MAPK (114). All of these pathways are regulated to some extent by ROS produced as a result of growth factor-mediated receptor activation. Oxidative modification of these phosphatases prolongs kinase action, whereas diminished ROS attenuates the oxidative inactivation of these phosphatases to suppress kinase-mediated signaling pathways and lessen sensitivity to receptor-activation.

Mitochondria can be the source of the inactivating ROS as well as the downstream target of ROS-mediated signaling. Thus, in some cells, the growth factor-mediated signal transduction that activates Akt requires mitochondrial output of ROS, as, for example, suppression of mitochondrial ROS attenuates growth factor-mediated phosphorylation of Akt (78, 169). In addition, Akt-activation downstream of growth factor receptor or direct oxidative stimuli appears to be important in promoting mitochondrial biogenesis as inhibition of Aktsignaling can suppress mitochondrial biogenic responses downstream of ROS (330).

Other redox-active proteins are susceptible to sulfenic acid or disulfide formation due to the flux of cellular oxidants (50, $160,234,381,464)$. Some of these modifications have been found under basal conditions due, in part, to oxidants produced during normal respiration in mitochondria, whereas other protein oxidation events are induced after oxidative stress. Within mitochondria, complex I is especially sensitive to redox damage, and thiol oxidation of complex I promotes $\mathrm{O}_{2}^{\bullet-}$ production $(120,416)$, as discussed further below. Other mitochondrial proteins, including the TCA cycle enzymes $\alpha$ $\mathrm{KGDH}$ and aconitase, are especially sensitive to oxidative inactivation (423). Inactivation of $\alpha-\mathrm{KGDH}$ has the more profound effect on NADH generation by the TCA cycle as oxidative inhibition of aconitase can be circumvented by glutamate entry supplying carbons for the TCA cycle following its transamination to $\alpha$-ketoglutarate.

2. Protein S-glutathiolation. Similar to protein disulfides, mixed protein disulfides with glutathione most likely form through a sulfenic acid intermediate whereby formation of R$\mathrm{SO}^{-}$under physiological conditions promotes a reaction with GSH (136). Other mechanisms, such as thiol-disulfide exchange with GSSG, can mediate some S-glutathiolation (-SSG) reactions [reviewed in Ref. (296)]. Protein S-nitrosation can also precede S-glutathiolation, with glutathione replacing the nitroxyl anion (180). Glutathiolation, as a targeted and reversible change, has been suggested to be an adaptive mechanisms that preserves protein function; for example, in the sarcoplasmic-endoplasmic reticulum calcium ATPase (SERCA) pump, loss of glutathiolation of the Cys674 thiol leads to overoxidation of SERCA (421). By contrast, S-SG formation may represent an oxidation reaction that modulates protein function either by reversibly inactivating active sites or by promoting protein activity: Ras family members are activated by -S-SG in vascular smooth muscle cells and other cell types, a process augmented by oxidative stress (2, 95). In mitochondria, glutathiolation of the 51-kDa peptide of complex I is associated with a decrease in complex I activity and an increase in $\mathrm{O}_{2}^{--}$production (76), whereas glutathiolation at other sites in the 75-kDa subunit is associated with a loss in complex I activity but does not increase $\mathrm{O}_{2}^{\bullet-}$ production (181). Interestingly, the S-SG of the 51-kDa peptide was detected following in vivo ischemia/reperfusion injury in the heart, whereas the 75-kDa thiol alterations were not detected following this injury protocol; rather, these changes were only 
detected in studies of isolated mitochondria. Many other proteins, including mitochondrial TCA enzymes, can be inhibited by S-SG modification (388).

The intracellular reduction of mixed protein disulfides is primarily achieved through the action of the Grx enzymes, which, similar to Trxs, can also reduce protein disulfides. Grx requires GSH for the reduction of disulfides and is, therefore, dependent on NADPH and GR to maintain GSH stores (Fig. 3). Of the major forms of Grx, Grx2 targets the mitochondria, although deficiency of either Grx2 or Grx1 decreases mitochondrial complex I activity presumably by redox-dependent mechanisms $(120,209)$. Other studies have similarly found that lack of Grx1 leads to a loss of mitochondrial membrane potential, in part, due to the overoxidation of the VDAC, an outer mitochondrial membrane protein (363) that facilitates flux of $\mathrm{O}_{2}^{\bullet-}$ from mitochondrial sources (168) and may play a role in the formation of the mitochondrial transition pore. In contrast, upregulation of the Grx/GSH/GR system is protective against mitochondrial dysfunction and apoptosis by augmenting signaling cascades and/or preserving mitochondrial function $(119,441)$. Little is known, however, about the proteins protected by upregulation of this reductive pathway as the beneficial actions of the Grx system have not yet been correlated specifically with alterations in protein Sglutathiolation.

3. Protein S-nitrosation. Redox-active thiols can also combine with RNS to form S-nitrosothiols (-S-NO). Similar to -S-SG, -S-NO formation is reversible, targeted to certain redox-active thiols, and capable of altering protein function. For example, S-nitrosation of caspase 3, a proapoptotic enzyme, inhibits its activity to attenuate apoptotic signaling (356). Importantly, many of these modifications can be detected in response to physiological and pathophysiological production of $\mathrm{NO}^{\bullet}$. Thus, in endothelial cells the normal tonic production of $\mathrm{NO}^{\bullet}$ by eNOS is sufficient to cause -S-NO formation (463). Overall, there are three genes encoding NOS isoforms (8): eNOS, expressed primarily in endothelial cells; $i N O S$, which can be induced in a variety of cell types, including macrophages, during the response to cytokines; and neuronal NOS (nNOS), which was first characterized as an encoding neuronal enzyme. nNOS and eNOS isoforms require $\mathrm{Ca}^{2+}$ and calmodulin for their regulation and are constitutively expressed $(1,58$, 366). In contrast, iNOS is transcriptionally induced by cytokines and is regulated by substrate (L-arg) availability (456).

There are several studies that suggest the presence of an NOS isoform in mitochondria (mtNOS); however, the mitochondrial localization of NOS and its nature are highly controversial, with little consensus among different laboratories. Rat liver was first shown to contain an NOS isoform with characteristics similar to those of nNOS and eNOS, as this NOS was found to be $\mathrm{Ca}^{2+}$-dependent and inhibited by L-Arg analogues, such as NG-monomethyl-L-arginine (152). In contrast, another report suggested that liver mitochondrial NOS is most similar to iNOS, although it was found to be constitutively produced (415) rather than induced under stress. Another study countered that although mitochondria contain some eNOS, the majority of mtNOS was a unique form of NOS, as NOS activity was measured in mitochondria from eNOS-deficient mice (231). To add yet another layer to this controversy, other investigators suggested rat liver mitochondria did not contain any NOS isoforms, as neither calmodulin, which is required for NOS activity, nor NOS isoforms were identified in a proteomic analysis of trypsindigested proteins from purified mitochondria (431). In this study, calmodulin or eNOS was readily identified by mass spectrometry when their recombinant forms were added postisolation to purified mitochondria, suggesting an ability to identify trypsin fragments from these proteins by mass spectrometry (431). However, it is impossible to determine whether the lack of $\mathrm{m} / \mathrm{z}$ peaks that can be assigned to calmodulin or NOS was caused by the method of sample processing, or a matter of abundance, or, as reported, evidence for the absence of these proteins. To support their claim that NOS was not in the mitochondrial compartment, these authors also showed negligible NOS activity in isolated liver mitochondria (431). Although recombinant NOS added to these mitochondria had detectable activity (suggesting that there was no inhibitory substance in these preparations), an alternative explanation for the failure to detect NOS activity is that the isolation procedures inactivated the endogenous enzyme. Still, these thorough studies cast serious doubts on the presence of NOS in liver mitochondria. A subsequent study by this group reported that one of the commonly used NOS activity assays that measures radiolabeled $\left[{ }^{14} \mathrm{C}\right]-\mathrm{L}-\mathrm{Arg}$ catabolism to L-citrulline, a byproduct of NO production, can also detect arginase-mediated urea production if insufficient levels of arginase inhibitors are used (432). Briefly, in this NOS activity assay, urea can be coeluted with L-citrulline and thereby provide a false-positive signal for NOS activity. In cardiomyocytes, several reports also propose a role for mitochondrial NOS. In one such study, microsensors were used to monitor the release of NO from single isolated mitochondria, providing some evidence for $\mathrm{NO}$ production in mitochondria (208). Although it was reported that this mtNOS originated from the $n N O S$ gene, other studies of cardiomyocytes suggest that mtNOS is a mixture of iNOS and eNOS (471). A more recent study of cardiac mitochondria similarly suggests that mtNOS is a $\mathrm{Ca}^{2+}$-sensitive enzyme that can be inhibited by the presence of $\mathrm{Mg}^{2+}$ (472). Many of these arguments for or against mtNOS rely on the purity and integrity of the mitochondrial preparations. Although NO is capable of diffusing into mitochondria, the presence of NOS isoforms within mitochondria has significance for mitochondrial functions owing to the ability of NO to nitrosate mitochondrial targets and its ability to combine with superoxide, a byproduct of electron transport, to form peroxynitrite.

Nitric oxide does not directly react with protein thiols; rather, higher oxides of $\mathrm{NO}^{\bullet}$ that are more reactive than $\mathrm{NO}^{\bullet}$ are believed to be the S-nitrosating agents, such as $\mathrm{N}_{2} \mathrm{O}_{3}(199,213$, 312 , 449). Furthermore, nitric oxide can be transferred to protein thiols by transnitrosation. Essentially, low-molecularweight thiols, such as GSH or Cys, or susceptible protein thiols readily become S-nitrosated in vivo and can transfer NO to additional thiol targets (263). Mitochondria play a central role in protein S-nitrosation: many S-nitrosoproteins target to the mitochondria or perimitochondrial regions in cells, and mitochondrial function is necessary for their formation (463). Furthermore, S-nitrosation of mitochondrial targets can modify mitochondrial function $(97,98,474)$.

4. Mitochondrial function and the thiol redox state. Mitochondria are a major source of cellular oxidants, and the basal production of oxidants by mitochondria are essential for 
maintaining the protein thiol redox state of cells, affecting both global S-nitrosation and disulfide formation of proteins (463, 464). Thus, in a staining-based method based on the biotin-switch procedure first developed by Jaffrey and colleagues (191), we detected S-nitrosoproteins in the mitochondrial or perimitochondrial compartments of cells (463). Pretreatment of cells to remove their functional mitochon-

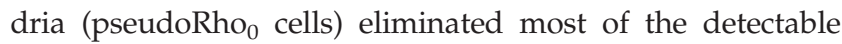
S-nitrosoproteins. Use of mitochondrial inhibitors (rotenone, myzothiazol, or uncouplers) that reduce mitochondrial ROS production diminished S-nitrosoprotein detection, whereas use of antimycin A, which is known to increase ROS production, had little effect on the detection of the Snitrosoproteins, confirming a role for mitochondrial ROS in modification of redox-reactive thiols with $\mathrm{NO}^{\bullet}$. Although the specific modified thiol was not identified within these proteins, proteomic methods allowed for the identification of several S-nitrosated target proteins, many of which have previously been shown to have redox-active thiols.

Other investigators have similarly used a biotin-switchbased method to identify susceptible protein thiols that can become S-nitrosated in whole cells (232) or isolated cardiac mitochondria (305). In these latter studies, following biotin labeling of -S-NO-labeled thiols, proteins were digested with proteolytic enzymes before streptavidin capture and liquid chromatography (LC)/tandem mass spectrometry (MS/MS) analysis of isolated peptides. One advantage of peptide-based purification is that it allows for an identification of the modified site, at least in peptides with a single Cys. The disadvantage is that not every peptide may be amenable to LCbased methods and MS/MS identification. Consistent with our findings, only a subset of available Cys were S-nitrosated in these studies. Although the functional consequences of -S-NO modifications have not been determined in these mapping studies, these data provide useful information regarding possible targets that may mediate the protective and harmful effects of $\mathrm{NO}^{\bullet}$.

In other studies, we found that mitochondrial ROS are also essential for global protein disulfide formation, as inhibitors of mitochondrial ROS significantly reduce disulfide bond formation in cells, as did a mitochondrially targeted catalase (464). From this study, it is not clear whether the disulfides analyzed were due to interprotein, intraprotein, or mixed disulfide (such as with GSH) formation; however, these findings identify a subset of proteins with redox-active thiols that form disulfides under basal conditions. In support of a role for basal respiration in protein disulfide bond formation, studies in yeast, for example, found that anaerobic growth substantially decreased detection of proteins with sulfenic acid or disulfide bonds (234). In yeast, inhibition of the Trxmediated pathways augmented disulfide formation, suggesting a tonic effect of Trx in modulating the extent of protein oxidation under normal metabolic fluxes. Inhibition of GSH synthesis pathways, in contrast, suppressed overall detectable protein disulfide formation, possibly due, in part, to a lack of protein glutathiolation and/or overoxidation of many redoxactive thiols (to sulfinic and sulfonic acid) in an environment lacking GSH. In addition, other researchers have used proteomic methods to study the effects of oxidant stress on protein thiols, finding a subgroup of proteins with increased sensitivity to thiol oxidation $(32,50,51,104)$, including susceptible thiols within the mitochondrial complexes I, II, and
IV (257). Although none of these studies correlated thiol oxidation with mitochondrially produced ROS, they establish a requirement of ROS for their formation and indicate that these redox-dependent events only occur in a subset of the thiol proteome. Our more recent study showed that decreasing cellular ROS through the overexpression of the antioxidant GPx-1 disrupts normal cellular disulfide formation with consequences for mitochondrial function (169). Thus, excess GPx-1, in both the cytosol and the mitochondria, decreases mitochondrial potential and ATP production. Furthermore, this suppression of mitochondrial function contributes to diminished responses in growth factor-mediated Akt activation and cellular proliferation (169). These studies highlight the essential nature of mitochondrial ROS for normal cellular and mitochondrial function.

5. Mitochondrial disulfide-relay system. Similar to the endoplasmic reticulum, enzymes within the mitochondrial intermembrane space catalyze the formation of disulfide bonds in mitochondrially targeted proteins (347). This process has been most widely studied in yeast strains where a disulfide-relay system has been shown to be linked to the import of proteins into the intermembrane space of mitochondria (292). A crucial component of this system is the FADcontaining sulfhydryl oxidase Erv1, which maintains the intermembrane protein Mia40 in an oxidized form $(40,391)$. Mia40 then passes the disulfides to reduced proteins, after their entry into the intermembrane space. Erv1 serves to shuttle electrons from Mia40 to cytochrome c of the ETC in order to maintain its oxidized state $(10,40,105,292)$. GSH plays an important role in this process by preventing the overoxidation of redox-active thiols during disulfide bond formation within the intermembrane space $(40,292)$. The function of this system is less well studied in mammals; however, a mutation in GFER, the human homolog of Erv1, alters the protein content of the mitochondrial intermembrane space, and decreases mitochondrial complex I, II, and III activity (116). This rare human mutation was found to cause a progressive mitochondrial myopathy and congenital cataracts, hearing loss, and developmental delay in affected individuals.

\section{E. Metabolism, NADH/NAD ${ }^{+}$, and NADPH/NADP ${ }^{+}$}

1. Regulation of glycolysis. Within the mitochondria, the enzymes of the TCA cycle supply reducing equivalents $\left(\mathrm{NADH}\right.$ and $\mathrm{FADH}_{2}$ ) for ETC-mediated oxidative phosphorylation and production of ATP. Cellular metabolism of glucose or lipids feeds into the TCA cycle after the formation of acetyl CoA (Fig. 5). The entry of carbons from glucose into the TCA cycle is regulated, in part, by isoforms of lactate dehydrogenase (LDH) that either favor lactate production from pyruvate (lactate dehydrogenase A [LDHA]) or pyruvate formation from lactate (lactate dehydrogenase B [LDHB]) (276). The LDHA production of lactate results in oxidation of $\mathrm{NADH}$ (pyruvate $+\mathrm{NADH} \rightarrow$ lactate $+\mathrm{NAD}^{+}$) whereas the $\mathrm{LDHB}$ production of pyruvate causes the reduction of $\mathrm{NAD}^{+}$. Certain glycolytic cells may also secrete lactate that is taken up by other neighboring cells. For example, in brain, lactate secreted by astrocytes is taken up by the monocarboxylate transporter into neurons where it is used as a fuel source (117). Other cells can similarly utilize lactate as an energy source following its conversion to pyruvate. Pyruvate 


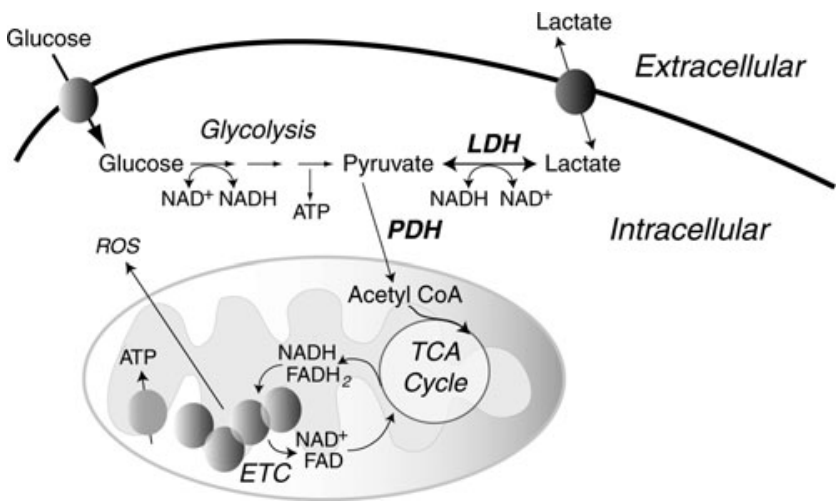

FIG. 5. Glucose metabolism and NADH/NAD ${ }^{+}$. The enzymes, pyruvate dehydrogenase $(\mathrm{PDH})$ and lactate dehydrogenase $(\mathrm{LDH})$, dictate whether glycolysis proceeds to glucose oxidation or the formation of lactate. $\mathrm{PDH}$ can be inhibited by fatty acid oxidation (341), c-jun N-terminal kinase (JNK) signaling (480), and hypoxia-mediated upregulation of PDH kinase, which, similar to JNK, phosphorylates and inactivates $\mathrm{PDH}$ (386). $\mathrm{LDH}$ activity is dependent on the expression of the lactate dehydrogenase A (LDHA) or lactate dehydrogenase B (LDHB) subtypes, which promote either lactate formation or pyruvate formation, respectively. The absence of glucose or other conditions that elevate $\mathrm{NAD}^{+}$ promotes sirtuin (SIRT) deacetylase activity to stimulate respiration, activate glycolytic genes, and increase fatty acid oxidation (219) (not shown in figure).

dehydrogenase $(\mathrm{PDH})$ is another enzyme that regulates the utilization of pyruvate; it is itself regulated by $\mathrm{PDH}$ kinase (PDK), becoming inactivated by phosphorylation. It is well established that high rates of fatty acid oxidation inhibit glucose oxidation by inhibition of PDK, in part, due to high $\mathrm{NADH} / \mathrm{NAD}^{+}$; the reverse is also true: low rates of fatty acid oxidation promote glucose oxidation $(341,400)$. These mechanisms suggest mitochondrial adaptation to available substrates (Randle cycle); however, alterations in the normal balance between glucose and fatty acid oxidation has been suggested to contribute to excessive cellular proliferation, as is seen in some cancers and in pulmonary hypertension (408, 429). Redox activation of c-jun N-terminal kinase (JNK) also fosters a decrease in PDH activity by increasing its phosphorylation (480) to limit TCA-mediated NADH production and subsequent ETC activity. Loss of pyruvate-mediated, high-yield energy production due to deficiencies in the TCA cycle and oxidative phosphorylation determinants is found in aging, neurodegenerative diseases, and also in cancer and during hypoxia. In type 2 diabetics, disruption of normal ETC activity in skeletal muscle may also contribute to a reduction of ATP generation (351). Although some cells are more glycolytic in nature (ending glycolysis with the production of lactate, rather than the complete oxidation of pyruvate in the TCA cycle), hypoxia mediates a glycolytic shift to anaerobic glycolysis caused by the coordinate upregulation of proteins such as LDHA and PDH kinase by the hypoxia-inducible transcription factors, especially hypoxia-inducible factor-1 (HIF-1), resulting in lactate production and an $\mathrm{NAD}^{+}$increase $(385,386)$. The corresponding increase in glucose uptake by the upregulation of the glucose transporter-1 (Glut1) helps to maintain the cellular $\mathrm{NADH} / \mathrm{NAD}^{+}$ratio and provides an energy source for this anaerobic glycolysis. (During anaerobic glycolysis, cytosolic NADH produced by glyceraldehyde-3phosphate dehydrogenase is recycled into $\mathrm{NAD}^{+}$by LDHA production of lactate from pyruvate, whereas during the complete oxidation of glucose, pyruvate entry into the TCA cycle results in NADH production that is oxidized by respiratory complex I.) Importantly, recent work with selective genetic sensors for cytosolic and mitochondrial NADH suggests that, in contrast to the cytosolic compartment, cells have a strong tendency to maintain mitochondrial NADH homeostasis in response to a variety of oxidant stresses (477). The coordinate metabolic changes caused by HIF-1 that alter mitochondrial function are attributable, in part, to the HIF-1mediated transcriptional upregulation of microRNA 210 (miR210), which specifically inhibits Fe-S complex formation to alter mitochondrial bioenergetics and ROS production (Pasteur effect) (69). These pathways are discussed further below.

2. SIRT, $\mathrm{NAD}^{+}$, and metabolic regulation. Although there are a number of $\mathrm{NAD}^{+}$-requiring enzymes in mammalian cells (219), the SIRT, a family of NAD ${ }^{+}$-dependent protein deacetylases that target lysine residues on histones, nuclear factors, and other proteins, have the greatest effect on coordinating metabolism and mitochondrial function. In mammals, this family has several members of which SIRT1, SIRT6, and SIRT7 are nuclear proteins; SIRT1 is found mainly in the cytoplasm; and the SIRT3, SIRT4, and SIRT5 are found in the mitochondria $(165,295,389)$. SIRT1 is known to be activated in response to metabolic changes, such as low glucose or exercise, that increase cellular $\operatorname{NAD}^{+}(62,354)$. SIRT1 can also be activated by AMP kinase (AMPK), which is a metabolic sensor of low ATP. SIRT1 has been shown to target the deacetylation of PGC- $1 \alpha$, which may influence mitochondrial biogenesis and increase mitochondrial respiration (33), in part, by enhancing fatty acid oxidation (150) (Fig. 6). Similarly, other SIRT family members have been found to affect metabolism. SIRT6 activation coordinately regulates the expression of glycolytic genes (479), and mice deficient in SIRT6 have severe metabolic defects, signs of accelerated aging, and die prematurely. The mitochondrial SIRT3 has been found to modulate mitochondrial function, possibly by targeting the deacetylation of mitochondrial complex I or other mitochondrial proteins, including ICDH-2, a potential source for NADPH $(6,389,394)$. Thus, SIRT3 activity may decrease oxidative damage by increasing mitochondrial NADPH to allow for GR and TrxR reduction of GSH and oxidized Trx, respectively (394). In mice, SIRT3 is normally upregulated during fasting in liver and brown fat where it plays a role in fatty-acid oxidation by maintaining the deacetylation of longchain acyl coenzyme A dehydrogenase in mitochondria (175). In its absence, SIRT3-deficient mice show reduced utilization of lipid stores during fasting: they have decreased fatty acid oxidation, accumulate triglycerides, and have diminished production of hepatic ATP.

Owing to their growing importance as metabolic regulators and the findings that SIRT homologs in Caenorhabditis elegans and Drosophila melanogaster may extend lifespan $(201,419)$, there is an interest in developing pharmaceutical mediators of SIRT function. There is, however, some recent controversy regarding the role of SIRT in extending lifespan, in that recent reports fail to show robust effects of SIRT on lifespan in these same model systems (56). Nonetheless, these NAD ${ }^{+}$- 


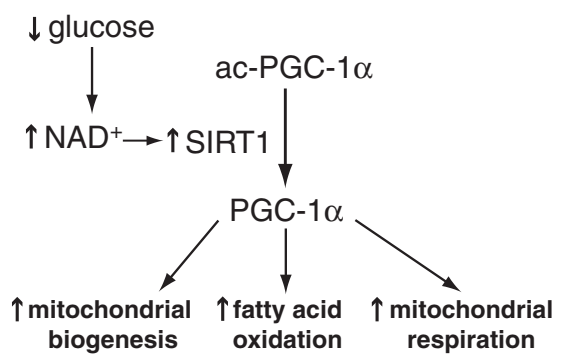

FIG. 6. Regulation of metabolism by SIRT1. SIRT can affect mitochondrial function in several ways. SIRT1 has been shown to modulate mitochondrial biogenesis and fatty acid oxidation $(62,354)$. A decrease in cellular glucose leads to an increase in intracellular $\mathrm{NAD}^{+}$to promote SIRT1 deacetylase activity. Peroxisome proliferator-activated receptor- $\gamma$ coactivator- 1 alpha (PGC- $1 \alpha$ ) is one of the targets of SIRT1 (33). Deacetylation of this transcriptional coactivator promotes the biogenic program (in part, via nuclear respiratory factors, not represented in this diagram) to increase cellular mitochondrial content (150). In addition, in highly metabolic cells, such as skeletal muscle, PGC- $1 \alpha$ activation increases the expression of genes involved in fatty acid oxidation to increase mitochondrial respiration (150). Activation of other SIRTs, such as SIRT3, may also contribute to these pathways (not shown in the diagram). SIRT3 targets to mitochondria, and its deacetylation of mitochondrial proteins may augment complex I activity $(6,389)$ and increase fatty acid oxidation by maintaining the activation of the longchain acyl coenzyme A dehydrogenase, at least in liver (175).

responsive genes serve to coordinately regulate metabolism and mitochondrial function. AMPK mechanisms apparently upregulate SIRT function (62), as does resveratrol $(33,44)$. A more recent study used pharmacological methods to induce the oxidation of NADH by the cytosolic NADH:quinone oxidoreductase 1 (NQ1) (183). NQI is an obligate two-electron reductase that participates in cellular antioxidant protection by reducing reactive quinones to hydroquinones avoiding the formation of free radical semiquinones. In this study, $\beta$-lapachone supplementation was found to increase intracellular $\mathrm{NAD}^{+}$and, in vivo, this treatment decreased adiposity, dyslipidemia, glucose intolerance, and fatty liver in diet-induced obesity as well as the ob/ob obese mouse. Furthermore, these metabolic improvements correlated with increased gene expression of some SIRT family members and with upregulation of PGC- $1 \alpha$ and nuclear respiratory factor- 1 (NRF1), two factors involved in mitochondrial biogenesis (see Table 1 for examples of acetylation that modulate mitochondrial function). Consistent with the upregulation of these biogenic factors, $\beta$-lapachone supplementation also increased skeletal muscle mitochondrial content as well as oxygen consumption. Although the increase in $\mathrm{NAD}^{+}$indirectly implicates SIRT-mediated activation, protein acetylation and SIRT activity were not assessed in this study. Nonetheless, these findings suggest a dramatic metabolic shift caused by modification of intracellular $\mathrm{NADH} / \mathrm{NAD}^{+}$ratio. It should be mentioned, however, that $\beta$-lapachone and other similar quinones are used chemotherapeutically for their ability to cause tumor cell death, which has been attributed to oxidative stress caused, in part, to the decrease in cellular NADH/ $\mathrm{NAD}(\mathrm{P}) \mathrm{H}$ pools and/or the production of toxic forms of hydroquinones that generate ROS (332).

\section{Functional Consequences of Redox Modifiers}

\section{A. $N O^{\circ}$}

Physiological, pathophysiological, and pharmacological levels of $\mathrm{NO}^{\bullet}$ can mediate protein $-\mathrm{S}-\mathrm{NO}$ formation. In addition, $\mathrm{NO}^{\bullet}$ may affect cellular functions via activation of soluble guanylyl cyclase (sGC) and the latter's activation of cGMP-mediated protein kinase G pathways that involve protein phosphorylation rather than protein S-nitrosation. Nitric oxide may also promote protein modification through nitration of tyrosines to form the stable 3-nitrotyrosine. Accumulation of 3-nitrotyrosine in proteins is indicative of ROS and RNS stress (188). In this section we next consider evidence for protein -S-NO modifications that specifically alter mitochondrial function (Fig. 7).

1. Apoptosis and $\mathrm{NO}^{\bullet}$. At low concentrations, $\mathrm{NO}^{\bullet}$ is considered protective against apoptosis, whereas at higher concentrations, such as those that can be generated during inflammatory states, it can mediate apoptotic responses due, in part, to the formation of peroxynitrite $(281,439)$. The beneficial or harmful effects of $\mathrm{NO}^{\bullet}$ may also depend on the cell type, duration and intracellular location of its production, and redox status of the cell. We found that bovine aortic endothelial cells grown in the presence of an $\mathrm{NO}^{\bullet}$ donor at $1 \mathrm{mM}$ causes a decrease in mitochondrial membrane potential, with the release of cytochrome $c$ and activation of caspase cascades (439). Furthermore, hypoxia augments these effects, whereas uric acid, a peroxynitrite scavenger, attenuates these responses. In

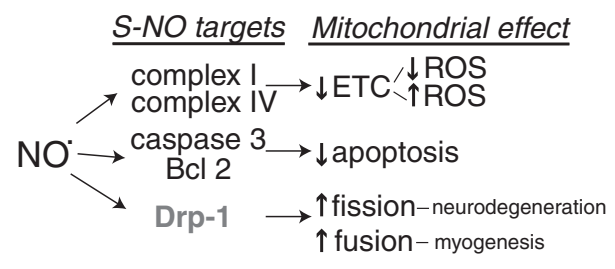

FIG. 7. S-NO targets and mitochondrial function. Nitric oxide $\left(\mathrm{NO}^{\bullet}\right)$ can mediate protein S-nitrosation (-S-NO) or activate soluble guanylyl cyclase to foster cGMP-mediated kinase function. Protein $-\mathrm{S}-\mathrm{NO}$ has been shown to occur in response to physiological and pathological production of $\mathrm{NO}^{\bullet}(463)$, and $\mathrm{NO}^{\bullet}$ has been shown to regulate mitochondrial function through several mechanisms. Complex I Snitrosation has been associated with diminished activity; however, conflicting results exist as to whether it inhibits or promotes mitochondrial ROS generation $(57,98,108)$. Similarly, S-nitrosation of complex IV has been suggested to inhibit mitochondrial respiration $(97,474)$. In general, low concentrations of $\mathrm{NO}^{\bullet}$ are associated with antiapoptotic mechanisms, perhaps, in part, via the inhibition of caspase 3 activation or the decreased degradation of the antiapoptotic Bcl-2 following their respective S-nitrosation $(21,424)$. Nitric oxide may enhance cell death and mitochondrial fragmentation in neurodegenerative diseases by its effects on dynamin-related protein-1 (Drp1), which promotes mitochondrial fission (86). There is some controversy, however, as to whether these are cGMP-kinase-mediated or -S-NO-specific effects (45). In addition, in myogenesis, $\mathrm{NO}^{\bullet}$ has the opposite effect on mitochondrial fission, promoting the fusion of mitochondria into an elongated network by inhibiting Drp1mediated fission (113). 
an in vivo model of neuronal apoptosis due to target deprivation (a model which replicates changes thought to occur in development, or following brain injury or degeneration in which loss of a neuron's target fosters neuronal death due, in part, to loss of retrograde stimulation from the innervated target), intracellular generation of $\mathrm{ROS}$ and $\mathrm{NO}^{\bullet}$ promotes protein modifications like S-NO and 3-nitrotyrosine formation in mouse brain (277). In this model, apoptosis was absent in mice deficient in $\mathrm{nNOS}$ or those treated with NOS inhibitors (277), implicating $\mathrm{NO}^{\bullet}$ in the apoptotic mechanisms. Although these studies did not identify -S-NO targets specifically, there are a number of studies that correlate the pro- and antiapoptotic mechanisms of $\mathrm{NO}^{\bullet}$ with S-nitrosation of protein targets that directly or indirectly affect mitochondrial function. Effector caspases mediate the pathways that lead to cell death, including chromatin condensation and DNA fragmentation, and are activated in both the extrinsic (death-receptor mediated) and intrinsic (mitochondrial) pathways of apoptosis (54). Caspase 3-S-nitrosation is antiapoptotic as it inhibits the activation of this effector caspase (424). In the intrinsic pathway of apoptosis, Bcl-2 prevents the mitochondrial release of pro-apoptotic factors, such as cytochrome c, by forming heterodimers with pro-apoptotic proteins, such as Bax. S-nitrosation of $\mathrm{Bcl}-2$ prevents the proteasomal degradation of this antiapoptotic protein after apoptotic stimuli to enhance its antiapoptotic function (21). The mitochondrial inner membrane adenine nucleotide transporter (ANT) may also be a target for S-nitrosation (305). Although the functional consequences of ANT S-nitrosation in cells has not been demonstrated, it is known that modification of redox-sensitive thiols in this mitochondrial protein regulates the formation of the mitochondrial permeability transition (MPT) pore and cell death (101). Redox regulation of ANT and MPT pore formation is discussed further below.

2. $\mathrm{NO}^{\bullet}$ and mitochondrial fission and fusion. Nitric oxide has also been found to alter intracellular mitochondrial networks. Briefly, mitochondria exist as a dynamic network, the nature of which may be regulated by several factors, including interaction with the cytoskeleton and fusion and fission $(93,138)$. It is believed that alterations in mitochondrial fusion and fission may serve functional purposes to respond to oxygen and metabolic fluctuations $(24,446)$. The significance of these rearrangements in mitochondrial architecture are not entirely clear; studies suggest that ROS may promote mitochondrial fission (457), whereas other studies suggest that fission may promote mitochondrial generation of ROS (387). Possibly, changes in mitochondrial network structure provide an example of ROS-mediated ROS generation where ROS plays a role in mitochondrial fission to augment ROS generation from restructured mitochondria. The relationship between mitochondrial network formation and bioenergetics is not well understood. In cell culture systems, inhibition of oxidative phosphorylation is known to promote mitochondrial fission; yet, not all genetic defects in oxidative phosphorylation alter mitochondrial network formation, suggesting a more complex relationship between bioenergetics and mitochondrial dynamics (371). Furthermore, the effects of mitochondrial dynamics on bioenergetics are unresolved. In the process of myogenic differentiation, $\mathrm{NO}^{\bullet}$ induced mitochondrial elongation (fusion) is a necessary step in myogenesis that appears to require repression of the pro- fission GTPase dynamin-related protein-1 (Drp1) (113). Blocking these necessary $\mathrm{NO}^{\bullet}$-mediated pathways in differentiating myoblasts significantly alters mitochondrial function and reduces oxidative phosphorylation. Drp1 was also proposed as a direct target for S-nitrosation to promote the toxic effects of $\beta$-amyloid in Alzheimer disease due to an NOmediated enhancement of Drp1 activity that promotes mitochondrial fission and subsequent fragmentation (86). A later study, however, challenged the role of Drp1 S-nitrosation in Alzheimer disease, suggesting, instead, that $\mathrm{NO}^{\bullet}$ promotes activation of Drp1 by phosphorylation rather than $-\mathrm{S}-\mathrm{NO}$ modification as -S-NO forms of Drp1 are detectable in normal brains as well as those from Parkinson and Alzheimer diseases (45). Thus, this study supports a role for sGC/cGMP signaling pathways rather than S-nitrosation in modulating mitochondrial fission. Nonetheless, in neurons, $\mathrm{NO}^{\bullet}$ production from nNOS is increased in response to $\beta$-amyloid oligomers, and upregulation of $\mathrm{NO}^{\bullet}$ production and S-NO formation are common features of other neurodegenerative diseases, including Parkinson disease, Huntington disease, ALS, and stroke (218). In addition, excess $\mathrm{NO}^{\bullet}$ production has been shown to promote mitochondrial fission in neurons (28), an effect opposite of its role as a factor that promotes elongation of mitochondria during myogenesis (113). Further analysis to elucidate the pathogenic role of $\mathrm{NO}^{\bullet}$ in neurodegeneration may lead to useful development of drugs to modulate these processes, perhaps by targeting $\mathrm{nNOS}$ or its neuronal associated pathways. Additional research indicates that GSH is an important mediator of $\mathrm{NO}^{\bullet}$ cytotoxicity in neurons as depletion of $\mathrm{GSH}$ enhances $\mathrm{NO}^{\circ}$-mediated apoptosis by enhancing mitochondrial apoptosis-inducing factor (AIF) release (15). This study further finds that enhanced SOD1 overexpression is protective against these changes, suggesting the usefulness of (targeted) antioxidant agents in protecting against the damaging effects of excess neuronal $\mathrm{NO}^{\bullet}$.

3. $\mathrm{NO}^{\bullet}$ and mitochondrial respiration. As mentioned above, $\mathrm{NO}^{\bullet}$ can target and modify subunits of the ETC complexes to reduce their activities. Mapping of $-\mathrm{S}-\mathrm{NO}$ sites in mitochondria identified seven potential sites within six subunits of complex I and as well as other sites of S-nitrosation in complexes I and II (305). Earlier studies had previously provided evidence that exogenous $\mathrm{NO}^{\bullet}$ donors could inhibit complex I in whole cells by a mechanism that can be attenuated by low-molecular-weight thiol antioxidants, such as dithiothreitol or GSH, or by light of the appropriate action spectrum for $-\mathrm{S}-\mathrm{NO}$ species, suggesting a role for $-\mathrm{S}-\mathrm{NO}$ formation in this inhibitory effect (98). Using similar methods, other studies have found a role for $\mathrm{NO}^{\circ}$ in the inhibition of complex IV (cytochrome c oxidase) $(97,474)$ or in the ATPase complex (405). Studies of the effects of $\mathrm{NO}^{\bullet}$ in endothelial cells identified two Cys of porcine complex IV as targets of inactivating -S-NO modification (474). Other studies of isolated mitochondria suggest that S-nitrosothiols such as GSNO are better donors of $\mathrm{NO}^{\bullet}$ for transfer to redox-active thiols than other forms of $\mathrm{NO}^{\bullet}$ donors (108). Many of the above studies were performed using exogenous $\mathrm{NO}^{\bullet}$ donors; however, other studies have found that some of these -S-NO modifications can be detected in isolated whole heart models (Langendorff preparations) of cardiac function. Using Langendorff perfused hearts, endogenously produced $\mathrm{NO}^{\bullet}$ was 
found to cause S-nitrosation of complex I during ischemic preconditioning (57), a process in which hearts are subjected to intervals of brief ischemia and reperfusion to stimulate innate protective mechanisms against injury resulting from more prolonged ischemia/reperfusion. It is generally believed that mitochondrial dysfunction and release of oxidants contribute to the damage accompanying reperfusion injury. Thus, it has been hypothesized that the -S-NO-mediated inhibition of mitochondrial respiration may be cardioprotective (57). Based on this premise, mitochondrially targeted S-nitrosothiols have been used as protective agents against ischemia-reperfusion injury to slow the burst of respiration (and oxidants) produced during reperfusion. In separate studies, pre-ischemic (306) or postischemic (336) use of these reagents lessened ischemia-reperfusion injury, suggesting these methods may be promising in terms of limiting cardiac reperfusion injury after myocardial infarction. One possible advantage of these treatments is the labile nature of -S-NO, thus suggesting that any effects are temporary. These S-nitrosating compounds, however, target other mitochondrial proteins as well (91), and may have other cellular effects neither completely understood nor well characterized. Therefore, there is a need to explore in more detail the consequences of modifying ETC and other mitochondrial targets in vivo. Earlier studies, for example, linked S-nitrosation of complex I with an increase in production of $\mathrm{O}_{2}^{\bullet-}$, at least in isolated respiring mitochondria (108), and, as discussed above, excess $\mathrm{NO}^{\bullet}$ can promote pro-apoptotic events, all highlighting the complexity of these interrelated processes and their biochemistry.

\section{B. Reactive oxygen species}

Under some conditions, upregulation of cellular ROS production has been found to increase further ROS production (ROS-mediated ROS generation) by augmenting mitochondrial production of ROS. There are many contributors to this effect, some of which are further addressed in this section. Additionally, we examine other modulators of mitochondrial ROS, including growth factor receptor signaling and uncoupling proteins (UCPs), which serve to lessen mitochondrial ROS through their induction of a proton leak against the mitochondrial proton gradient.

1. NADPH activation of mitochondrial ROS. Several studies have implicated ROS and mitochondrial damage in the pathogenic effects of AII $(111,112,300)$. AII is known to stimulate NOX activation, but a role for mitochondrially derived oxidants, downstream of AII-mediated receptor activation, is also emerging $(121,215)$. In fact, AII-activated NOXs were found to cause mitochondrial dysfunction and upregulate mitochondrial release of oxidants, in part, by stimulating ETC-mediated production of ROS (121). Interestingly, peroxynitrite formation was shown to be involved in these pathways as uric acid, a peroxynitrite scavenger, blocked mitochondrial ROS generation and mitochondrial dysfunction, attenuating AII-mediated reduction of $\Delta \Psi$, and decreased the respiratory control ratio, a measure of coupling between respiration and phosphorylation. Mitochondrial potassium channels were also implicated in enhanced mitochondrial ROS generation and increased uncoupling. Furthermore, in these studies, there was a concurrent decrease in GSH levels in the mitochondria and cytosol, indicating overall redox stress caused by the increase in cellular ROS. In support of a role for GSH-mediated oxidation in these events, other studies examining the effects of GSH/GSSG redox status on mitochondrial function found that a more oxidative ratio correlated with mitochondrial uncoupling and a decrease in oxidative phosphorylation-mediated ATP generation $(13,174)$.

Of the NOX isoforms, NOX4 has been uniquely found to target to mitochondria in some cells, although there is controversy regarding the intracellular compartment in which NOX4 is localized. In human endothelial cells, NOX4 was found in the endoplasmic reticulum, but this study did not specifically examine mitochondrial localization by proteinbased methods. Rather, the investigators used in silico analysis (PSORTII) to suggest that NOX4 lacks a mitochondrial localization sequence and is more likely to be found in endoplasmic reticulum fractions than other subcellular compartments (77). In mouse heart, however, endogenous NOX4 has been found in both mitochondria and microsomal fractions (4). This report of a cardiac mitochondrial NOX4 also provided experimental evidence that the N-terminal region of NOX4 is capable of targeting proteins to the mitochondria. To date, it is unclear whether NOX4 has the same distribution in all cell types. In mouse hearts, chronic treatments with pressor agents (AII, phenylephrine) and transverse aortic constriction (TAC) were found to increase NOX4 expression. In addition, cardiac expression of NOX4 was found to increase during normal aging (4). Notably, the increase in cardiac mitochondrial ROS following these hypertrophic stimuli was found to be due to both increases in NOX4 expression as well as enhanced mitochondrial-dependent ROS production, suggesting a relationship between the enhanced expression of NOX4 and mitochondrial ROS production and dysfunction. In support of a role for NOX4 in altering mitochondrial ROS production and function, forced upregulation of mitochondrial NOX4 in the heart, by using a targeted transgene, increased thiol oxidation of many mitochondrial proteins including the ETC complex I, aconitase, and other TCA enzymes (4). Some of these targets were also found to be oxidized after TACinduced hypertrophy. Interestingly, cardiac overexpression of NOX4 also caused a decrease in the mitochondrial biogenic signal in the heart, resulting in decreased levels of mitochondrial DNA (mtDNA) in cardiomyocytes from these overexpressing mice. Several studies suggest that ROS may be involved in retrograde signaling from mitochondria to the nucleus to stimulate the biogenic response, possibly as a means to replace oxidized mitochondrial proteins or DNA. Retrograde signaling may also help to coordinate growth factor responses to augment mitochondrial production of energy (350). Possibly, in the case of chronically overexpressed NOX4, excess ROS may reach a threshold at which it promotes apoptotic rather than biogenic responses. As discussed further below, the extent of redox imbalance and oxidant damage may also dictate the choice between the normal replacement of mitochondria, which may additionally include the targeted removal of damaged mitochondria by mitophagy, and cell death via apoptosis.

2. Other ROS-mediated ROS generators. The above studies suggest the utility of suppressing AII signaling and/ or NOX upregulation as means of protecting against ROSinduced mitochondrial dysfunction. Other studies indicate 
that mitochondrial complex I is especially sensitive to redox damage, and that thiol oxidation of complex I promotes $\mathrm{O}_{2}^{\bullet-}$ production $(120,416)$, suggesting the utility of using targeted antioxidants to block mitochondrial dysfunction. Upregulation of UCP expression or activation can also lessen mitochondrial oxidant production (22). An oxidant burst in one mitochondrion may also serve to spread oxidant production throughout the mitochondrial network in a manner that involves transient MPT pore formation, waves of reduction in mitochondrial potential, and propagation of oxidant bursts, at least in cardiomyocytes $(48,481)$.

Excess oxidants may additionally augment mitochondrial ROS by upregulating the expression of the lifespan regulator p $66^{\text {shc }}$ to the mitochondrial intermembrane space (297). Within the mitochondria, $\mathrm{p} 66^{\mathrm{Shc}}$ increases oxidant production by oxidizing cytochrome c to produce hydrogen peroxide and activate the mitochondrial transition pore (153). Furthermore, knockout of $\mathrm{p} 66^{\mathrm{Shc}}$ increases lifespan, reduces hydrogen peroxide generation, and enhances survival to oxidant stress (297). Crucial to the apoptotic function of this protein are two redox centers in the $\mathrm{N}$-terminus: one center plays a role in the copper-dependent catalytic site involved in ROS formation, and the other center is important for tetramer formation, which appears to be essential for the apoptotic function of this protein (151). Intracellular antioxidants such as Trx or GSH are thought to maintain the mitochondrial form of $\mathrm{p} 66^{\mathrm{Shc}}$ in an inactive state.

Mitochondrial dysfunction and excess production of oxidants have been considered contributors to the development of the metabolic syndrome and diabetes. Remarkably, p66 $6^{\mathrm{Sh}}$ deficient mice have been found to be resistant to many complications of experimental diabetes, including diabetic nephropathy, diabetic cardiomyopathy, cardiac stem cell death, hyperglycemia-induced endothelial dysfunction and enhanced lipid peroxidation, and impaired wound healing $(61,128,291,357)$. Furthermore, in high glucose or AII-mediated models of renal tubular injury, $\mathrm{p} 66^{\text {Shc }}$ was found to cause injury by oxidative mechanisms involving mitochondrial dysfunction (increased ROS, decreased mitochondrial potential, decreased mtDNA) and stimulation of apoptotic pathways involving increased cytochrome c release, caspase 9 activation, and mtDNA damage (406).

3. Extracellular redox state and mitochondrial function. In the intracellular environment GSH/GSSG is the major redox couple but, extracellularly, in human plasma, Cys and cystine (CySS) are the predominant low-molecularweight thiol redox determinants (155). In the oxidized extracellular environment, there is more CySS than Cys. The Cys/ CySS ratio tends to become even more oxidized with aging, smoking, diabetes, and obesity $(198,302)$. Recent findings also suggest that modifying the Cys/CySS ratio in cell culture systems can enhance inflammatory responses and modulate cell growth (192). Apparently, the Cys/CySS ratio can affect the intracellular redox balance, altering the oxidation of mitochondrial Trx2, leading to the increased production of mitochondrial $\operatorname{ROS}(157,184)$. Redox-active thiols accessible at the plasma membrane were found to mediate these effects through mechanisms that may involve receptor proteins and subsequent signaling-dependent oxidation of actin and other intracellular protein targets (157). A role for redox mechanisms in receptor-mediated activation has previously been shown $(89,207)$; however, more research is needed to understand these mechanisms and their role in modulating cellular function.

4. Mitochondrial ROS and receptor-mediated signaling. The mitochondrial generation of ROS downstream of receptor signaling is an essential step in signal transduction (Fig. 8). As discussed above and in Figure 8, ROS generated by mitochondria contribute to phosphatase inactivation and promote cell signaling. Furthermore, mitochondrially produced ROS can activate insulin receptor autophosphorylation after insulin stimulation to promote receptor-mediated actions (402). Suppression of mitochondrial ROS, either by inhibiting mitochondrial function with uncouplers or by decreasing ROS release with scavengers or antioxidants, significantly reduces receptor-mediated signaling. In support of the notion that ROS modulates receptor signaling, siRNA knockdown of GPx-1 in cultured cells significantly potentiates epidermal growth factor (EGF)-stimulated Akt phosphorylation, whereas overexpression of GPx-1 or catalase suppresses these pathways (169). Furthermore, in mouse in vivo models, lack of GPx-1 was found to augment insulin-mediated Akt

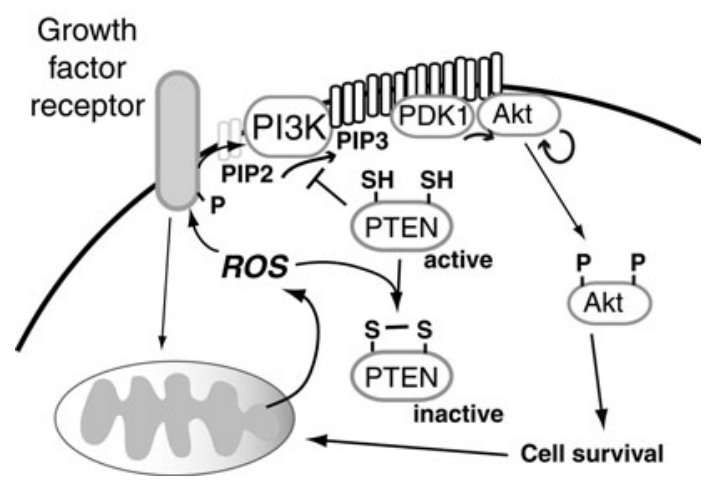

FIG. 8. The role of mitochondria in growth factormediated signaling. Growth factor receptor signaling promotes mitochondrial ROS, without which signaling responses are diminished (169). Mitochondrial ROS serve to activate receptor phosphorylation (402) and promote intracellular signaling, in part, by the oxidative inactivation of phosphatases (114). Illustrated in this figure is the role of ROS in Akt signaling, a process in which mitochondrial ROS are essential (269). Phosphatidylinositol 3 kinase (PI3K) phosphorylates membrane phosphatidylinositol-4,5-phosphate (PIP2) to PIP3 (3,4,5 phosphate). Accumulation of PIP3 in the membrane recruits PDH kinase 1 (PDK1) and Akt to the membrane, where PDK1 phosphorylates Akt (269). Akt may also be phosphorylated by other kinases, or self-phosphorylate to a certain degree. Activated Akt targets other cellular proteins to promote cell survival and may also enhance mitochondrial biogenesis. The actions of PI3K are antagonized by the lipid phosphatase phosphatase and tensin homolog (PTEN), which dephosphorylates PIP3. PTEN is inactivated by redox modification (330): in the presence of hydrogen peroxide, PTEN forms an intramolecular disulfide bond. Oxidative inactivation of PTEN promotes growth factor signaling. Similarly, oxidative inactivation of other phosphatases, such as PTP1B or MAP kinase phosphatases, is necessary for essential signal transduction downstream of insulin-mediated receptor activation or other signaling events, respectively. 
activation in skeletal muscle (264), whereas overexpression of GPx-1 attenuated these responses in transgenic mice (284). Mitochondrial dysfunction is believed to play a role in type 2 diabetes, which is characterized by insulin resistance and dysregulation of glucose and lipid metabolism. Thus, a lack of response to insulin may ensue by a loss of beneficial ROS production in response to receptor-mediated events. One thought is that mitochondrial insufficiency may result in a blunted production of essential ROS necessary for signal transduction in diabetics $(210,325,434)$. Even though such a concept is supported by some experimental data, this view is controversial. Many studies implicate, instead, mitochondrial dysregulation of metabolism in diabetes that may coincide with mitochondrial generation of excess ROS, which exceeds a threshold compatible with normal responses to insulin [insulin signaling, metabolism and mitochondria are reviewed in Ref. (83)].

5. Mitochondrial respiration, inner mitochondrial membrane potential, and ROS. The outer membrane of the mitochondria is freely permeable to molecules $<5000 \mathrm{kDa}$, in contrast to the inner mitochondrial membrane, which is highly impermeable. As discussed in section II.B, during respiration, protons are pumped into the intermembrane space to create a gradient of $\mathrm{H}^{+}$, which is the major contributor to the mitochondrial inner membrane potential $(\Delta \Psi)$; concurrently, $\mathrm{O}_{2}^{--}$is produced due to electron leak in the ETC. The electrochemical gradient caused by proton accumulation in the intermembrane space is utilized to drive the synthesis of ATP at the ATP synthase complex. Some proton leak back through the inner mitochondrial membrane (mild uncoupling) serves to reduce the production of $\mathrm{O}_{2}^{\bullet-}$ and increase the respiration rate. As discussed further in the next section, upregulation and activation of UCPs serve to lessen $\mathrm{O}_{2}^{\bullet-}$ by increasing the leak of protons back into the matrix. Inner mitochondrial potential is high when ROS production is high and high ROS can subsequently lead to decreased $\Delta \Psi$ to lessen detrimental ROS production, by increasing proton leak, at the cost of reducing ATP production (52). Increased proton leak results in lower $\Delta \Psi$ (and lower ATP) for any $\mathrm{O}_{2}$ consumption rate. As mentioned in section II.C.5., $\Delta \Psi$ also drives the activity of NNT to maintain matrix NADPH, which is necessary for mitochondrial antioxidant enzyme systems (226). NADH from the TCA cycle is also essential for NNT production of NADPH. Loss of antioxidant pathways from decreased $\Delta \Psi$ may explain why in oxidatively stressed cardiomyocytes uncoupling of oxidative phosphorylation paradoxically increases ROS (14). These findings suggest that the redox environment modulates the effect of mitochondrial uncoupling.

6. UCPs and mitochondrial function. UCP1 plays a role in adaptive thermogenesis in mammalian brown adipose tissue to produce heat (314), whereas the actions of UCP2 and 3 are upregulated in response to oxidant stress caused by nutrient deprivation, glucose sensing, fatty acid metabolism, or other metabolic changes in order to lessen mitochondrial ROS production (49). These anion carriers lessen mitochondrial ROS formation by lowering the mitochondrial membrane potential by increasing the leak of protons from the intermembrane space back into the matrix, a process that also occurs to some extent through the ANT (22). The end result of this mild uncoupling of oxidative phosphorylation from ATP synthesis is a decrease in electron leak in the ETC, which attenuates ROS production (301).

It is unclear precisely how UCPs modulate proton leak; however, experimental evidence implicates fatty acids in the transport of protons by these inner mitochondrial membrane proteins (23). These processes have been most widely studied for the UCP1. One theory suggests an indirect role of UCPs in mediating proton transport (147). In this model, fatty acids are the carriers of protons from the intermembrane space to the matrix: protonated fatty acids diffuse across the inner mitochondrial membrane. After the release of protons from the fatty acids to the matrix, UCP1 transports the fatty acid anion to the intermembrane space (147). Another theory suggests that fatty acids directly associate with UCP1, and then facilitate proton transport across the membrane (217). Overall, studies suggest that fatty acid concentrations may be important in activating proton transport and may also regulate the expression and function of UCP2 and UCP3, as well as UCP1 (68).

Recent studies suggest that UCP2 and UCP3 may be modulated by S-glutathiolation, which maintains the carrier in an inactive state. In response to hydrogen peroxide, GR promotes the removal of -SG to activate UCP function (271). Further analysis is needed to understand fully the significance of this regulation, as these UCPs are also upregulated by transcriptional and translational mechanisms and have a short half-life in many tissues (22). Several studies have found an importance of these UCPs in nutrient regulation, fatty acid metabolism, and glucose-mediated insulin release. Modulation of UCPs has been considered as a strategy to prevent damage during ischemia/reperfusion injury in various models of cardiac and neuronal injury, as upregulation of proton leak due to UCP- or ANT-mediated mechanisms has been found to contribute to ischemic preconditioning in the heart $(285,307)$. In addition, overexpression of UCP2 or UCP1 has been found to be protective against hypoxia/reoxygenation death in isolated cells or in whole heart models of ischemia $(41,417)$; however, other studies implicate the metabolic changes (lower ATP and acidosis) caused by UCP2 overexpression with reduced survival in cardiomyocytes (43). Consistent with these latter findings, knockout of UCP2 was found to be protective in a model of cerebral ischemic injury (110). Some of this protection, however, may not be due to the lack of UCP2; rather, protection from oxidative injury may result from the compensatory upregulation of SOD2 and preservation of GSH in UCP2 knockout mice after ischemia compared to the wild-type mice.

Other studies, however, suggest that UCP2 helps to preserve dopamine neurons in a mouse model of Parkinson disease due, in part, to its ability to reduce ROS production: in this model deficient mice had more ROS and increased cell loss compared to wild-type controls, whereas UCP2 overexpressing mice were more protected with less ROS and less cell death (12). Possibly, differences in levels of expression and disease models account for some of these apparently contradictory findings regarding the beneficial role of UCP2. Overall, it seems that chronic changes in the expression of UCPs (or use of pharmacological uncouplers) may not provide a beneficial effect in all cases that involve excess production of oxidants, possibly due to long-term effects on cellular bioenergetics and substrate utilization that need 
further exploration. UCP2 may modulate insulin sensitivity, in part, by regulating oxidants in pancreatic $\beta$-cells, although there is contradictory evidence as to the beneficial role of $\mathrm{UCP} 2$ in mediating these processes. Early reports suggested the utility of decreasing UCP2 expression to enhance glucosemediated insulin release from pancreatic $\beta$-cells (473); however, subsequent findings suggest that excess oxidants generated by lack of UCP2 may promote pancreatic islet dysfunction, at least in some congenic strains of mice (328). These later studies propose that compensatory upregulation of antioxidant enzymes in the context of UCP2 deficiency decreases oxidative stress but also attenuates glucose-mediated insulin release. As discussed in other sections of this review, excess expression of antioxidant enzymes, such as GPx-1, can substantially reduce growth factor-mediated signaling due to the loss of essential ROS (169) and these alterations can contribute to insulin insensitivity (284). Studies in human populations support a role for $\mathrm{UCP} 2$ in regulating metabolism and diabetes as variants of the UCP2 gene have been associated with obesity, altered metabolism, and diabetes (68). Similarly, variants of UCP3, which is expressed in skeletal muscle, are associated with abdominal obesity, a known risk factor for type 2 diabetes (367), and expression of UCP3 is suppressed in diabetic muscle (68).

\section{Redox Regulation of Mitochondrial Turnover}

As discussed above, we now understand mitochondria as a network of structures that are constantly undergoing fission and fusion in order to adapt to cellular metabolic demands. Each mitochondrion may contain a different number of mitochondrial genomes, and the content of cellular mitochondria differs according to cell type. Cellular mitochondrial content is also in flux due to responses to growth factors and nutrients that may promote the biogenesis of new mitochondria or the selective removal of damaged mitochondria during mitophagy, a process that may serve to avoid apoptosis. In this section, we highlight the redox-dependent mechanisms that contribute to these processes.

\section{A. Mitochondrial biogenesis}

1. Overview of biogenesis. Mitochondrial biogenesis is a complex process that involves the coordinated expression of genes from both nuclear and mitochondrial genomes, as well as replication of $\mathrm{mtDNA}$ (176). The compact mtDNA genome encodes 13 essential subunits of the oxidative phosphorylation complexes; the remainder of the oxidative phosphorylation components and other mitochondrial proteins are encoded in nuclear DNA. Coordinated upregulation of mitochondrial and nuclear genes is accomplished, in part, via PGC $-1 \alpha$ and to some extent PGC- $1 \beta(337,458)$. These coactivators activate the transcription factors NRF1 and NRF2 to increase the transcription of oxidative phosphorylation genes, antioxidant genes, and other mitochondrial-specific genes, including those involved in mtDNA transcription and replication, such as transcription factor A (TFAM) (458). These transcription factors regulate ETC function by binding to distinct DNA target sequences in overlapping sets of genes $(154,373)$. NRF1 may also coordinately upregulate genes that regulate cell cycle and proliferation (60), and NRF2, which has also been called GA-binding protein (GABP), uniquely targets the cytochrome oxidase subunit 4 promoter $(154,373)$. The coordinated upregulation of antioxidant genes along with ETC proteins also provides protection against cellular ROS $(398,427)$. Other nuclear factors, such as the estrogen-related receptors (ERR), ERR $\alpha, E R R \beta$, and $E R R \gamma$, may similarly induce the biogenic process in conjunction with PGC-1-coactivators (380). Changes in cellular AMPK, growth factor receptor-mediated signaling, $\mathrm{NO}^{\bullet}$ and cGC/cGMP signaling, or oxidant stress may all influence mitochondrial biogenesis, in part through of PGC-1-induced pathways. For example, in skeletal muscle cells, the increase in intracellular $\mathrm{Ca}^{2+}$ caused by muscle contraction leads to activation of calcium/calmodulin and CREB activation to promote $P G C-1 \alpha$ transcription, while the consumption of ATP during contraction causes accumulation of AMP, which activates AMPK. AMPK upregulates PGC- $1 \alpha$ by transcriptional pathways and also by directly phosphorylating PGC- $1 \alpha$ to release it from a repressor protein. Indirectly, release from the repressor increases PGC$1 \alpha$ accessibility to SIRT deacetylases, to enhance PGC- $1 \alpha$ activity. Also, indirectly, AMPK can promote the activation of other biogenic pathways, such as the activation of MEF-2 [see Ref. (294), for a review of biogenic signaling mechanisms]. In the next sections we focus on the role of redox regulation in biogenesis.

2. ROS and biogenesis. In several model systems, acute stimulation with exogenous oxidants was shown to activate mitochondrial biogenesis, as measured by increases in mitochondrial mass, mtDNA, and the expression of biogenic regulators, such as PGC- $1 \alpha$, NRF1, and TFAM $(237,238)$. In other models, acute injury with oxidants was found to lead to early mitochondrial dysfunction followed by recovery of mitochondrial function caused by subsequent upregulation of PGC- $1 \alpha$. In renal proximal tubule cells, this PGC- $1 \alpha-$ dependent biogenic response was found to involve activation of p38-MAPK to promote EGF receptor (EGFR)-induced signaling (342). Although this study reported no effect of Aktmediated pathways downstream of EGFR in PGC- $1 \alpha$ activation, other studies have implicated PI3K/Akt-mediated pathways in the regulation of biogenic responses that are promoted by oxidants, such as tert-butylperoxide (330). One difference between these studies is that the latter used a cell model in which NRF1 was relatively overexpressed, possibly allowing for identification of Akt-mediated NRF1 phosphorylation and activation pathways that may not be essential for oxidant-induced biogenesis in all cell types. Redox inactivation of PTEN phosphatase that antagonizes PI3K signaling was proposed to play an essential role in this pathway of Akt-mediated biogenesis (330). It is well known that redoxinactivation of PTEN plays an important role in allowing phosphatidylinositol-3,4,5-triphosphate (PIP3) accumulation in the cell membrane to recruit PDK1 and Akt as part of the PDK1-mediated phosphorylation of Akt (269). In some cell types, Akt3, rather than Akt1, may play a role in growth factor-mediated stimulation of biogenic pathways (455), and other studies identify a role for Akt pathways in the heme oxygenase-mediated modulation of biogenesis, which involves oxidant activation of NRF1 transcription via antioxidant response elements in the NRF1 promoter (329).

One functional consequence of mitochondrial biogenesis following overexpression of PGC- $1 \alpha$ is the reduction of mitochondrial ROS and prevention of apoptosis in response to stressors such as high glucose (427). It is well known that mtDNA mutations, oxidative modifications of mitochondrial 
proteins, and deficiencies in functional mitochondrial complex subunits contribute to excess production of ROS in mitochondria. Thus, PGC- $1 \alpha$-mediated mitochondrial biogenesis may promote the replacement of dysfunctional mitochondria with increased electron leak, with those that perform more efficiently with less electron leak to reduce effectively mitochondrial ROS production. However, PGC-1 proteins may additionally decrease ROS due to the coordinate upregulation of antioxidant proteins downstream of PGC- $1 \alpha$. Thus, PGC- $1 \alpha$ upregulation is associated with increased expression of antioxidants, such as UCP2, Prx3, Prx5, Trx2, TrxR2, catalase, SOD2, and GPx-1, in various experimental models $(398,427)$. There has been keen interest in using PGC$1 \alpha$ to improve in vivo metabolic profiles in type 2 diabetes, for instance, as overexpression of PGC- $1 \alpha$ improves insulin sensitivity in cultured skeletal myocytes $(224,293)$. Contrary to predictions, however, transgenic PGC- $1 \alpha$ overexpression in skeletal muscle did not improve the in vivo metabolic profile in mice (87). Rather, although the skeletal muscle of these mice had increased mitochondria, they were also more sensitive to high-fat-induced insulin resistance. Thus, the effects of PGC$1 \alpha$ on other genes, such as those involved in fatty acid transport/utilization, may have increased fatty acid oxidation and intramuscular lipids to decrease insulin signaling. Alternatively, as mentioned above, PGC- $1 \alpha$-mediated upregulation of antioxidant genes may contribute to a loss of insulin-mediated signaling responses by decreasing necessary ROS flux under certain conditions. In other studies, electrotransfection of PGC- $1 \alpha$ constructs into rat skeletal muscle appeared to improve lipid metabolism and insulin signaling (37). It is unclear whether the lack of deleterious effects on metabolism in this latter study was due to the lower levels of overexpression achieved in the muscle or the relatively short time frame of overexpression compared to the transgene model. Furthermore, in the rat in vivo transfection model, fatty acid transporters were upregulated to some extent. Interestingly, PGC- $1 \alpha$ overexpression reduced triacylglycerol, diacylglycerol, and ceramide in muscle of treated obese Zucker rats, with some increases in levels of triacylglycerol in lean rats. Other treatments that more modestly increase PGC- $1 \alpha-$ expression also have biogenic effects that may be beneficial. Thus, drugs such as thiazolidinediones (TZDs), that are used to improve insulin sensitivity in diabetics by PPAR $\gamma$-mediated mechanisms, were found to improve mitochondrial function in a hyperglycemic model through a PGC-1-dependent mechanism that increases mitochondrial content, reduces ROS generation, and upregulates antioxidant enzymes (142). Some TZDs, however, have been associated with an increased risk of myocardial infarction (315), possibly caused by other adverse metabolic actions of these drugs. Drugs that stimulate AMPK may also activate these biogenic pathways to lower mitochondrial dysfunction and ROS production (228), suggesting another mechanism by which these agents may improve cellular metabolism and reduce oxidative damage.

3. Telomere dysfunction and mitochondrial biogenesis. Aging and diseases of aging, such as diabetes and cardiomyopathy, are associated with decreased mitochondrial function. Telomeres, the nucleoprotein complexes at the ends of chromosomes that serve to protect the ends of the chromosome, also undergo an age-related decrease in length that is linked to cellular senescence (322). The telomerase holoen- zyme, which maintains telomeres, includes an RNA subunit and a telomerase reverse transcriptase (TERT). Recent findings correlate deficiency of TERT (and subsequent loss of telomeres) with deficiencies in mitochondrial function attributed to decreases in PGC- $1 \alpha$ and PGC- $1 \beta$ expression and suppressed mitochondrial biogenesis (364). In this study, concurrent with decreased cellular mitochondrial content, cellular respiration was suppressed and ROS production increased. Furthermore, mice deficient in telomeres developed cardiomyopathy and had altered metabolism (364). One role of telomeres is to suppress p53 activation (84). p53 activation was found to suppress expression of the PGC- $1 \alpha$ and PGC- $1 \beta$ (364), thus linking telomeres to mitochondrial biogenesis. Other studies similarly report that overexpression of TERT can decrease ROS production and improve mitochondrial function (185), possibly via mitochondrial biogenesis. Additionally, increased expression of TERT may also improve antioxidant status by stimulating PGC- $1 \alpha$ and PGC $-1 \beta$, which are known to promote transcriptional upregulation of many antioxidant genes. TERT has also been found within mitochondria, although its role here is unclear. There have been reports that its mitochondrial localization can promote apoptosis, whereas other reports suggest that mitochondrial TERT may improve mitochondrial function or attenuate apoptotic activation (322). Although these noncanonical effects of TERT are not well understood, its translocation out of the nucleus following ROS-mediated phosphorylation by srckinases has been associated with apoptosis.

\section{B. ROS and mitophagy versus apoptosis}

Autophagy is the intracellular removal of cellular macromolecules and organelles through a catabolic process involving lysosomal degradation (469). An initial step in this process is the formation of a double-membrane isolation membrane around the cellular components, called an autophagosome, that later fuses with the lysomal compartment. This process can occur nonselectively during nutrient deprivation as a means of providing molecular sources of energy and building blocks for cell survival, or it can also occur in a more directed fashion to remove damaged or excess organelles. Some of the autophagy component proteins involved in starvation-induced autophagy, such as the Atg 4 family of enzymes, are regulated at redox-active Cys (375). It has been suggested that increased ROS production in starvation provides a necessary control mechanism in coordinating the activity of Atg4 during autophagy.

The selective engulfment of mitochondria and removal of mitochondria is referred to as mitophagy. Mitophagy can remove mitochondria damaged by oxidants and those producing excess oxidants. Recognition and removal of these mitochondria by mitophagy can avoid apoptotic changes by preventing the release of mitochondrial apoptogens, such as cytochrome c or AIF (242). Some of the specific components involved in mitophagy include the outer mitochondrial membrane protein PTEN-induced kinase 1 (PINK1) and parkin, an E3 ubiquitin ligase. Mutations and/or dysregulation of these genes have been implicated in neurodegeneration found in Parkinson disease $(148,426)$. Also crucial to these processes is mitochondrial fission. In damaged mitochondria, accumulated PINK1 fosters the translocation of parkin to mitochondria, the subsequent ubiquitination of 
mitochondrial proteins, and engulfment of mitochondria in autophagosomes $(311,437,469)$. Interestingly, complete loss of PINK1 can promote mitochondrial oxidant production and subsequent mitophagy (107), possibly due to mitochondrial membrane damage and loss of mitochondrial potential, which may promote the association of parkin with mitochondria (311). Importantly, mitophagy serves a protective function; loss of essential mitophagy pathways is thought to promote mitochondrial dysfunction and cell death.

\section{Heme oxygenase, carbon monoxide, and mitochondrial function}

Heme oxygenase-1 (HO-1) is rapidly induced by inflammatory cytokines. It catalyzes the degradation of heme to carbon monoxide (CO), biliverdin, and ferrous iron (467), and contributes to cellular protection against damage caused by ischemia-reperfusion injury (467), hypoxia (92), inflammatory cytokines (240), and other cellular insults. The mechanisms by which HO-1 promotes protection are not entirely clear, but some of these effects appear to be mediated via the production of $\mathrm{CO}$ and its heme-binding properties (272). Activation of the HO system, however, has also been associated with detrimental effects in diseases, such as Alzheimer disease or in aging, due, in part, to increases in mitochondrial iron accumulation and disruption in mitochondrial complex IV function, that correlate with increased HO-1 expression (376). One issue may be the duration and extent of $\mathrm{HO}-1$ expression and function. Thus, both $\mathrm{CO}$ and heme degradation productions have been shown to mediate protective and harmful effects, depending on the context of their production $(115,272)$. It is well known that excess $\mathrm{CO}$ is damaging due, in part, to its high affinity for heme moieties involved in oxygen transport and mitochondrial respiration. $\mathrm{CO}$, however, has also been shown to promote mitochondrial biogenesis and may in some instances be protective against mitochondrial dysfunction and cell death. In mice, brief $(1 \mathrm{~h})$ periods of exposure to $\mathrm{CO}$ were found to attenuate cardiac mitochondrial dysfunction, and lessen cardiac mitochondrial loss, oxidative stress, and apoptosis caused by doxorubicin (404). Doxorubicin, a chemotherapeutic agent, is well known to cause cardiomyopathy that contributes to comorbidity in cancer patients (409). Interestingly, exposure to $\mathrm{CO}$ in the doxorubicin studies correlated with increased HO-1 production in heart, suggesting that other heme degradation products could also contribute to the cardioprotective effects of inhaled CO. In cell culture studies, overexpression of HO-1 similarly attenuated doxorubicin-induced apoptosis and loss of mitochondrial content (404). These findings are consistent with other studies suggesting a role for HO-1 in upregulating mitochondrial biogenesis, by a pathway that implicates mitochondrial ROS as an essential contributor to biogenesis (329). Earlier studies by these and other investigators have shown that $\mathrm{CO}$ binds to cytochrome oxidase $a_{3}$ heme to increase mitochondrial leak of hydrogen peroxide $(470,475)$. Apparently, upregulation of these mitochondrial sources of ROS production are essential to promote both PGC-1 and NRF1 gene expression leading to increased mitochondrial biogenesis. This mechanism relies, in part, on ROS-mediated activation of Akt and its subsequent activation of NRF1 (329). This is another example of the signal-transduction role of moderate levels of excess ROS that serve to maintain (or reset, in this case) cellular homeostasis.
In vivo, overexpression of cardiomyocyte-targeted HO-1 improved survival after myocardial infarction by reducing myocyte-specific apoptosis and postinfarction left ventricular remodeling, in part, by preserving mitochondrial function (440). In particular, analysis of isolated cardiomyocytes and mitochondria from HO-1-overexpressing and control mice indicated that excess $\mathrm{HO}-1$ decreased mitochondrial respiration and attenuated $\mathrm{Ca}^{2+}$ - or phenylarsine oxide-induced mitochondrial swelling and mitochondrial membrane permeability transition. Furthermore, in this study, use of the $\mathrm{CO}$ donor tricarbonylchloro(glycinato)ruthenium (II) similarly improved mitochondrial function and protected the heart from left ventricular remodeling and apoptosis following infarct in an in vivo model of heart failure (440). Unlike the previous studies, this later study did not examine the effects of $\mathrm{HO}-1$ overexpression or $\mathrm{CO}$ donors on mitochondrial biogenesis; however, these findings are consistent with a mechanism involving $\mathrm{CO}$-induced protection of mitochondrial function. Another recent study (382) suggested that ROS induced by $\mathrm{CO}$ exposure regulate the function of an L-type $\mathrm{Ca}^{2+}$ channel by a redox mechanism to enhance cardioprotection. Thus, in isolated rat myocytes, exposure to $\mathrm{CO}$ or a $\mathrm{CO}$ donor causes reversible inhibition of L-type $\mathrm{Ca}^{2+}$ channels that could be replicated in HEK293 cells transfected with the $\alpha_{1 C}$ subunit of the human cardiac L-type $\mathrm{Ca}^{2+}$ channels containing a C-terminal splice variant. Apparently, COinduced ROS derived from mitochondria inhibit channel activity by a mechanism involving three Cys residues in the splice insertion region of this channel: mutation of any one of these Cys was sufficient to block CO's inhibitory effect. These studies do not show how CO-induced ROS affect the L-type $\mathrm{Ca}^{2+}$ channel, and only suggest Cys oxidation as a possible mechanism. In cardiomyocytes, however, $\mathrm{Ca}^{2+}$ overload can cause cell death, in part, via mechanisms involving uptake of excess $\mathrm{Ca}^{2+}$ into mitochondria, subsequent ROS production, and activation of the mitochondrial permeability pore transition (as discussed further in the next sections). Thus, an effect of $\mathrm{CO}$ on ROS and L-type $\mathrm{Ca}^{2+}$ channels could potentially modulate mitochondrial dysfunction, swelling, formation of the MPT pore, and apoptosis. Further analysis, however, is necessary to determine whether CO-mediated L-type $\mathrm{Ca}^{2+}$ channel regulation contributes to the in vivo effect of $\mathrm{CO}$ on cardiomyocytes.

\section{Redox Regulation of Apoptosis}

As discussed above, redox-sensitive thiols play a role in many aspects of apoptotic signaling. In this section we discuss redox mechanisms that effect apoptosis, analyzing some known pathways related to protein and phospholipid oxidation that drive apoptotic changes $(159,225)$.

\section{A. ASK1 and Trx-mediated regulation of JNK}

JNK1 and JNK2 are members of one of three major subfamilies of MAPK that mediate cell signaling in response to receptor and other stimuli. Each of these MAPK are activated by upstream kinases. The extracellular signal-related kinases, or ERK, are part of growth factor-mediated signal transduction scheme that promotes cell proliferation, differentiation, and survival pathways. In contrast, p38 MAPK and JNKs, which have collectively been referred to as stress-activated MAPK (SAPK), mediate the cellular responses to cytokines, 
oxidant stress, and other agents (230). Although the SAPKs can also mediate proliferative responses, prolonged activation of these SAPK can lead to apoptosis $(80,162)$. Oxidants downstream of cytokines and other stress activators are involved in JNK activation, as antioxidants can block JNK activation. This redox effect is due, in part, to regulation of the JNK activating kinase, apoptosis signal-regulated kinase 1 (ASK1) (Fig. 9). ASK1 is inhibited by Trx due to their proteinprotein interactions that maintain ASK1 in an inactive state (365). Trx redox state dictates, in part, ASK1 activity: Trx only binds to ASK1 when it is reduced, oxidized Trx dissociates from ASK1, leading to ASK1 activation (141). Once dissociated from Trx, ASK1 may also associate with other proteins such as TNF-receptor-associated adaptor proteins (TRAFs) in a signalosome that may be essential for ASK1 activation (282, 283). Downstream of ASK1, JNK can modulate nuclear factor targets to upregulate the transcription of pro-apoptotic factors and it can also migrate to the mitochondria. The absence of JNK was previously shown to limit $u v$ stress-induced mitochondrial release of cytochrome c (422), and other studies have shown that translocation of JNK to the mitochondria was essential for cytochrome c release and apoptosis downstream of other stress activators $(67,189)$. Furthermore, targeting of JNK to the mitochondria resulted in a significant increase in oxidants generated by complex I (67). One possible

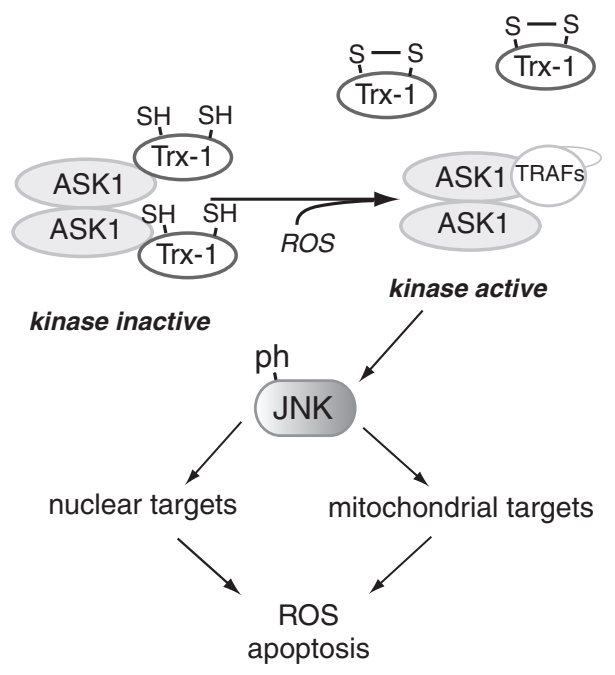

FIG. 9. Redox regulation of JNK activation and apoptotic pathways. JNK is one of the stress-activated protein kinases (SAPK) (230). Apoptosis signal-regulated kinase 1 (ASK1) is the mitogen-activated protein kinase (MAPK) kinase that regulates the SAPK response to oxidative stimuli. The redox regulation of ASK1 is indirect and due to protein-protein interactions with Trx1 (365). Reduced Trx1 association with ASK maintains ASK in its inactive form (141). Oxidation of Trx1 dissociates it from ASK, leading to the association of ASK with other proteins, including the tumor necrosis factorreceptor-associated adaptor proteins (TRAFs), which may promote ASK activation $(282,283)$. Activated ASK is free to target JNK, which, once phosphorylated, can promote a proapoptotic response. JNK phosphorylation of downstream nuclear factors can increase the expression of pro-apoptotic genes, and its phosphorylation of mitochondrial targets, such as $\mathrm{p} 66^{\text {shc }}$, can enhance mitochondrial ROS production and apoptogen release. mediator of JNK's effects on mitochondria is $\mathrm{p} 66^{\text {shc }}$, which apparently is a target of JNK $(235,250)$. Phosphorylation of p $66^{\text {shc }}$ may enhance its apoptotic effect in mitochondria (297). Prolonged JNK activation can also be caused by oxidative inactivation of DUSPs that dephosphorylate JNK (206). Thus, in some cell systems, increased expression of antioxidant proteins or other antioxidant treatments can lessen DUSP inactivation to reduce JNK activation and inhibit apoptosis.

\section{B. MPT pore and cell death}

1. Mitochondrial permeability transition. The MPT involves loss of inner mitochondrial membrane integrity due to the opening of a nonspecific pore (MPT pore) that allows for flux of small molecules, $<1500 \mathrm{Da}$, and protons, leading to mitochondrial swelling, loss of mitochondrial membrane potential, outer membrane rupture, and apoptotic or necrotic death. Formation of the pore can occur in response to many stimuli, including $\mathrm{Ca}^{2+}$ overload and oxidant stress, but MPT does not play a role in all forms of apoptosis $(46,125,309,438)$. In fact, some pathways lead to apoptosis and mitochondrial release of apoptogens in the absence of MPT induction.

The composition of the pore responsible for the enhanced inner membrane permeability is not clear. Implicated in MPT pore formation are the inner membrane ANT; the outer membrane VDAC; cyclophilin D, a matrix protein that is a peptidyl prolyl-cis, trans isomerase; and, possibly, the mitochondrial phosphate carrier (247). Although much experimental data exist for a role of ANT in the MPT (e.g., the ANT inhibitor bongkrekic acid, 20-carboxymethyl-6-methoxy-2,5, 17-trimethyldocosa-2,4,8, 10,14,18,20-hepataenedioic acid, blocks MPT), liver mitochondria that lack the major forms of ANT (ANT1 and ANT2) can still exhibit MPT depolarization (222), suggesting that ANT may be a modulator of MPT, rather than an essential component of the MPT pore [reviewed in Ref. (247)]. Similarly, absence of all known subtypes of VDAC does not impair MPT pore formation (26). Cyclosporin A, an inhibitor of cyclophilin D, is known to inhibit MPT responses to some stimuli. Further evidence for the involvement of cyclophilin D in MPT formation comes from studies with cyclophilin D knockout mice $(25,309)$. Consistent with a role of cyclophilin in some, but not all, pathways involving mitochondrial-dependent cell death, lack of cyclophilin D does not lessen cytochrome $\mathrm{c}$ release or apoptotic death in response to Bcl-2-mediated mechanisms, such as those activating Bax or tBid. Accordingly, some researchers consider that cyclophilin D-dependent (i.e., cyclosporin A-inhibitable) forms of MPT lead to necrosis rather than apoptosis. Nonetheless, cells from cyclophilin D-deficient mice show resistance to MPT and death from $\mathrm{Ca}^{2+}$ overload and oxidant stress. In addition, in the absence of cyclophilin D, mice are more protected from cardiac damage after ischemia/ reperfusion $(25,309)$; however, lack of cyclophilin D in cardiac cells may be detrimental under certain conditions, as this deficiency compromises $\mathrm{Ca}^{2+}$ handling in mitochondria, decreasing the ability of mitochondria to efflux $\mathrm{Ca}^{2+}$ after alterations in myocardial workload due to lack of beneficial (perhaps transient) MPT pore formation (123). Consequently, in cyclophilin D-depleted cells, faulty $\mathrm{Ca}^{2+}$ handling altered mitochondrial substrate utilization, decreasing fatty acid utilization in response to stressors (such as pressure overload) and promoting a reliance on glucose instead. This metabolic 
shift was found to contribute to cardiac hypertrophy and increased sensitivity to heart failure after pressure overload (123). A reliance on glucose rather than fatty acid metabolism is characteristic of heart failure in humans, as well (425). Interestingly, at very high levels of $\mathrm{Ca}^{2+}$, cyclophilin D-deficient cells or cells treated with cyclosporin A still have an MPT response $(25,31,167,309)$. These findings have led to the suggestion that other proteins, such as the phosphate carrier mentioned above, may be essential components of the MPT pore (247). Interestingly, in addition to cyclosporine A or bongkrekic, the MPT response can be blocked by polyamines, such as spermine or spermidine, possibly due to their antioxidant effects (5).

Both ANT and cyclophilin D may be targets for redox regulation. One study identified possible redox-active Cys in cyclophilin D. In this study, reducing and oxidizing conditions were used to study the role of each of the 4-Cys in cyclophilin D on its activity, intrinsic Trp fluorescence (a comparative measure of conformation), and ability to form intramolecular disulfides (258). Based on a series of experiments with single Ser for Cys substitutions in the protein, it was determined that two of these Cys, Cys203 and Cys157, may be involved in redox-mediated disulfide bond formation (258). These experiments, however, were mostly performed on isolated proteins and there is not a clear role for these reactive thiols within the mitochondria. Further study is needed to determine the functional significance of these thiols in cyclophilin D-mediated MPT pore opening. In contrast, the redox-sensitive thiols of ANT have been well studied as targets for modification by nitric oxide, peroxynitrite, thiol crosslinkers, and other thiol-reactive reagents $(101,435)$. ANT has three thiols that are implicated in its function that are located in three distinct hydrophilic loops $(102,167,171,286)$. ANT has two different conformational states depending on the location of the substrate binding site in the matrix (mstate) or cytosolic (c-state) side. Redox-sensitive disulfides form between Cys160 and Cys257, to enhance the " $\mathrm{c}$ " conformation of ANT, which is associated with diminished binding to ADP. The third thiol, Cys57, can be induced to form an intermolecular disulfide between ANT subunits only when it is in the " $\mathrm{m}$ " state but not the " $\mathrm{c}$ " state, confirming the proximity of these residues in the functional homodimer; however, these dimers also block transport function (171).

2. Mitochondrial $\mathrm{Ca}^{2+}$, redox, and mitochondrial dysfunction. As discussed above, overload of mitochondrial matrix $\mathrm{Ca}^{2+}$ can lead to MPT and cell death. Uniporters regulate mitochondrial matrix uptake of $\mathrm{Ca}^{2+}$ when its intracellular concentration is in the micromolar range (161). At nanomolar concentrations of intracellular $\mathrm{Ca}^{2+}$, its uptake into mitochondria has been proposed to be regulated by a $\mathrm{Ca}^{2+} / \mathrm{H}^{+}$exchanger that would favor import when mitochondria are hyperpolarized: the function of this exchanger is still highly speculative, but it may have bidirectional properties (370). The uptake of $\mathrm{Ca}^{2+}$ into mitochondria is blocked by ruthenium red, which actually blocks VDAC-mediated transport of $\mathrm{Ca}^{2+}$ into the intermembrane space (362). $\mathrm{Ca}^{2+}$ release from mitochondria is known to be driven by cellspecific $\mathrm{Na}^{+}$-dependent and $\mathrm{Na}^{+}$-independent pathways. In cardiomyocytes, for example, $\mathrm{Na}^{+}$-dependent pathways predominate. Mitochondrial uptake of $\mathrm{Ca}^{2+}$ is regulated, in part, by the release of intracellular stores from compartments such as the endoplasmic reticulum or the sarcoplasmic reticulum in muscle cells $(261,352)$. Mitochondrial uptake of $\mathrm{Ca}^{2+}$ can also buffer intracellular $\mathrm{Ca}^{2+}$ that enters cells from extracellular pools through $\mathrm{Ca}^{2+}$ channels. In contracting cardiomyocytes, normal release of $\mathrm{Ca}^{2+}$ from intracellular stores allows for uptake by mitochondria and the stimulation of $\mathrm{Ca}^{2+}$-sensitive enzymes in the TCA cycle $(\mathrm{PDH}, 2-$ oxoglutarate dehydrogenase, and $\mathrm{NAD}^{+}$-linked ICDHs) to coordinate energy production and demand, increasing NADH production and mitochondrial respiration (261). In other cells, mitochondrial buffering of $\mathrm{Ca}^{2+}$-channel uptake can maintain $\mathrm{Ca}^{2+}$ channel activity by decreasing local concentrations of $\mathrm{Ca}^{2+}$. Alternatively, $\mathrm{Ca}^{2+}$ uptake by mitochondria can serve to regulate intracellular $\mathrm{Ca}^{2+}$ transport (370). Excess $\mathrm{Ca}^{2+}$, however, can cause oxidative stress, in part, by its effects on mitochondrial phospholipid disposition and oxidation. In particular, $\mathrm{Ca}^{2+}$ affects cardiolipin, a membrane phospholipid that is found primarily in mitochondria. The role of oxidized phospholipids, including cardiolipin, in apoptosis is discussed further in the next section; however, it is important to mention briefly here the role of cardiolipin in $\mathrm{Ca}^{2+}$-mediated mitochondrial ROS production and MPT activation.

Both $\mathrm{Ca}^{2+}$ overload and cardiolipin oxidation enhance mitochondrial ROS production (321). The mechanisms by which $\mathrm{Ca}^{2+}$ promotes excess ROS generation are not well understood: it is thought that excess $\mathrm{Ca}^{2+}$ enhances ROS production, in part, due to its effects on TCA cycle enzymes, but possibly, the association of $\mathrm{Ca}^{2+}$ with cardiolipin contributes to ROS generation by disrupting the flow of electrons in complex III. One consequence of mitochondrial $\mathrm{Ca}^{2+}$ overload is the formation of the MPT pore. Antioxidant treatments have been found to attenuate $\mathrm{Ca}^{2+}$-induced MPT, whereas coexposure to oxidants augments MPT pore formation $(64,225,268)$. As discussed above, transient MPT pore formation may be protective; however, prolonged MPT pore opening is associated with the release of apoptogens to promote apoptotic cell death.

3. STAT3 and mitochondrial function. STAT3, initially characterized as a nuclear factor that regulates gene expression in response to IL-6 and other cytokines, has been recently found to have a specific role in modulating mitochondrial function through mechanisms independent of its function as a nuclear factor, but dependent on its phosphorylation at a serine residue $(412,445)$. The molecular mechanism by which STAT3 modulates ETC activity is not well understood, but deficiency of STAT3 has been shown to decrease complex I and complex II activity and ATP production while increasing mitochondrial ROS at complex I (412). Interestingly, these changes do not involve alterations in the expression of the mitochondrial subunits. Instead, it has been proposed that a direct interaction between mitochondrial STAT3 and complex I mediates the effects of STAT3 on mitochondrial respiration (412). Excess mitochondrially targeted STAT3 increases electron transport and oxidative phosphorylation, while decreasing ROS to protect against MPT pore formation in response to $\mathrm{Ca}^{2+}$ overload. Thus, cardiac-specific deletion of STAT3 in mice result in cardiac inflammatory fibrosis, dilated cardiomyopathy, and heart failure, and lack of STAT3 eliminates the protective effects of ischemic preconditioning (173). Confirming a role of STAT3 in cardioprotection, studies have 
found that overexpression of STAT3 in cardiomyocytes protects mice against doxorubicin toxicity, which is known to involve mitochondrial dysfunction. To study specifically the mitochondrial function of STAT3, independent of its function as a nuclear factor, a recombinant form of STAT3 that targets to the mitochondria and lacks the DNA binding domain was engineered (MLS-STAT3E). Expression of this mutant form of STAT3 to cardiomyocytes was found to protect hearts from ischemic damage by decreasing mitochondrial generation of ROS and attenuating cytochrome $\mathrm{c}$ release in a stop flow model of ischemia (411). Similar to STAT3-deficient mitochondria, overexpression of MLS-STAT3E decreased complex I and complex II activities; however, unlike the STAT3 deficiency, the MLS-STAT3E did not increase ROS generation at complex I. The reason for diminished complex I and II activities caused by overexpression of the DNA-binding domain-deficient construct is not clear, nor are the means by which endogenous STAT3 modulates mitochondrial function. These intriguing findings suggest the need for further analysis of the role of proteins that modify mitochondrial electron transport.

\section{Lipid oxidation and apoptotic signaling}

1. Cardiolipin oxidation and apoptosis. Cardiolipin is a membrane phospholipid found primarily in mitochondrial membranes. It has a unique structure with four fatty acyl side chains arranged in a symmetrical fashion on 2 (joined) diacyl phosposphatidyl acids. In the heart, cardiolipin is noted to have a high content of linoleic acid that is essential for its ability to promote ETC complex III and IV activity (396). Cardiolipin also fosters the formation of supercomplexes within the inner mitochondrial membrane that have enhanced activity $(327,353)$. The presence of cardiolipin within the inner mitochondrial membrane may also modulate the activity of other resident proteins, such as ANT (96). Cardiolipin is thought to play an essential role in maintaining cytochrome $\mathrm{c}$ in the inner membrane where it constitutes nearly $20 \%$ of membrane lipids. Oxidation of cardiolipin disrupts its interaction with cytochrome $c$ to foster the release of cytochrome c from mitochondria, which promotes apoptosis. Compared to the other major phospholipids of mitochondria, phosphatidylethanolamine and phosphatidylcholine, cardiolipin was found to be more sensitive to oxidative stress (451). Interestingly, cardiolipin may be oxidized, in part, through the peroxidase activity of cytochrome $\mathrm{c}$ in response to increased production of hydrogen peroxide after disruption in electron transport $(34,310)$. It has been proposed that cytochrome c association with cardiolipin is key to its transition from an electron acceptor/donor in ETC to a peroxidase (30, 204). Cytochrome c only functions as a peroxidase in association with cardiolipin, resulting in the oxidation of cardiolipin, which subsequently potentiates cytochrome c release from the mitochondria. Once in the cytosol, cytochrome c promotes the formation of the apoptosome, which involves the oligomerization of apoptotic releasing factor-1 (Apa-f1). Apoptosome recruitment of caspase 9, an initiator caspase, begins the cascade that activates caspase 3, leading to apoptotic cell death (251).

The preservation of cardiolipin helps to maintain mitochondrial function and prevent apoptosis. Thus, there is an interest in targeting cardiolipin/cytochrome $\mathrm{c}$ interactions to modulate cell survival or apoptosis. Melatonin, a lipophylic hormone that has been shown to have properties as a free radical scavenger (9), was found to reduce lipid peroxidation in cardiac mitochondria after ischemia/reperfusion injury while preserving complex I and III activity (326). In addition, in vitro studies on isolated mitochondria indicated that melatonin had a similar protective effect on complex I and III function and preserved mitochondrial cardiolipin content, suggesting that melatonin protected mitochondria directly, perhaps via an antioxidant effect rather than an indirect effect activating other protective cellular mechanisms (326). Similarly, in studies of intact hearts, inhibition of complex I (with rotenone) during ischemia preserved mitochondrial cardiolipin and cytochrome c content (246), suggesting a role for ETC leak in cardiolipin oxidation and a possible target to prevent cardiolipin oxidation. Other therapeutic means to regulate ETC leak are being developed, including S-nitrosating compounds, that were discussed previously $(306,336)$, and cationic plastoquinone derivatives (392). The function of GPx-4 to limit specifically mitochondrial phospholipids oxidation has also been explored, as discussed next.

2. Membrane phospholipids and AIF-mediated cell death. Of the GPxs, GPx-4 is somewhat unique because it will reduce membrane phospholipid hydroperoxides, as well as soluble hydroperoxides. Recent findings, however, suggest that GPx-4 primarily targets oxidized membrane phospholipids (383). A role for GPx-4 in regulating cardiolipin oxidation in particular has not been examined, but one of the isoforms of GPx-4 targets to the mitochondria, where possibly it may also serve to reduce mitochondrial phospholipids. In fact, overexpression of mitochondrial targeted GPx-4, but not the nonmitochondrial form, suppressed apoptosis, and reduced mitochondrial hydroperoxide content $(316,340)$ in response to a variety of exogenous agents, including 2deoxyglucose, etoposide, $t$-butylhydroperoxide, and diquat. Complete absence of GPx-4 is known to be lethal (465), and studies show that deficiency of GPx-4 promotes cellular apoptosis via the accumulation of lipid peroxidation in the absence of exogenous oxidants or stimuli (383). Interestingly, a deficiency of GPx-4 appears to potentiate 12/15-lipoxygenasemediated lipid peroxidation to increase cell death via the release of AIF, an apoptogen from mitochondria (383). Consistent with previous reports (407), AIF migration from the mitochondria to nucleus was found to mediate apoptosis in a manner that could not be blocked by overexpression of the antiapoptotic Bcl-2 or by caspase inhibition. The absence of GPx-4 was suggested to play a role in the activation of 12/ 15-lipoxygenase and to promote lipid peroxidation. Lipid peroxidation and cell death could also be potentiated in GPx-4replete cells by inhibiting GSH synthesis. This finding is consistent with the role of GPx-4 in modulating lipoxygenase activity (378). The role of lipid peroxides in promoting apoptosis was proven by the finding that the lipid-soluble $\alpha$ tocopherol could prevent apoptosis, whereas $\mathrm{N}$-acetyl Cys or other water-soluble antioxidants could not block cell death in GPx-4-deficient cells (383). In models of GPx-4 overexpression, excess GPx-4 also blocked cytochrome c release from mitochondria and caspase activation in models where oxidative stress was induced by exogenous agents $(316,340)$, although it did not prevent apoptosis from FasL (extrinsic) pathway activation. Taken together, these findings suggest a crucial role 
for antioxidant mechanisms in preserving mitochondrial membrane redox potential to prevent apoptosis.

\section{Mitochondrial Responses to Hypoxia}

HIF family transcription factors are master regulators of hypoxic responses. These factors are heterodimers that consist of a common $\beta$ chain that is constitutively expressed and not responsive to oxygen levels, and one of three $\alpha$ chains (HIF- $1 \alpha$, HIF- $2 \alpha$, and HIF- $3 \alpha$ ), which are primarily regulated at the level of protein stability, according to oxygen tension. Of these HIF- $1 \alpha$ is most well studied for its role in modulating metabolic responses to hypoxia in most tissues. HIF- $2 \alpha$ may upregulate some unique targets, such as erythropoietin, and, in some cells, may also contribute to the hypoxia-mediated increase in other genes, like vascular endothelial growth factor or plasminogen activator inhibitor-1 (343). Here, we will focus on HIF- $1 \alpha$, due to its effects on mitochondrial respiration and ROS (Fig. 10). Under normal oxygen tension, HIF- $1 \alpha$ is targeted for proteasomal degradation through the concerted action of prolyl hydroxylase (PHD) enzymes that modify two conserved prolines in the HIF- $1 \alpha$ protein $(279,397)$. PHD are $\mathrm{O}_{2}$-dependent enzymes that utilize ferrous iron and $\alpha$ ketoglutarate $(16,320)$. PHDs are inactive at low $\mathrm{O}_{2}$ and can be inactivated by the accumulation of TCA metabolites fumarate

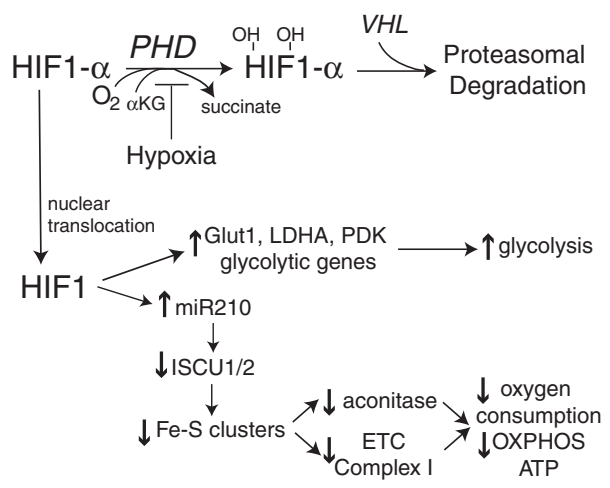

FIG. 10. Oxygen tension, hypoxia-inducible factor-1 (HIF1) activation of $\mathrm{miR} 210$ and mitochondrial function. Under normal $\mathrm{O}_{2}$ tension, HIF- $1 \alpha$ is hydroxylated by the prolyl hydroxylases (PHD), which utilize $\mathrm{O}_{2}$ and $\alpha$-ketoglutarate $(\alpha \mathrm{KG})$ as cosubstrates (203). Hydroxylated HIF- $1 \alpha$ is targeted for ubiquitination after recognition by the von HippelLindau tumor suppressor (VHL) protein, leading to its subsequent proteasomal degradation. Under hypoxia, decreased $\mathrm{O}_{2}$ tension suppresses PHD activity, allowing for HIF- $1 \alpha$ translocation to the nucleus, where it combines with HIF- $\beta$, which is not sensitive to $\mathrm{O}_{2}$ tension, to form the transcriptionally active dimer HIF-1. HIF-1 upregulates gene expression of the glucose transporter-1 (Glut1), LDHA, PDK, and glycolytic genes to promote an increase in glycolysis (385, 386). HIF-1 also transcriptionally activates the expression of miR210, which inhibits translation of iron-sulfur (Fe-S) cluster assembly protein isoforms ISCU1 and ISCU2 (69). A loss of Fe-S cluster formation results in a suppression of ETC complexes, including complex I and aconitase, which rely on Fe-S prosthetic groups for their function. The consequences of diminished Fe-S cluster formation suppress ROS in hypoxia, reduce oxygen consumption, decrease respiration, and decrease oxidative phosphorylation (Pasteur effect) (69). and succinate, which may occur from mutations in the tumor suppressor genes succinate dehydrogenase subunit B or fumarate hydratase $(187,334,384)$. [Succinate is a product of the PHD hydroxylation reaction; other TCA metabolites, such as fumarate as well as oxaloacetate, have been found to inhibit PHDs (221).] Prolyl hydroxylation allows for the interactions of HIF-1 $\alpha$ with von Hippel-Lindau tumor suppressor (VHL), which fosters the ubiquitination and degradation of prolyl hydroxylated-HIF-1 $\alpha$ (203). Thus, under hypoxia, prolyl hydroxylation is inhibited, and HIF- $1 \alpha$ escapes degradation, accumulates in the nucleus, and initiates gene transcription. Alterations in normal PHD/VHL/HIF-mediated pathways is thought to contribute to the metabolic adaptation found in Warburg tumors, that is, those that manifest enhanced glycolysis in the presence of $\mathrm{O}_{2}(35,444)$.

There is an ongoing debate as to the role of mitochondrial oxidants in regulating HIF-1 activation and the hypoxic response (72). Experimental evidence implicates a hypoxiainduced burst of mitochondrial ROS as necessary for HIF- $1 \alpha$ and HIF- $2 \alpha$ stabilization, as cells deficient in mitochondrial ROS, due to genetic or pharmacological treatments, have decreased HIF activation (53, 164, 274). One possible explanation for the potentiating effect of an ROS burst on HIF- $\alpha$ signaling is that an oxidant burst may promote oxidation of the $\mathrm{Fe}^{2+}$ required for the function of PHD enzymes, thereby reducing HIF- $\alpha$ degradation (202). Alternatively, these enzymes also are inhibited by succinate, and fluctuations in the TCA cycle could similarly diminish their ability to hydroxylate HIF- $\alpha$. Interestingly, lack of mitochondrial ROS does not prevent activation of HIF- $\alpha$ subunits in anoxia, thus suggesting that some oxygen is necessary for mitochondria to promote HIF- $\alpha$ signaling. Although mitochondrial complex III has been implicated in the oxidant burst by mitochondria, to date, the mechanism by which reduced oxygen tension mediates ROS generation is not well understood. Nonetheless, experimental evidence suggests that enhanced ROS production is not a sustained event in hypoxia $(69,385)$.

HIF-1 coordinately upregulates many genes to regulate metabolism, energy production, and mitochondrial function (385). Thus, under hypoxia, cells switch to oxygen-independent glycolysis (the Pasteur effect) through activation of HIFmediated transcriptional responses. HIF-1 coordinately upregulates PDH kinase, LDHA, and other glycolytic genes to increase glycolysis; decreases the activity of the TCA cycle; and reduces electron flux through mitochondrial complexes I, III, and IV $(214,386)$. To compensate for the increased reliance on glycolysis, HIF-1 also upregulates the expression of the Glut1, a glucose transporter. This transcription factor also promotes a subunit switch in cytochrome c oxidase 4 or COX 4 from 4-1 to 4-2 during hypoxia, which improves the efficiency of electron transport at complex IV (143). The result of these expression changes optimizes the control of energy production and minimizes the generation of potentially toxic ROS from increasingly inefficient mitochondrial electron transport under conditions of hypoxia.

Our recent findings suggest yet another mechanism by which HIF- $1 \alpha$ modulates electron transport and metabolism by ultimately suppressing the formation of Fe-S clusters, which are integral to the function of the respiratory chain. This HIF- $1 \alpha$-mediated response relies specifically on upregulation of a microRNA, miR210, that decreases the expression of ironsulfur cluster assembly proteins (ISCU1/2) by inhibiting their 
translation. Subsequent studies by other investigators confirm the role of miR210 in suppressing ISCU1/2 and as well as other factors (cytochrome c oxidase assembly protein) essential for ETC assembly $(81,129)$. ISCU1/2 arise from splice variants of the same transcript. ISCU1 protein is found in the cytosol, whereas ISCU2 targets to the mitochondria (420); we refer to them as ISCU1/2 here. These proteins facilitate the assembly of [4Fe-4S] and [2Fe-2S] Fe-S clusters, which are essential for the function of some apoproteins. These Fe-S prosthetic groups promote electron transport and redox reactions integral to many cellular processes ranging from electron transport and oxidative phosphorylation, ribosome biogenesis, purine catabolism, heme biosynthesis, DNA repair, to iron metabolism, among others; mutations in ISCU have been associated with a metabolic myopathy (360). Fe-S clusters are found in key mitochondrial respiratory complexes (complexes I, II, and III) where they facilitate electron transport (360) and in aconitase, a TCA enzyme.

We found that the miR210-induced decrease in ISCU1/2 has functional consequences that directly alter the metabolic phenotype of endothelial cells by decreasing complex I function, mitochondrial respiration, and ATP synthesis (69). Similarly, in other studies, miR210-mediated suppression of ETC and TCA enzymes were found to correlate with enhanced anaerobic glycolysis and increases in lactate production $(81,129)$. In our studies, we found that we could restore the ETC and TCA enzyme activities in the presence of elevated miR210 by using ISCU1/2 gene constructs that lack the miR210 recognition sequence in their $3^{\prime}$ untranslated region, suggesting that these miR210-induced deficiencies were primarily due to lack of Fe-S clusters. We also established a link between ICSU1/2 and suppression of miR210 expression by showing that siRNA knockdown of miR210 during hypoxia augments ROS production, illustrating a role for miR210 in modulating compensatory mechanisms to protect cells from ROS during hypoxia. Interestingly, during normoxia, forced expression of miR210 caused an increase in ROS generation $(69,81)$, possibly due to deficiencies in complex I that may promote electron leak in the presence of molecular oxygen. We suggest, however, that miR210-mediated inhibition of Fe$S$ clusters promotes cell survival, in part, by its suppression of mitochondrial respiration; possibly, miR210 upregulation may also promote cell survival by lessening ROS generation during the hypoxia to normoxia transition (Pasteur effect). miR210-enhanced cell survival, however, may not always be beneficial. Thus, in cancer or in pulmonary hypertension, metabolic shifts promoted by HIF and miR210 may increase cell growth and proliferation to contribute to tumor survival or vascular remodeling, respectively $(122,131,390)$. In addition, in a chronic setting, suppression of Fe-S cluster formation may have consequences for the synthesis of heme, the uptake and intracellular metabolism of iron, and genomic stability $(430,466)$. Forced expression of miR210 in cancer cell lines slowed initiation of tumor growth after implantation of these cells in nude mice; however, miR210 overexpression did not appear to increase cell death (179). Thus, these findings may suggest in the absence of other HIF-regulated genes miR210 may not produce the same results as when it is upregulated downstream of HIF. Other considerations that need further study are the additional targets that may be suppressed by miR210 in hypoxia, which may contribute to the adaptive responses to hypoxia.
In addition, the hypoxia-induced suppression of Fe-S protein activity can be contrasted with the suppression of Fe-S protein activity found in Friedreich's ataxia, a hereditary neurogenerative disease that results from decreased production/function of the frataxin protein $(17,220)$. Frataxin is a mitochondrial iron chaperone; its precise function is still a matter of debate but its deficiency leads to an increase in mitochondrial iron, mitochondrial dysfunction, and decreased function of Fe-S cluster containing proteins, including complex I and complex III, succinate dehydrogenase (which is both complex II in the ETC and part of the TCA cycle), and aconitase. Decreased expression of frataxin also increases the Fenton reaction due to excess free iron $(17,220)$. Studies indicate that loss of normal frataxin leads to excess mitochondrial production of hydrogen peroxide. Increased production of mitochondrial oxidants was found to correlate with a decrease in both total GSH as well as a decrease in the GSH/GSSG ratio in cells with frataxin deficiency; these changes may lead to the increased accumulation of protein Sglutathiolation in this disease (17). Interestingly, as mentioned above, forced miR210 overexpression under normoxic conditions is also associated with excess production of cellular oxidants $(69,81)$, although less is known about the species produced and the consequence of this production on GSH redox. Cells with frataxin deficiency show an inability to upregulate mitochondrial MnSOD, leading to some controversy regarding the importance of MnSOD and superoxide in the disease phenotype $(17,220)$; however, other studies confirm a role for hydrogen peroxide rather than superoxide in the damaging effects of this deficiency. For example, in a Drosophila model of Friedreich's ataxia, superoxide scavengers had no protective effect on the phenotype, whereas mitochondrial targeted catalase or Prx were protective (11).

\section{Conclusion}

Redox signaling allows for the rapid adaptation to a variety of environmental stimuli, such as hormones, nutrients, and $\mathrm{O}_{2}$ tension. The mitochondrion plays a central role in cellular adaptation: modulating metabolic, energetic, and ROS responses to promote cell growth and survival or cell death in response to a range of stimuli (Fig. 11). The overall redox status is kept in balance by a host of antioxidant enzyme systems, notably GPxs/GSH/GR/Grx and Prx/Trx/TrxR, that modulate cellular ROS flux and lipid oxidation. Grx/ GSH/GR and Trx/TrxR also reduce protein disulfides. Notably, each of these enzyme systems require NADPH to regenerate the reducing cosubstrate (GSH or Trx), highlighting the importance of G6PD to regenerate NADPH. In mitochondria, the NNT enzyme, which reduces $\mathrm{NADP}^{+}$utilizing the proton gradient and NADH from the TCA cycle, may also have a crucial role in maintaining mitochondrial NADPH.

Proteins offer unique redox switches that are reversibly modified by ROS to alter their function. Protein localization, thiol accessibility, $\mathrm{pK}_{\mathrm{a}}$, and protein-protein interactions may all contribute to the specificity of these responses. Mitochondrial function can be modulated directly by protein thiol oxidation or by lipid peroxidation (Fig. 12), and indirectly following the redox-dependent activation of phosphatases (Fig. 8), HIF (Fig. 10), ASK1 (Fig. 9), or other redox-sensitive proteins. Nutrient changes can also lead to $\mathrm{NAD}^{+}$ 


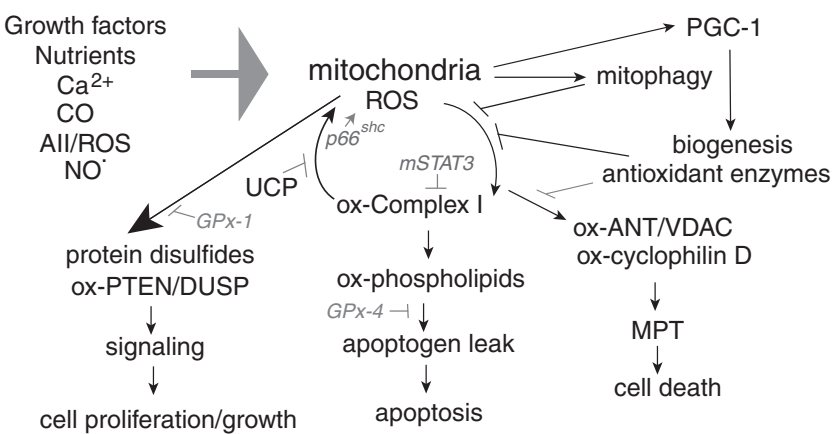

FIG. 11. ROS, redox, and mitochondria. Growth factors, nutrients, $\mathrm{Ca}^{2+}$ fluxes, ROS and its generators (including angiotensin II [AII] and carbon monoxide [CO]), and NO modulate mitochondrial function in a number of ways, including modulating respiration, promoting mitochondrial ROS production, or regulating cellular mitochondrial content by stimulating biogenic or mitophagy mediators. Cellular antioxidants and uncoupling proteins (UCP) modulate cellular responses to essential and excess cellular ROS. Mitochondria both generate and respond to these ROS. Essential ROS may be generated during normal respiration or in response to growth factor signaling, which stimulates ROS generation from mitochondria. Lack of this essential signaling can alter cellular redox balance, protein disulfide formation, and cell signaling via reversible thiol oxidation (for instance, of PTEN or DUSP phosphatases) or other mechanisms $(169,264,464)$. Antioxidants like GPx-1 may attenuate these responses. Essential ROS may also play a role in modulating mitochondrial biogenesis (350). AII receptor activation is one stimulus that may lead to the generation of excess ROS. Thus, AII-mediated stimulation of NOXdependent ROS was found to enhance mitochondrial production of ROS to promote mitochondrial dysfunction (121). Excess ROS can cause oxidative damage to members of the ETC, such as complex I, or members of the TCA cycle, such as aconitase or $\alpha$-ketoglutarate, or other mitochondrial proteins (4). Excess ROS can also promote the mitochondrial recruitment of proteins such as $\mathrm{p} 66^{\text {shc }}$ that further amplify ROS production, thereby contributing to cell death (ROSmediated ROS generation) (297). Other mitochondrial proteins, such as mitochondrial STAT3 (mSTAT3), appear to preserve ETC activity by preventing ROS leak at complex I. Excess ROS leads to redox imbalance, overoxidation of protein thiols, oxidation of mitochondrial lipids, and mitochondrial permeability transition (MPT). At some threshold of ROS, these changes result in necrosis or apoptosis. Cells possess several mechanisms to prevent excess ROS, including the biogenic program, which generates new mitochondria and increases the expression of some antioxidant genes (398), as well as mitophagy, which may target and remove damaged mitochondria (469). ROS upregulation of antioxidants or UCP may also modulate the responses of excess ROS (301), and, under some circumstances, brief MPT may alleviate mitochondrial stress to prevent mitochondrial dysfunction (123).

accumulation and activation of SIRT-dependent pathways that can include mitochondrial biogenesis (Fig. 6). Sites within the mitochondria can be regulated by SIRT deacetylation, or by direct oxidative modification. Complex I, for example, is a target for S-NO and other forms of thiol oxidation, which may inhibit its function, or possibly, promote electron leak (Fig. 7).

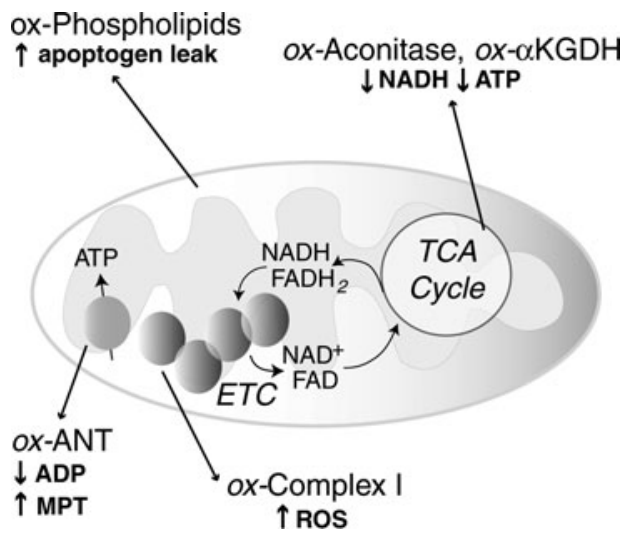

FIG. 12. Mitochondrial targets oxidatively modified by ROS. ROS can directly modulate the mitochondria by reacting with many redox-sensitive targets. Among these targets, membrane lipid peroxidation (ox-phospholipids) can lead to the release of apoptogens, such as cytochrome $\mathrm{c}$ and apoptosis-inducing factor, causing subsequent apoptosis. Antioxidant enzymes, such as GPx-4, which can reduce oxidized membrane phospholipids, attenuate these apoptotic modifications $(316,340)$. Enzymes in the TCA cycle, such as aconitase or $\alpha-\mathrm{KGDH}$, are susceptible to oxidative inactivation (ox-) (423). Decreased flux through the TCA cycle results in decreased NADH production, thereby reducing electron transport (ETC) and oxidative phosphorylation. Oxidation of the adenine nucleotide transporter (ANT) results in the formation of intramolecular disulfide bonds that alters the ability of the transporter to uptake ADP (101). This inactivation of the ANT sensitizes mitochondria to a permeability transition (MPT), leading to the loss of integrity of the mitochondrial membrane (potential), mitochondrial swelling, and cell death. Although other ETC complexes are also susceptible to oxidative modification, oxidation of complex I is associated with decreased complex I activity and increased production of superoxide $(120,416)$.

Other proteins, such as mitochondrial STAT3, may protect complex I from oxidative inactivation by mechanisms not well understood that involve preserving ETC function and decreasing ROS production after oxidative stress (Fig. 11). In contrast, oxidative mechanisms that activate $\mathrm{p} 66^{\text {shc }}$ to promote its mitochondrial localization can augment ROS generation, contributing to redox modifications to proteins and phospholipids. Downstream of ROS, other modifications of mitochondrial proteins can lead to mitophagy or activation of the MTP. Mitophagy and biogenesis are seen as two complementary processes that help to maintain metabolic homeostasis and reduce ROS accumulation. Brief openings of MTP pore may serve a protective function in mitochondria, but more prolonged MTP pore formation can lead to cell death. Excess mitochondrial ROS can also lead to detrimental cardiolipin oxidation. Such alterations in phospholipid oxidation promote mitochondrial-dependent apoptosis. Downstream of HIF activation, inhibition of Fe-S complex formation reduces ETC and TCA cycle enzymes to decrease ROS generation in hypoxia. The examples discussed in this review provide some insights into mechanisms by which redox events regulate mitochondrial function to alter cellular energetics and metabolism. 


\section{Acknowledgments}

The authors wish to thank Stephanie Tribuna for her expert editorial assistance. This work was supported by grants HL61795, HL48743, HL70819, HL107192, and HL108630.

\section{References}

1. Abu-Soud HM, Yoho LL, and Stuehr DJ. Calmodulin controls neuronal nitric-oxide synthase by a dual mechanism. Activation of intra- and interdomain electron transfer. J Biol Chem 269: 32047-32050, 1994.

2. Adachi T, Pimentel DR, Heibeck T, Hou X, Lee YJ, Jiang B, Ido $\mathrm{Y}$, and Cohen RA. S-glutathiolation of Ras mediates redox-sensitive signaling by angiotensin II in vascular smooth muscle cells. J Biol Chem 279: 29857-29862, 2004.

3. Adimora NJ, Jones DP, and Kemp ML. A model of redox kinetics implicates the thiol proteome in cellular hydrogen peroxide responses. Antioxid Redox Signal 13: 731-743, 2010.

4. Ago T, Kuroda J, Pain J, Fu C, Li H, and Sadoshima J. Upregulation of Nox4 by hypertrophic stimuli promotes apoptosis and mitochondrial dysfunction in cardiac myocytes. Circ Res 106: 1253-1264, 2010.

5. Agostinelli E, Marques MP, Calheiros R, Gil FP, Tempera G, Viceconte N, Battaglia V, Grancara S, and Toninello A. Polyamines: fundamental characters in chemistry and biology. Amino Acids 38: 393-403, 2010.

6. Ahn BH, Kim HS, Song S, Lee IH, Liu J, Vassilopoulos A, Deng CX, and Finkel T. A role for the mitochondrial deacetylase Sirt3 in regulating energy homeostasis. Proc Natl Acad Sci U S A 105: 14447-14452, 2008.

7. Aikens J and Dix TA. Perhydroxyl radical (HOO.) initiated lipid peroxidation. The role of fatty acid hydroperoxides. J Biol Chem 266: 15091-15098, 1991.

8. Alderton WK, Cooper CE, and Knowles RG. Nitric oxide synthases: structure, function and inhibition. Biochem J 357: 593-615, 2001.

9. Allegra M, Reiter RJ, Tan DX, Gentile C, Tesoriere L, and Livrea MA. The chemistry of melatonin's interaction with reactive species. J Pineal Res 34: 1-10, 2003.

10. Allen S, Balabanidou V, Sideris DP, Lisowsky T, and Tokatlidis K. Erv1 mediates the Mia40-dependent protein import pathway and provides a functional link to the respiratory chain by shuttling electrons to cytochrome c. J Mol Biol 353: 937-944, 2005.

11. Anderson PR, Kirby K, Orr WC, Hilliker AJ, and Phillips JP. Hydrogen peroxide scavenging rescues frataxin deficiency in a Drosophila model of Friedreich's ataxia. Proc Natl Acad Sci U S A 105: 611-616, 2008.

12. Andrews ZB, Horvath B, Barnstable CJ, Elsworth J, Yang L, Beal MF, Roth RH, Matthews RT, and Horvath TL. Uncoupling protein-2 is critical for nigral dopamine cell survival in a mouse model of Parkinson's disease. J Neurosci 25: 184-191, 2005.

13. Aon MA, Cortassa S, Maack C, and O'Rourke B. Sequential opening of mitochondrial ion channels as a function of glutathione redox thiol status. J Biol Chem 282: 21889-21900, 2007.

14. Aon MA, Cortassa S, and O'Rourke B. Redox-optimized ROS balance: a unifying hypothesis. Biochim Biophys Acta 1797: 865-877, 2010.

15. Aquilano K, Baldelli S, Cardaci S, Rotilio G, and Ciriolo MR. Nitric oxide is the primary mediator of cytotoxicity induced by GSH depletion in neuronal cells. J Cell Sci 124: 1043-1054, 2011.
16. Aragones J, Schneider M, Van Geyte K, Fraisl P, Dresselaers T, Mazzone M, Dirkx R, Zacchigna S, Lemieux H, Jeoung NH, Lambrechts D, Bishop T, Lafuste P, Diez-Juan A, Harten SK, Van Noten P, De Bock K, Willam C, Tjwa M, Grosfeld A, Navet R, Moons L, Vandendriessche T, Deroose C, Wijeyekoon B, Nuyts J, Jordan B, Silasi-Mansat R, Lupu F, Dewerchin M, Pugh C, Salmon P, Mortelmans L, Gallez B, Gorus F, Buyse J, Sluse F, Harris RA, Gnaiger E, Hespel P, Van Hecke P, Schuit F, Van Veldhoven P, Ratcliffe P, Baes M, Maxwell P, and Carmeliet P. Deficiency or inhibition of oxygen sensor Phd1 induces hypoxia tolerance by reprogramming basal metabolism. Nat Genet 40: 170180, 2008.

17. Armstrong JS, Khdour O, and Hecht SM. Does oxidative stress contribute to the pathology of Friedreich's ataxia? A radical question. FASEB J 24: 2152-2163, 2010.

18. Arner ES. Focus on mammalian thioredoxin reductasesimportant selenoproteins with versatile functions. Biochim Biophys Acta 1790: 495-526, 2009.

19. Asahi M, Fujii J, Suzuki K, Seo HG, Kuzuya T, Hori M, Tada M, Fujii S, and Taniguchi N. Inactivation of glutathione peroxidase by nitric oxide. Implication for cytotoxicity. J Biol Chem 270: 21035-21039, 1995.

20. Asahi M, Fujii J, Takao T, Kuzuya T, Hori M, Shimonishi Y, and Taniguchi N. The oxidation of selenocysteine is involved in the inactivation of glutathione peroxidase by nitric oxide donor. J Biol Chem 272: 19152-19157, 1997.

21. Azad N, Vallyathan V, Wang L, Tantishaiyakul V, Stehlik C, Leonard SS, and Rojanasakul Y. S-nitrosylation of Bcl-2 inhibits its ubiquitin-proteasomal degradation. A novel antiapoptotic mechanism that suppresses apoptosis. J Biol Chem 281: 34124-34134, 2006.

22. Azzu V and Brand MD. The on-off switches of the mitochondrial uncoupling proteins. Trends Biochem Sci 35: 298307, 2010.

23. Azzu V, Jastroch M, Divakaruni AS, and Brand MD. The regulation and turnover of mitochondrial uncoupling proteins. Biochim Biophys Acta 1797: 785-791, 2010.

24. Bach D, Pich S, Soriano FX, Vega N, Baumgartner B, Oriola J, Daugaard JR, Lloberas J, Camps M, Zierath JR, Rabasa-Lhoret R, Wallberg-Henriksson $H$, Laville M, Palacin M, Vidal H, Rivera F, Brand M, and Zorzano A. Mitofusin-2 determines mitochondrial network architecture and mitochondrial metabolism. A novel regulatory mechanism altered in obesity. J Biol Chem 278: 1719017197, 2003.

25. Baines CP, Kaiser RA, Purcell NH, Blair NS, Osinska H, Hambleton MA, Brunskill EW, Sayen MR, Gottlieb RA, Dorn GW, Robbins J, and Molkentin JD. Loss of cyclophilin $\mathrm{D}$ reveals a critical role for mitochondrial permeability transition in cell death. Nature 434: 658-662, 2005.

26. Baines CP, Kaiser RA, Sheiko T, Craigen WJ, and Molkentin JD. Voltage-dependent anion channels are dispensable for mitochondrial-dependent cell death. Nat Cell Biol 9: 550-555, 2007.

27. Barber SC, Mead RJ, and Shaw PJ. Oxidative stress in ALS: a mechanism of neurodegeneration and a therapeutic target. Biochim Biophys Acta 1762: 1051-1067, 2006.

28. Barsoum MJ, Yuan H, Gerencser AA, Liot G, Kushnareva Y, Graber S, Kovacs I, Lee WD, Waggoner J, Cui J, White AD, Bossy B, Martinou JC, Youle RJ, Lipton SA, Ellisman $\mathrm{MH}$, Perkins GA, and Bossy-Wetzel E. Nitric oxide-induced mitochondrial fission is regulated by dynamin-related GTPases in neurons. EMBO J 25: 3900-3911, 2006. 
29. Bartel J, Bartz T, Wolf C, Charkiewicz E, Kuhbacher M, Pohl T, and Kyriakopoulos A. Activity of the glutathione peroxidase-2. Differences in the selenium-dependent expression between colon and small intestine. Cancer Genomics Proteomics 4: 369-372, 2007.

30. Basova LV, Kurnikov IV, Wang L, Ritov VB, Belikova NA, Vlasova, II, Pacheco AA, Winnica DE, Peterson J, Bayir H, Waldeck DH, and Kagan VE. Cardiolipin switch in mitochondria: shutting off the reduction of cytochrome $\mathrm{c}$ and turning on the peroxidase activity. Biochemistry 46: 34233434, 2007.

31. Basso E, Fante L, Fowlkes J, Petronilli V, Forte MA, and Bernardi P. Properties of the permeability transition pore in mitochondria devoid of Cyclophilin D. J Biol Chem 280: 18558-18561, 2005.

32. Baty JW, Hampton MB, and Winterbourn CC. Proteomic detection of hydrogen peroxide-sensitive thiol proteins in Jurkat cells. Biochem J 389: 785-795, 2005.

33. Baur JA, Pearson KJ, Price NL, Jamieson HA, Lerin C, Kalra A, Prabhu VV, Allard JS, Lopez-Lluch G, Lewis K, Pistell PJ, Poosala S, Becker KG, Boss O, Gwinn D, Wang M, Ramaswamy S, Fishbein KW, Spencer RG, Lakatta EG, Le Couteur D, Shaw RJ, Navas P, Puigserver P, Ingram DK, de Cabo R, and Sinclair DA. Resveratrol improves health and survival of mice on a high-calorie diet. Nature 444: 337-342, 2006.

34. Bayir H, Fadeel B, Palladino MJ, Witasp E, Kurnikov IV, Tyurina YY, Tyurin VA, Amoscato AA, Jiang J, Kochanek PM, DeKosky ST, Greenberger JS, Shvedova AA, and Kagan VE. Apoptotic interactions of cytochrome c: redox flirting with anionic phospholipids within and outside of mitochondria. Biochim Biophys Acta 1757: 648-659, 2006.

35. Bayley JP and Devilee P. Warburg tumours and the mechanisms of mitochondrial tumour suppressor genes. Barking up the right tree? Curr Opin Genet Dev 20: 324-329, 2010.

36. Beer SM, Taylor ER, Brown SE, Dahm CC, Costa NJ, Runswick MJ, and Murphy MP. Glutaredoxin 2 catalyzes the reversible oxidation and glutathionylation of mitochondrial membrane thiol proteins: implications for mitochondrial redox regulation and antioxidant defense. J Biol Chem 279: 47939-47951, 2004.

37. Benton CR, Holloway GP, Han XX, Yoshida Y, Snook LA, Lally J, Glatz JF, Luiken JJ, Chabowski A, and Bonen A. Increased levels of peroxisome proliferator-activated receptor gamma, coactivator 1 alpha (PGC-1alpha) improve lipid utilisation, insulin signalling and glucose transport in skeletal muscle of lean and insulin-resistant obese Zucker rats. Diabetologia 53: 2008-2019, 2010.

38. Berry CE and Hare JM. Xanthine oxidoreductase and cardiovascular disease: molecular mechanisms and pathophysiological implications. J Physiol 555: 589-606, 2004.

39. Bhusari SS, Dobosy JR, Fu V, Almassi N, Oberley T, and Jarrard DF. Superoxide dismutase 1 knockdown induces oxidative stress and DNA methylation loss in the prostate. Epigenetics 5: 402-409, 2010.

40. Bien M, Longen S, Wagener N, Chwalla I, Herrmann JM, and Riemer J. Mitochondrial disulfide bond formation is driven by intersubunit electron transfer in Erv1 and proofread by glutathione. Mol Cell 37: 516-528, 2010.

41. Bienengraeber M, Ozcan C, and Terzic A. Stable transfection of UCP1 confers resistance to hypoxia/reoxygenation in a heart-derived cell line. J Mol Cell Cardiol 35: 861-865, 2003.

42. Blankenberg S, Rupprecht HJ, Bickel C, Torzewski M, Hafner G, Tiret L, Smieja M, Cambien F, Meyer J, and
Lackner KJ. Glutathione peroxidase 1 activity and cardiovascular events in patients with coronary artery disease. $N$ Engl J Med 349: 1605-1613, 2003.

43. Bodyak N, Rigor DL, Chen YS, Han Y, Bisping E, Pu WT, and Kang PM. Uncoupling protein 2 modulates cell viability in adult rat cardiomyocytes. Am J Physiol Heart Circ Physiol 293: H829-H835, 2007.

44. Borra MT, Smith BC, and Denu JM. Mechanism of human SIRT1 activation by resveratrol. J Biol Chem 280: 1718717195, 2005.

45. Bossy B, Petrilli A, Klinglmayr E, Chen J, Lutz-Meindl U, Knott AB, Masliah E, Schwarzenbacher R, and BossyWetzel E. S-Nitrosylation of DRP1 does not affect enzymatic activity and is not specific to Alzheimer's disease. $J$ Alzheimers Dis 20 Suppl 2: S513-S526, 2010.

46. Bossy-Wetzel E, Newmeyer DD, and Green DR. Mitochondrial cytochrome $\mathrm{c}$ release in apoptosis occurs upstream of DEVD-specific caspase activation and independently of mitochondrial transmembrane depolarization. EMBO J 17: 37-49, 1998.

47. Boutilier RG. Mechanisms of cell survival in hypoxia and hypothermia. J Exp Biol 204: 3171-3181, 2001.

48. Brady NR, Elmore SP, van Beek JJ, Krab K, Courtoy PJ, Hue $\mathrm{L}$, and Westerhoff HV. Coordinated behavior of mitochondria in both space and time: a reactive oxygen speciesactivated wave of mitochondrial depolarization. Biophys $J$ 87: 2022-2034, 2004.

49. Brand MD and Esteves TC. Physiological functions of the mitochondrial uncoupling proteins UCP2 and UCP3. Cell Metab 2: 85-93, 2005.

50. Brennan JP, Bardswell SC, Burgoyne JR, Fuller W, Schroder E, Wait R, Begum S, Kentish JC, and Eaton P. Oxidantinduced activation of type I protein kinase $\mathrm{A}$ is mediated by RI subunit interprotein disulfide bond formation. J Biol Chem 281: 21827-21836, 2006.

51. Brennan JP, Wait R, Begum S, Bell JR, Dunn MJ, and Eaton $P$. Detection and mapping of widespread intermolecular protein disulfide formation during cardiac oxidative stress using proteomics with diagonal electrophoresis. J Biol Chem 279: 41352-41360, 2004.

52. Brookes PS. Mitochondrial $\mathrm{H}(+)$ leak and ROS generation: an odd couple. Free Radic Biol Med 38: 12-23, 2005.

53. Brunelle JK, Bell EL, Quesada NM, Vercauteren K, Tiranti V, Zeviani M, Scarpulla RC, and Chandel NS. Oxygen sensing requires mitochondrial ROS but not oxidative phosphorylation. Cell Metab 1: 409-414, 2005.

54. Budihardjo I, Oliver H, Lutter M, Luo X, and Wang X. Biochemical pathways of caspase activation during apoptosis. Annu Rev Cell Dev Biol 15: 269-290, 1999.

55. Budzinska M, Galganska H, Karachitos A, Wojtkowska M, and $\mathrm{Kmita} \mathrm{H}$. The TOM complex is involved in the release of superoxide anion from mitochondria. J Bioenerg Biomembr 41: 361-367, 2009.

56. Burnett C, Valentini S, Cabreiro F, Goss M, Somogyvari M, Piper MD, Hoddinott M, Sutphin GL, Leko V, McElwee JJ, Vazquez-Manrique RP, Orfila AM, Ackerman D, Au C, Vinti G, Riesen M, Howard K, Neri C, Bedalov A, Kaeberlein M, Soti C, Partridge L, and Gems D. Absence of effects of Sir2 overexpression on lifespan in C. elegans and Drosophila. Nature 477: 482485, 2011.

57. Burwell LS, Nadtochiy SM, Tompkins AJ, Young S, and Brookes PS. Direct evidence for S-nitrosation of mitochondrial complex I. Biochem J 394: 627-634, 2006. 
58. Busse R and Mulsch A. Calcium-dependent nitric oxide synthesis in endothelial cytosol is mediated by calmodulin. FEBS Lett 265: 133-136, 1990.

59. Butzer U, Weidenbach H, Gansauge S, Gansauge F, Beger HG, and Nussler AK. Increased oxidative stress in the RAW 264.7 macrophage cell line is partially mediated via the S-nitrosothiol-induced inhibition of glutathione reductase. FEBS Lett 445: 274-278, 1999.

60. Cam H, Balciunaite E, Blais A, Spektor A, Scarpulla RC, Young R, Kluger Y, and Dynlacht BD. A common set of gene regulatory networks links metabolism and growth inhibition. Mol Cell 16: 399-411, 2004.

61. Camici GG, Cosentino F, Tanner FC, and Luscher TF. The role of p66Shc deletion in age-associated arterial dysfunction and disease states. J Appl Physiol 105: 1628-1631, 2008.

62. Canto C, Gerhart-Hines Z, Feige JN, Lagouge M, Noriega L, Milne JC, Elliott PJ, Puigserver P, and Auwerx J. AMPK regulates energy expenditure by modulating NAD+ metabolism and SIRT1 activity. Nature 458: 1056-1060, 2009.

63. Cappadoro M, Giribaldi G, O’Brien E, Turrini F, Mannu F, Ulliers D, Simula G, Luzzatto L, and Arese P. Early phagocytosis of glucose-6-phosphate dehydrogenase (G6PD)-deficient erythrocytes parasitized by Plasmodium falciparum may explain malaria protection in G6PD deficiency. Blood 92: 2527-2534, 1998.

64. Castilho RF, Kowaltowski AJ, Meinicke AR, Bechara EJ, and Vercesi AE. Permeabilization of the inner mitochondrial membrane by $\mathrm{Ca}^{2+}$ ions is stimulated by t-butyl hydroperoxide and mediated by reactive oxygen species generated by mitochondria. Free Radic Biol Med 18: 479-486, 1995.

65. Chae HZ, Kim HJ, Kang SW, and Rhee SG. Characterization of three isoforms of mammalian peroxiredoxin that reduce peroxides in the presence of thioredoxin. Diabetes Res Clin Pract 45: 101-112, 1999.

66. Chae HZ, Robison K, Poole LB, Church G, Storz G, and Rhee SG. Cloning and sequencing of thiol-specific antioxidant from mammalian brain: alkyl hydroperoxide reductase and thiol-specific antioxidant define a large family of antioxidant enzymes. Proc Natl Acad Sci U S A 91: 70177021, 1994.

67. Chambers JW and Lograsso PV. Mitochondrial c-Jun Nterminal kinase (JNK) signaling initiates physiological changes resulting in amplification of reactive oxygen species generation. J Biol Chem 286: 16052-16062, 2010.

68. Chan CB and Harper ME. Uncoupling proteins: role in insulin resistance and insulin insufficiency. Curr Diabetes Rev 2: 271-283, 2006.

69. Chan SY, Zhang YY, Hemann C, Mahoney CE, Zweier JL, and Loscalzo J. MicroRNA-210 controls mitochondrial metabolism during hypoxia by repressing the iron-sulfur cluster assembly proteins ISCU1/2. Cell Metab 10: 273-284, 2009.

70. Chance B and Hollunger G. The interaction of energy and electron transfer reactions in mitochondria. I. General properties and nature of the products of succinate-linked reduction of pyridine nucleotide. J Biol Chem 236: 1534$1543,1961$.

71. Chance B, Sies H, and Boveris A. Hydroperoxide metabolism in mammalian organs. Physiol Rev 59: 527-605, 1979.

72. Chandel NS and Budinger GR. The cellular basis for diverse responses to oxygen. Free Radic Biol Med 42: 165-174, 2007.
73. Chang TS, Cho CS, Park S, Yu S, Kang SW, and Rhee SG. Peroxiredoxin III, a mitochondrion-specific peroxidase, regulates apoptotic signaling by mitochondria. J Biol Chem 279: 41975-41984, 2004.

74. Chang TS, Jeong W, Woo HA, Lee SM, Park S, and Rhee SG. Characterization of mammalian sulfiredoxin and its reactivation of hyperoxidized peroxiredoxin through reduction of cysteine sulfinic acid in the active site to cysteine. J Biol Chem 279: 50994-51001, 2004.

75. Chattopadhyay M and Valentine JS. Aggregation of copper-zinc superoxide dismutase in familial and sporadic ALS. Antioxid Redox Signal 11: 1603-1614, 2009.

76. Chen J, Chen CL, Rawale S, Chen CA, Zweier JL, Kaumaya PT, and Chen YR. Peptide-based antibodies against glutathione-binding domains suppress superoxide production mediated by mitochondrial complex I. J Biol Chem 285: 3168-3180, 2010.

77. Chen K, Kirber MT, Xiao H, Yang Y, and Keaney JF, Jr. Regulation of ROS signal transduction by NADPH oxidase 4 localization. J Cell Biol 181: 1129-1139, 2008.

78. Chen K, Thomas SR, Albano A, Murphy MP, and Keaney JF, Jr. Mitochondrial function is required for hydrogen peroxide-induced growth factor receptor transactivation and downstream signaling. J Biol Chem 279: 35079-35086, 2004.

79. Chen Y, Cai J, Murphy TJ, and Jones DP. Overexpressed human mitochondrial thioredoxin confers resistance to oxidant-induced apoptosis in human osteosarcoma cells. J Biol Chem 277: 33242-33248, 2002.

80. Chen YR, Wang X, Templeton D, Davis RJ, and Tan TH. The role of c-Jun N-terminal kinase (JNK) in apoptosis induced by ultraviolet $\mathrm{C}$ and gamma radiation. Duration of JNK activation may determine cell death and proliferation. J Biol Chem 271: 31929-31936, 1996.

81. Chen Z, Li Y, Zhang H, Huang P, and Luthra R. Hypoxiaregulated microRNA-210 modulates mitochondrial function and decreases ISCU and COX10 expression. Oncogene 29: 4362-4368, 2010.

82. Cheng YY and Qian PC. The effect of selenium-fortified table salt in the prevention of Keshan disease on a population of 1.05 million. Biomed Environ Sci 3: 422-428, 1990.

83. Cheng $Z$, Tseng $Y$, and White MF. Insulin signaling meets mitochondria in metabolism. Trends Endocrinol Metab 21: 589-598, 2010.

84. Chin L, Artandi SE, Shen Q, Tam A, Lee SL, Gottlieb GJ, Greider CW, and DePinho RA. p53 deficiency rescues the adverse effects of telomere loss and cooperates with telomere dysfunction to accelerate carcinogenesis. Cell 97: 527$538,1999$.

85. Cho CS, Lee S, Lee GT, Woo HA, Choi EJ, and Rhee SG. Irreversible inactivation of glutathione peroxidase 1 and reversible inactivation of peroxiredoxin II by $\mathrm{H}_{2} \mathrm{O}_{2}$ in red blood cells. Antioxid Redox Signal 12: 1235-1246, 2010.

86. Cho DH, Nakamura T, Fang J, Cieplak P, Godzik A, Gu Z, and Lipton SA. S-nitrosylation of Drp1 mediates betaamyloid-related mitochondrial fission and neuronal injury. Science 324: 102-105, 2009.

87. Choi CS, Befroy DE, Codella R, Kim S, Reznick RM, Hwang YJ, Liu ZX, Lee HY, Distefano A, Samuel VT, Zhang D, Cline GW, Handschin C, Lin J, Petersen KF, Spiegelman BM, and Shulman GI. Paradoxical effects of increased expression of PGC-1alpha on muscle mitochondrial function and insulin-stimulated muscle glucose metabolism. Proc Natl Acad Sci U S A 105: 19926-19931, 2008. 
88. Choi MH, Lee IK, Kim GW, Kim BU, Han YH, Yu DY, Park HS, Kim KY, Lee JS, Choi C, Bae YS, Lee BI, Rhee SG, and Kang SW. Regulation of PDGF signalling and vascular remodelling by peroxiredoxin II. Nature 435: 347-353, 2005.

89. Choi YB and Lipton SA. Redox modulation of the NMDA receptor. Cell Mol Life Sci 57: 1535-1541, 2000.

90. Choi YJ, Cho SK, Hwang KC, Park C, Kim JH, Park SB, Hwang S, and Kim JH. Nm23-M5 mediates round and elongated spermatid survival by regulating GPX-5 levels. FEBS Lett 583: 1292-1298, 2009.

91. Chouchani ET, Hurd TR, Nadtochiy SM, Brookes PS, Fearnley IM, Lilley KS, Smith RA, and Murphy MP. Identification of S-nitrosated mitochondrial proteins by $\mathrm{S}$ nitrosothiol difference in gel electrophoresis (SNO-DIGE): implications for the regulation of mitochondrial function by reversible S-nitrosation. Biochem J 430: 49-59, 2010.

92. Christou H, Morita T, Hsieh CM, Koike H, Arkonac B, Perrella MA, and Kourembanas S. Prevention of hypoxiainduced pulmonary hypertension by enhancement of endogenous heme oxygenase-1 in the rat. Circ Res 86: 1224 1229, 2000.

93. Cipolat S, Martins de Brito O, Dal Zilio B, and Scorrano L. OPA1 requires mitofusin 1 to promote mitochondrial fusion. Proc Natl Acad Sci U S A 101: 15927-15932, 2004.

94. Civitarese $\mathrm{AE}$ and Ravussin E. Mitochondrial energetics and insulin resistance. Endocrinology 149: 950-954, 2008.

95. Clavreul N, Bachschmid MM, Hou X, Shi C, Idrizovic A, Ido Y, Pimentel D, and Cohen RA. S-glutathiolation of p21ras by peroxynitrite mediates endothelial insulin resistance caused by oxidized low-density lipoprotein. Arterioscler Thromb Vasc Biol 26: 2454-2461, 2006.

96. Claypool SM, Oktay Y, Boontheung P, Loo JA, and Koehler $\mathrm{CM}$. Cardiolipin defines the interactome of the major ADP/ ATP carrier protein of the mitochondrial inner membrane. J Cell Biol 182: 937-950, 2008.

97. Cleeter MW, Cooper JM, Darley-Usmar VM, Moncada S, and Schapira AH. Reversible inhibition of cytochrome c oxidase, the terminal enzyme of the mitochondrial respiratory chain, by nitric oxide. Implications for neurodegenerative diseases. FEBS Lett 345: 50-54, 1994.

98. Clementi E, Brown GC, Feelisch M, and Moncada S. Persistent inhibition of cell respiration by nitric oxide: crucial role of S-nitrosylation of mitochondrial complex I and protective action of glutathione. Proc Natl Acad Sci U S A 95: 7631-7636, 1998

99. Connor KM, Subbaram S, Regan KJ, Nelson KK, Mazurkiewicz JE, Bartholomew PJ, Aplin AE, Tai YT, AguirreGhiso J, Flores SC, and Melendez JA. Mitochondrial $\mathrm{H}_{2} \mathrm{O}_{2}$ regulates the angiogenic phenotype via PTEN oxidation. $J$ Biol Chem 280: 16916-16924, 2005.

100. Conrad M, Jakupoglu C, Moreno SG, Lippl S, Banjac A, Schneider M, Beck H, Hatzopoulos AK, Just U, Sinowatz F, Schmahl W, Chien KR, Wurst W, Bornkamm GW, and Brielmeier M. Essential role for mitochondrial thioredoxin reductase in hematopoiesis, heart development, and heart function. Mol Cell Biol 24: 9414-9423, 2004.

101. Costantini P, Belzacq AS, Vieira HL, Larochette N, de Pablo MA, Zamzami N, Susin SA, Brenner C, and Kroemer G. Oxidation of a critical thiol residue of the adenine nucleotide translocator enforces Bcl-2-independent permeability transition pore opening and apoptosis. Oncogene 19: 307314, 2000.

102. Costantini P, Chernyak BV, Petronilli V, and Bernardi P. Selective inhibition of the mitochondrial permeability transition pore at the oxidation-reduction sensitive dithiol by monobromobimane. FEBS Lett 362: 239-242, 1995.

103. Crack PJ, Taylor JM, Ali U, Mansell A, and Hertzog PJ. Potential contribution of NF-kappaB in neuronal cell death in the glutathione peroxidase- 1 knockout mouse in response to ischemia-reperfusion injury. Stroke 37: 1533-1538, 2006.

104. Cumming RC, Andon NL, Haynes PA, Park M, Fischer $\mathrm{WH}$, and Schubert D. Protein disulfide bond formation in the cytoplasm during oxidative stress. J Biol Chem 279: 21749-21758, 2004.

105. Dabir DV, Leverich EP, Kim SK, Tsai FD, Hirasawa M, Knaff DB, and Koehler CM. A role for cytochrome $\mathrm{c}$ and cytochrome c peroxidase in electron shuttling from Erv1. EMBO J 26: 4801-4811, 2007.

106. Dabkowski ER, Williamson CL, and Hollander JM. Mitochondria-specific transgenic overexpression of phospholipid hydroperoxide glutathione peroxidase (GPx4) attenuates ischemia/reperfusion-associated cardiac dysfunction. Free Radic Biol Med 45: 855-865, 2008.

107. Dagda RK, Cherra SJ, 3rd, Kulich SM, Tandon A, Park D, and Chu CT. Loss of PINK1 function promotes mitophagy through effects on oxidative stress and mitochondrial fission. J Biol Chem 284: 13843-13855, 2009.

108. Dahm CC, Moore K, and Murphy MP. Persistent Snitrosation of complex I and other mitochondrial membrane proteins by S-nitrosothiols but not nitric oxide or peroxynitrite: implications for the interaction of nitric oxide with mitochondria. J Biol Chem 281: 10056-10065, 2006.

109. Davidson SM and Duchen MR. Endothelial mitochondria: contributing to vascular function and disease. Circ Res 100: 1128-1141, 2007.

110. de Bilbao F, Arsenijevic D, Vallet P, Hjelle OP, Ottersen OP, Bouras C, Raffin Y, Abou K, Langhans W, Collins S, Plamondon J, Alves-Guerra MC, Haguenauer A, Garcia I, Richard D, Ricquier D, and Giannakopoulos P. Resistance to cerebral ischemic injury in UCP2 knockout mice: evidence for a role of UCP2 as a regulator of mitochondrial glutathione levels. J Neurochem 89: 1283-1292, 2004.

111. de Cavanagh EM, Piotrkowski B, Basso N, Stella I, Inserra F, Ferder L, and Fraga CG. Enalapril and losartan attenuate mitochondrial dysfunction in aged rats. FASEB J 17: 10961098, 2003.

112. de Cavanagh EM, Toblli JE, Ferder L, Piotrkowski B, Stella I, and Inserra F. Renal mitochondrial dysfunction in spontaneously hypertensive rats is attenuated by losartan but not by amlodipine. Am J Physiol Regul Integr Comp Physiol 290: R1616-R1625, 2006.

113. De Palma C, Falcone S, Pisoni S, Cipolat S, Panzeri C, Pambianco S, Pisconti A, Allevi R, Bassi MT, Cossu G, Pozzan T, Moncada S, Scorrano L, Brunelli S, and Clementi E. Nitric oxide inhibition of Drp1-mediated mitochondrial fission is critical for myogenic differentiation. Cell Death Differ 17: 1684-1696, 2010.

114. Denu JM and Tanner KG. Specific and reversible inactivation of protein tyrosine phosphatases by hydrogen peroxide: evidence for a sulfenic acid intermediate and implications for redox regulation. Biochemistry 37: 56335642, 1998.

115. Desmard M, Boczkowski J, Poderoso J, and Motterlini R. Mitochondrial and cellular heme-dependent proteins as targets for the bioactive function of the heme oxygenase/ carbon monoxide system. Antioxid Redox Signal 9: 2139$2155,2007$. 
116. Di Fonzo A, Ronchi D, Lodi T, Fassone E, Tigano M, Lamperti C, Corti S, Bordoni A, Fortunato F, Nizzardo M, Napoli L, Donadoni C, Salani S, Saladino F, Moggio M, Bresolin N, Ferrero I, and Comi GP. The mitochondrial disulfide relay system protein GFER is mutated in autosomal-recessive myopathy with cataract and combined respiratory-chain deficiency. Am J Hum Genet 84: 594-604, 2009.

117. Dienel GA and Hertz L. Glucose and lactate metabolism during brain activation. J Neurosci Res 66: 824-838, 2001.

118. Dikalov SI, Dikalova AE, Bikineyeva AT, Schmidt HH, Harrison DG, and Griendling KK. Distinct roles of Nox1 and Nox4 in basal and angiotensin II-stimulated superoxide and hydrogen peroxide production. Free Radic Biol Med 45: 1340-1351, 2008.

119. Diotte NM, Xiong Y, Gao J, Chua BH, and Ho YS. Attenuation of doxorubicin-induced cardiac injury by mitochondrial glutaredoxin 2. Biochim Biophys Acta 1793: 427-438, 2009.

120. Diwakar L, Kenchappa RS, Annepu J, and Ravindranath V. Downregulation of glutaredoxin but not glutathione loss leads to mitochondrial dysfunction in female mice CNS: implications in excitotoxicity. Neurochem Int 51: 37-46, 2007.

121. Doughan AK, Harrison DG, and Dikalov SI. Molecular mechanisms of angiotensin II-mediated mitochondrial dysfunction: linking mitochondrial oxidative damage and vascular endothelial dysfunction. Circ Res 102: 488-496, 2008.

122. Dromparis P, Sutendra G, and Michelakis ED. The role of mitochondria in pulmonary vascular remodeling. $\mathrm{J} \mathrm{Mol}$ Med 88: 1003-1010, 2010.

123. Elrod JW, Wong R, Mishra S, Vagnozzi RJ, Sakthievel B, Goonasekera SA, Karch J, Gabel S, Farber J, Force T, Brown JH, Murphy E, and Molkentin JD. Cyclophilin D controls mitochondrial pore-dependent $\mathrm{Ca}(2+)$ exchange, metabolic flexibility, and propensity for heart failure in mice. J Clin Invest 120: 3680-3687, 2010.

124. Enoksson M, Fernandes AP, Prast S, Lillig $\mathrm{CH}$, Holmgren A, and Orrenius S. Overexpression of glutaredoxin 2 attenuates apoptosis by preventing cytochrome c release. Biochem Biophys Res Commun 327: 774-779, 2005.

125. Eskes R, Antonsson B, Osen-Sand A, Montessuit S, Richter C, Sadoul R, Mazzei G, Nichols A, and Martinou JC. Bax-induced cytochrome $C$ release from mitochondria is independent of the permeability transition pore but highly dependent on Mg2 + ions. J Cell Biol 143: 217-224, 1998.

126. Esposito LA, Kokoszka JE, Waymire KG, Cottrell B, MacGregor GR, and Wallace DC. Mitochondrial oxidative stress in mice lacking the glutathione peroxidase-1 gene. Free Radic Biol Med 28: 754-766, 2000.

127. Eto M, Kajihara N, Morita S, and Tominaga R. A novel electron paramagnetic resonance spin-probe technique demonstrates the relation between the production of hydroxyl radicals and ischemia-reperfusion injury. Eur $J$ Cardiothorac Surg 39: 465-470, 2011.

128. Fadini GP, Albiero M, Menegazzo L, Boscaro E, Pagnin E, Iori E, Cosma C, Lapolla A, Pengo V, Stendardo M, Agostini C, Pelicci PG, Giorgio M, and Avogaro A. The redox enzyme p66Shc contributes to diabetes and ischemiainduced delay in cutaneous wound healing. Diabetes 59: 2306-2314, 2010.

129. Favaro E, Ramachandran A, McCormick R, Gee H, Blancher C, Crosby M, Devlin C, Blick C, Buffa F, Li JL, Vojnovic B, Pires das Neves R, Glazer P, Iborra F, Ivan M,
Ragoussis J, and Harris AL. MicroRNA-210 regulates mitochondrial free radical response to hypoxia and krebs cycle in cancer cells by targeting iron sulfur cluster protein ISCU. PLoS One 5: e10345, 2010.

130. Ferri A, Fiorenzo P, Nencini M, Cozzolino M, Pesaresi MG, Valle C, Sepe S, Moreno S, and Carri MT. Glutaredoxin 2 prevents aggregation of mutant SOD1 in mitochondria and abolishes its toxicity. Hum Mol Genet 19: 4529-4542, 2010.

131. Fijalkowska I, Xu W, Comhair SA, Janocha AJ, Mavrakis LA, Krishnamachary B, Zhen L, Mao T, Richter A, Erzurum SC, and Tuder RM. Hypoxia inducible-factorlalpha regulates the metabolic shift of pulmonary hypertensive endothelial cells. Am J Pathol 176: 1130-1138, 2010.

132. Fisher AB. Peroxiredoxin 6: a bifunctional enzyme with glutathione peroxidase and phospholipase A2 activities. Antioxid Redox Signal 15: 831-844, 2011.

133. Flohe L, Loschen G, Gunzler WA, and Eichele E. Glutathione peroxidase, V. The kinetic mechanism. Hoppe Seylers Z Physiol Chem 353: 987-999, 1972.

134. Forgione MA, Cap A, Liao R, Moldovan NI, Eberhardt RT, Lim CC, Jones J, Goldschmidt-Clermont PJ, and Loscalzo J. Heterozygous cellular glutathione peroxidase deficiency in the mouse: abnormalities in vascular and cardiac function and structure. Circulation 106: 1154-1158, 2002.

135. Forgione MA, Weiss N, Heydrick S, Cap A, Klings ES, Bierl C, Eberhardt RT, Farber HW, and Loscalzo J. Cellular glutathione peroxidase deficiency and endothelial dysfunction. Am J Physiol Heart Circ Physiol 282: H1255-H1261, 2002.

136. Forman HJ, Maiorino M, and Ursini F. Signaling functions of reactive oxygen species. Biochemistry 49: 835-842, 2010.

137. Fourquet S, Huang ME, D'Autreaux B, and Toledano MB. The dual functions of thiol-based peroxidases in $\mathrm{H}_{2} \mathrm{O}_{2}$ scavenging and signaling. Antioxid Redox Signal 10: 15651576, 2008.

138. Frazier AE, Kiu C, Stojanovski D, Hoogenraad NJ, and Ryan MT. Mitochondrial morphology and distribution in mammalian cells. Biol Chem 387: 1551-1558, 2006.

139. Freedman JE, Loscalzo J, Benoit SE, Valeri CR, Barnard MR, and Michelson AD. Decreased platelet inhibition by nitric oxide in two brothers with a history of arterial thrombosis. J Clin Invest 97: 979-987, 1996.

140. Fu Y, Sies H, and Lei XG. Opposite roles of seleniumdependent glutathione peroxidase-1 in superoxide generator diquat- and peroxynitrite-induced apoptosis and signaling. J Biol Chem 276: 43004-43009, 2001.

141. Fujino G, Noguchi T, Matsuzawa A, Yamauchi S, Saitoh M, Takeda K, and Ichijo H. Thioredoxin and TRAF family proteins regulate reactive oxygen species-dependent activation of ASK1 through reciprocal modulation of the Nterminal homophilic interaction of ASK1. Mol Cell Biol 27: 8152-8163, 2007.

142. Fujisawa K, Nishikawa T, Kukidome D, Imoto K, Yamashiro T, Motoshima H, Matsumura T, and Araki E. TZDs reduce mitochondrial ROS production and enhance mitochondrial biogenesis. Biochem Biophys Res Commun 379: 4348, 2009.

143. Fukuda R, Zhang H, Kim JW, Shimoda L, Dang CV, and Semenza GL. HIF-1 regulates cytochrome oxidase subunits to optimize efficiency of respiration in hypoxic cells. Cell 129: 111-122, 2007.

144. Fukui M, Choi HJ, and Zhu BT. Mechanism for the protective effect of resveratrol against oxidative stress-induced neuronal death. Free Radic Biol Med 49: 800-813, 2010. 
145. Fuyu Y. Keshan disease and mitochondrial cardiomyopathy. Sci China C Life Sci 49: 513-518, 2006.

146. Gaetani GF, Galiano S, Canepa L, Ferraris AM, and Kirkman HN. Catalase and glutathione peroxidase are equally active in detoxification of hydrogen peroxide in human erythrocytes. Blood 73: 334-339, 1989.

147. Garlid KD, Orosz DE, Modriansky M, Vassanelli S, and Jezek P. On the mechanism of fatty acid-induced proton transport by mitochondrial uncoupling protein. J Biol Chem 271: 2615-2620, 1996.

148. Geisler S, Holmstrom KM, Treis A, Skujat D, Weber SS, Fiesel FC, Kahle PJ, and Springer W. The PINK1/Parkinmediated mitophagy is compromised by PD-associated mutations. Autophagy 6: 871-878, 2010.

149. Genova ML, Ventura B, Giuliano G, Bovina C, Formiggini G, Parenti Castelli G, and Lenaz G. The site of production of superoxide radical in mitochondrial Complex I is not a bound ubisemiquinone but presumably iron-sulfur cluster N2. FEBS Lett 505: 364-368, 2001.

150. Gerhart-Hines Z, Rodgers JT, Bare O, Lerin C, Kim SH, Mostoslavsky R, Alt FW, Wu Z, and Puigserver P. Metabolic control of muscle mitochondrial function and fatty acid oxidation through SIRT1/PGC-1alpha. EMBO J 26: 1913-1923, 2007.

151. Gertz M, Fischer F, Wolters D, and Steegborn C. Activation of the lifespan regulator p66Shc through reversible disulfide bond formation. Proc Natl Acad Sci U S A 105: 57055709, 2008.

152. Ghafourifar P and Richter C. Nitric oxide synthase activity in mitochondria. FEBS Lett 418: 291-296, 1997.

153. Giorgio M, Migliaccio E, Orsini F, Paolucci D, Moroni M, Contursi C, Pelliccia G, Luzi L, Minucci S, Marcaccio M, Pinton P, Rizzuto R, Bernardi P, Paolucci F, and Pelicci PG. Electron transfer between cytochrome $\mathrm{c}$ and p66Shc generates reactive oxygen species that trigger mitochondrial apoptosis. Cell 122: 221-233, 2005.

154. Gleyzer N, Vercauteren K, and Scarpulla RC. Control of mitochondrial transcription specificity factors (TFB1M and TFB2M) by nuclear respiratory factors (NRF-1 and NRF-2) and PGC-1 family coactivators. Mol Cell Biol 25: 1354-1366, 2005.

155. Go YM and Jones DP. Cysteine/cystine redox signaling in cardiovascular disease. Free Radic Biol Med 50: 495-509, 2011.

156. Go YM and Jones DP. Redox compartmentalization in eukaryotic cells. Biochim Biophys Acta 1780: 1273-1290, 2008.

157. Go YM, Park H, Koval M, Orr M, Reed M, Liang Y, Smith D, Pohl J, and Jones DP. A key role for mitochondria in endothelial signaling by plasma cysteine/cystine redox potential. Free Radic Biol Med 48: 275-283, 2010.

158. Gongora MC, Qin Z, Laude K, Kim HW, McCann L, Folz JR, Dikalov S, Fukai T, and Harrison DG. Role of extracellular superoxide dismutase in hypertension. Hypertension 48: 473-481, 2006.

159. Griffiths EJ. Mitochondria-potential role in cell life and death. Cardiovasc Res 46: 24-27, 2000.

160. Gulshan K, Rovinsky SA, Coleman ST, and Moye-Rowley WS. Oxidant-specific folding of Yap1p regulates both transcriptional activation and nuclear localization. J Biol Chem 280: 40524-40533, 2005.

161. Gunter TE and Pfeiffer DR. Mechanisms by which mitochondria transport calcium. Am J Physiol 258: C755-C786, 1990.

162. Guo YL, Baysal K, Kang B, Yang LJ, and Williamson JR. Correlation between sustained c-Jun $\mathrm{N}$-terminal protein kinase activation and apoptosis induced by tumor necrosis factor-alpha in rat mesangial cells. J Biol Chem 273: 40274034, 1998.

163. Gutscher M, Pauleau AL, Marty L, Brach T, Wabnitz GH, Samstag Y, Meyer AJ, and Dick TP. Real-time imaging of the intracellular glutathione redox potential. Nat Methods 5: 553-559, 2008.

164. Guzy RD, Hoyos B, Robin E, Chen H, Liu L, Mansfield KD, Simon MC, Hammerling U, and Schumacker PT. Mitochondrial complex III is required for hypoxia-induced ROS production and cellular oxygen sensing. Cell Metab 1: 401-408, 2005.

165. Haigis MC, Mostoslavsky R, Haigis KM, Fahie K, Christodoulou DC, Murphy AJ, Valenzuela DM, Yancopoulos GD, Karow M, Blander G, Wolberger C, Prolla TA, Weindruch R, Alt FW, and Guarente L. SIRT4 inhibits glutamate dehydrogenase and opposes the effects of calorie restriction in pancreatic beta cells. Cell 126: 941-954, 2006.

166. Hajdu J, Wyss SR, and Aebi H. Properties of human erythrocyte catalases after crosslinking with bifunctional reagents. Symmetry of the quaternary structure. Eur J Biochem 80: 199-207, 1977.

167. Halestrap AP, Woodfield KY, and Connern CP. Oxidative stress, thiol reagents, and membrane potential modulate the mitochondrial permeability transition by affecting nucleotide binding to the adenine nucleotide translocase. J Biol Chem 272: 3346-3354, 1997.

168. Han D, Antunes F, Canali R, Rettori D, and Cadenas E. Voltage-dependent anion channels control the release of the superoxide anion from mitochondria to cytosol. J Biol Chem 278: 5557-5563, 2003.

169. Handy DE, Lubos E, Yang Y, Galbraith JD, Kelly N, Zhang YY, Leopold JA, and Loscalzo J. Glutathione peroxidase-1 regulates mitochondrial function to modulate redoxdependent cellular responses. J Biol Chem 284: 11913-11921, 2009.

170. Hanschmann EM, Lonn ME, Schutte LD, Funke M, Godoy JR, Eitner S, Hudemann C, and Lillig CH. Both thioredoxin 2 and glutaredoxin 2 contribute to the reduction of the mitochondrial 2-Cys peroxiredoxin Prx3. J Biol Chem 285: 40699-40705, 2010.

171. Hashimoto M, Majima E, Goto S, Shinohara Y, and Terada $\mathrm{H}$. Fluctuation of the first loop facing the matrix of the mitochondrial ADP/ATP carrier deduced from intermolecular cross-linking of Cys56 residues by bifunctional dimaleimides. Biochemistry 38: 1050-1056, 1999.

172. Herrero A and Barja G. Localization of the site of oxygen radical generation inside the complex I of heart and nonsynaptic brain mammalian mitochondria. J Bioenerg Biomembr 32: 609-615, 2000.

173. Hilfiker-Kleiner D, Hilfiker A, and Drexler H. Many good reasons to have STAT3 in the heart. Pharmacol Ther 107: 131-137, 2005.

174. Hill BG, Higdon AN, Dranka BP, and Darley-Usmar VM. Regulation of vascular smooth muscle cell bioenergetic function by protein glutathiolation. Biochim Biophys Acta 1797: 285-295, 2010.

175. Hirschey MD, Shimazu T, Goetzman E, Jing E, Schwer B, Lombard DB, Grueter CA, Harris C, Biddinger S, Ilkayeva OR, Stevens RD, Li Y, Saha AK, Ruderman NB, Bain JR, Newgard CB, Farese RV, Jr., Alt FW, Kahn CR, and Verdin E. SIRT3 regulates mitochondrial fatty-acid oxidation by reversible enzyme deacetylation. Nature 464: 121-125, 2010. 
176. Hock MB and Kralli A. Transcriptional control of mitochondrial biogenesis and function. Annu Rev Physiol 71: 177-203, 2009.

177. Holmgren A, Johansson C, Berndt C, Lonn ME, Hudemann $\mathrm{C}$, and Lillig $\mathrm{CH}$. Thiol redox control via thioredoxin and glutaredoxin systems. Biochem Soc Trans 33: 1375-1377, 2005.

178. Hu J, Dong $\mathrm{L}$, and Outten CE. The redox environment in the mitochondrial intermembrane space is maintained separately from the cytosol and matrix. J Biol Chem 283: 29126-29134, 2008.

179. Huang X, Ding L, Bennewith KL, Tong RT, Welford SM, Ang KK, Story M, Le QT, and Giaccia AJ. Hypoxia-inducible mir-210 regulates normoxic gene expression involved in tumor initiation. Mol Cell 35: 856-867, 2009.

180. Hurd TR, Filipovska A, Costa NJ, Dahm CC, and Murphy MP. Disulphide formation on mitochondrial protein thiols. Biochem Soc Trans 33: 1390-1393, 2005.

181. Hurd TR, Requejo R, Filipovska A, Brown S, Prime TA, Robinson AJ, Fearnley IM, and Murphy MP. Complex I within oxidatively stressed bovine heart mitochondria is glutathionylated on Cys-531 and Cys-704 of the 75-kDa subunit: potential role of CYS residues in decreasing oxidative damage. J Biol Chem 283: 24801-24815, 2008.

182. Hwang C, Sinskey AJ, and Lodish HF. Oxidized redox state of glutathione in the endoplasmic reticulum. Science 257: 1496-1502, 1992.

183. Hwang JH, Kim DW, Jo EJ, Kim YK, Jo YS, Park JH, Yoo SK, Park MK, Kwak TH, Kho YL, Han J, Choi HS, Lee SH, Kim JM, Lee I, Kyung T, Jang C, Chung J, Kweon GR, and Shong M. Pharmacological stimulation of NADH oxidation ameliorates obesity and related phenotypes in mice. Diabetes 58: 965-974, 2009.

184. Imhoff BR and Hansen JM. Extracellular redox status regulates Nrf2 activation through mitochondrial reactive oxygen species. Biochem J 424: 491-500, 2009.

185. Indran IR, Hande MP, and Pervaiz S. hTERT overexpression alleviates intracellular ROS production, improves mitochondrial function, and inhibits ROS-mediated apoptosis in cancer cells. Cancer Res 71: 266-276, 2011.

186. Iozef R, Becker K, Boehme CC, Schirmer RH, and Werner D. Assembly and functional expression of murine glutathione reductase cDNA: a sequence missing in expressed sequence tag libraries. Biochim Biophys Acta 1500: 137-141, 2000.

187. Isaacs JS, Jung YJ, Mole DR, Lee S, Torres-Cabala C, Chung YL, Merino M, Trepel J, Zbar B, Toro J, Ratcliffe PJ, Linehan WM, and Neckers L. HIF overexpression correlates with biallelic loss of fumarate hydratase in renal cancer: novel role of fumarate in regulation of HIF stability. Cancer Cell 8: 143-153, 2005.

188. Ischiropoulos H. Biological selectivity and functional aspects of protein tyrosine nitration. Biochem Biophys Res Commun 305: 776-783, 2003.

189. Ito Y, Mishra NC, Yoshida K, Kharbanda S, Saxena S, and Kufe D. Mitochondrial targeting of JNK/SAPK in the phorbol ester response of myeloid leukemia cells. Cell Death Differ 8: 794-800, 2001.

190. Iuchi Y, Okada F, Takamiya R, Kibe N, Tsunoda S, Nakajima O, Toyoda K, Nagae R, Suematsu M, Soga T, Uchida K, and Fujii J. Rescue of anaemia and autoimmune responses in SOD1-deficient mice by transgenic expression of human SOD1 in erythrocytes. Biochem J 422: 313-320, 2009.
191. Jaffrey SR, Erdjument-Bromage H, Ferris CD, Tempst P, and Snyder SH. Protein S-nitrosylation: a physiological signal for neuronal nitric oxide. Nat Cell Biol 3: 193-197, 2001.

192. Jiang S, Moriarty-Craige SE, Orr M, Cai J, Sternberg P, Jr., and Jones DP. Oxidant-induced apoptosis in human retinal pigment epithelial cells: dependence on extracellular redox state. Invest Ophthalmol Vis Sci 46: 1054-1061, 2005.

193. Jin RC, Mahoney CE, Coleman Anderson L, Ottaviano F, Croce K, Leopold JA, Zhang YY, Tang SS, Handy DE, and Loscalzo J. Glutathione peroxidase-3 deficiency promotes platelet-dependent thrombosis in vivo. Circulation 123: 1963-1973, 2011.

194. Johansson C, Kavanagh KL, Gileadi O, and Oppermann U. Reversible sequestration of active site cysteines in a $2 \mathrm{Fe}-2 \mathrm{~S}$ bridged dimer provides a mechanism for glutaredoxin 2 regulation in human mitochondria. J Biol Chem 282: 30773082, 2007.

195. Johansson $\mathrm{C}$, Lillig $\mathrm{CH}$, and Holmgren A. Human mitochondrial glutaredoxin reduces S-glutathionylated proteins with high affinity accepting electrons from either glutathione or thioredoxin reductase. J Biol Chem 279: 7537-7543, 2004.

196. Johnson JE, Jr., Choksi K, and Widger WR. NADHUbiquinone oxidoreductase: substrate-dependent oxygen turnover to superoxide anion as a function of flavin mononucleotide. Mitochondrion 3: 97-110, 2003.

197. Jones DP. Redox sensing: orthogonal control in cell cycle and apoptosis signalling. J Intern Med 268: 432-448, 2010.

198. Jones DP, Mody VC, Jr., Carlson JL, Lynn MJ, and Sternberg P, Jr. Redox analysis of human plasma allows separation of pro-oxidant events of aging from decline in antioxidant defenses. Free Radic Biol Med 33: 1290-1300, 2002.

199. Jourd'heuil D, Jourd'heuil FL, and Feelisch M. Oxidation and nitrosation of thiols at low micromolar exposure to nitric oxide. Evidence for a free radical mechanism. J Biol Chem 278: 15720-15726, 2003.

200. Jung $O$, Marklund SL, Geiger H, Pedrazzini T, Busse R, and Brandes RP. Extracellular superoxide dismutase is a major determinant of nitric oxide bioavailability: in vivo and ex vivo evidence from ecSOD-deficient mice. Circ Res 93: 622629, 2003.

201. Kaeberlein M, McVey M, and Guarente L. The SIR2/3/4 complex and SIR2 alone promote longevity in Saccharomyces cerevisiae by two different mechanisms. Genes Dev 13: 2570-2580, 1999.

202. Kaelin WG, Jr. ROS: really involved in oxygen sensing. Cell Metab 1: 357-358, 2005.

203. Kaelin WG, Jr. The von Hippel-Lindau tumour suppressor protein: $\mathrm{O}_{2}$ sensing and cancer. Nat Rev Cancer 8: 865-873, 2008.

204. Kagan VE, Tyurin VA, Jiang J, Tyurina YY, Ritov VB, Amoscato AA, Osipov AN, Belikova NA, Kapralov AA, Kini V, Vlasova, II, Zhao Q, Zou M, Di P, Svistunenko DA, Kurnikov IV, and Borisenko GG. Cytochrome c acts as a cardiolipin oxygenase required for release of proapoptotic factors. Nat Chem Biol 1: 223-232, 2005.

205. Kalinina EV, Chernov NN, and Saprin AN. Involvement of thio-, peroxi-, and glutaredoxins in cellular redoxdependent processes. Biochemistry (Mosc) 73: 1493-1510, 2008.

206. Kamata H, Honda S, Maeda S, Chang L, Hirata H, and Karin M. Reactive oxygen species promote TNFalpha- 
induced death and sustained JNK activation by inhibiting MAP kinase phosphatases. Cell 120: 649-661, 2005.

207. Kamata H, Shibukawa Y, Oka SI, and Hirata H. Epidermal growth factor receptor is modulated by redox through multiple mechanisms. Effects of reductants and $\mathrm{H}_{2} \mathrm{O}_{2}$. Eur J Biochem 267: 1933-1944, 2000.

208. Kanai AJ, Pearce LL, Clemens PR, Birder LA, VanBibber MM, Choi SY, de Groat WC, and Peterson J. Identification of a neuronal nitric oxide synthase in isolated cardiac mitochondria using electrochemical detection. Proc Natl Acad Sci U S A 98: 14126-14131, 2001.

209. Karunakaran S, Saeed U, Ramakrishnan S, Koumar RC, and Ravindranath V. Constitutive expression and functional characterization of mitochondrial glutaredoxin (Grx2) in mouse and human brain. Brain Res 1185: 8-17, 2007.

210. Kelley DE, He J, Menshikova EV, and Ritov VB. Dysfunction of mitochondria in human skeletal muscle in type 2 diabetes. Diabetes 51: 2944-2950, 2002.

211. Kelley EE, Khoo NK, Hundley NJ, Malik UZ, Freeman BA, and Tarpey MM. Hydrogen peroxide is the major oxidant product of xanthine oxidase. Free Radic Biol Med 48: 493498, 2010.

212. Kenet G, Freedman J, Shenkman B, Regina E, Brok-Simoni F, Holzman F, Vavva F, Brand N, Michelson A, Trolliet M, Loscalzo J, and Inbal A. Plasma glutathione peroxidase deficiency and platelet insensitivity to nitric oxide in children with familial stroke. Arterioscler Thromb Vasc Biol 19: 2017-2023, 1999.

213. Kharitonov VG, Sundquist AR, and Sharma VS. Kinetics of nitrosation of thiols by nitric oxide in the presence of oxygen. J Biol Chem 270: 28158-28164, 1995.

214. Kim JW, Tchernyshyov I, Semenza GL, and Dang CV. HIF1-mediated expression of pyruvate dehydrogenase kinase: a metabolic switch required for cellular adaptation to hypoxia. Cell Metab 3: 177-185, 2006.

215. Kimura S, Zhang GX, Nishiyama A, Shokoji T, Yao L, Fan YY, Rahman M, and Abe Y. Mitochondria-derived reactive oxygen species and vascular MAP kinases: comparison of angiotensin II and diazoxide. Hypertension 45: 438-444, 2005.

216. Kirkman HN, Rolfo M, Ferraris AM, and Gaetani GF. Mechanisms of protection of catalase by NADPH. Kinetics and stoichiometry. J Biol Chem 274: 13908-13914, 1999.

217. Klingenberg $\mathrm{M}$ and Winkler $\mathrm{E}$. The reconstituted isolated uncoupling protein is a membrane potential driven $\mathrm{H}+$ translocator. EMBO J 4: 3087-3092, 1985.

218. Knott $\mathrm{AB}$ and Bossy-Wetzel E. Nitric oxide in health and disease of the nervous system. Antioxid Redox Signal 11: 541-554, 2009.

219. Koch-Nolte F, Fischer S, Haag F, and Ziegler M. Compartmentation of $\mathrm{NAD}(+)$-dependent signalling. FEBS Lett 585: 1651-1656, 2011.

220. Koeppen AH. Friedreich's ataxia: pathology, pathogenesis, and molecular genetics. J Neurol Sci 303: 1-12, 2011.

221. Koivunen P, Hirsila M, Remes AM, Hassinen IE, Kivirikko $\mathrm{KI}$, and Myllyharju J. Inhibition of hypoxia-inducible factor (HIF) hydroxylases by citric acid cycle intermediates: possible links between cell metabolism and stabilization of HIF. J Biol Chem 282: 4524-4532, 2007.

222. Kokoszka JE, Waymire KG, Levy SE, Sligh JE, Cai J, Jones DP, MacGregor GR, and Wallace DC. The ADP/ATP translocator is not essential for the mitochondrial permeability transition pore. Nature 427: 461-465, 2004.

223. Koopman WJ, Nijtmans LG, Dieteren CE, Roestenberg P, Valsecchi F, Smeitink JA, and Willems PH. Mammalian mitochondrial complex I: biogenesis, regulation, and reactive oxygen species generation. Antioxid Redox Signal 12: 1431-1470, 2010.

224. Koves TR, Li P, An J, Akimoto T, Slentz D, Ilkayeva O, Dohm GL, Yan Z, Newgard CB, and Muoio DM. Peroxisome proliferator-activated receptor-gamma co-activator 1alpha-mediated metabolic remodeling of skeletal myocytes mimics exercise training and reverses lipid-induced mitochondrial inefficiency. J Biol Chem 280: 33588-33598, 2005.

225. Kowaltowski AJ, Castilho RF, and Vercesi AE. Opening of the mitochondrial permeability transition pore by uncoupling or inorganic phosphate in the presence of $\mathrm{Ca}^{2+}$ is dependent on mitochondrial-generated reactive oxygen species. FEBS Lett 378: 150-152, 1996.

226. Kowaltowski AJ, de Souza-Pinto NC, Castilho RF, and Vercesi AE. Mitochondria and reactive oxygen species. Free Radic Biol Med 47: 333-343, 2009.

227. Kudin AP, Bimpong-Buta NY, Vielhaber S, Elger CE, and Kunz WS. Characterization of superoxide-producing sites in isolated brain mitochondria. J Biol Chem 279: 4127-4135, 2004.

228. Kukidome D, Nishikawa T, Sonoda K, Imoto K, Fujisawa K, Yano M, Motoshima H, Taguchi T, Matsumura T, and Araki E. Activation of AMP-activated protein kinase reduces hyperglycemia-induced mitochondrial reactive oxygen species production and promotes mitochondrial biogenesis in human umbilical vein endothelial cells. Diabetes 55: 120-127, 2006.

229. Kussmaul L and Hirst J. The mechanism of superoxide production by NADH:ubiquinone oxidoreductase (complex I) from bovine heart mitochondria. Proc Natl Acad Sci U S A 103: 7607-7612, 2006.

230. Kyriakis JM and Avruch J. Mammalian mitogen-activated protein kinase signal transduction pathways activated by stress and inflammation. Physiol Rev 81: 807-869, 2001.

231. Lacza Z, Snipes JA, Zhang J, Horvath EM, Figueroa JP, Szabo C, and Busija DW. Mitochondrial nitric oxide synthase is not eNOS, nNOS or iNOS. Free Radic Biol Med 35: 1217-1228, 2003.

232. Lam YW, Yuan Y, Isaac J, Babu CV, Meller J, and Ho SM. Comprehensive identification and modified-site mapping of S-nitrosylated targets in prostate epithelial cells. PLoS One 5: e9075, 2010.

233. Lambert $\mathrm{AJ}$ and Brand $\mathrm{MD}$. Inhibitors of the quinonebinding site allow rapid superoxide production from mitochondrial NADH:ubiquinone oxidoreductase (complex I). J Biol Chem 279: 39414-39420, 2004.

234. Le Moan N, Clement G, Le Maout S, Tacnet F, and Toledano MB. The Saccharomyces cerevisiae proteome of oxidized protein thiols: contrasted functions for the thioredoxin and glutathione pathways. J Biol Chem 281: 1042010430, 2006

235. Le S, Connors TJ, and Maroney AC. c-Jun N-terminal kinase specifically phosphorylates p66ShcA at serine 36 in response to ultraviolet irradiation. J Biol Chem 276: 4833248336, 2001.

236. Lebovitz RM, Zhang H, Vogel H, Cartwright J, Jr., Dionne L, Lu N, Huang S, and Matzuk MM. Neurodegeneration, myocardial injury, and perinatal death in mitochondrial superoxide dismutase-deficient mice. Proc Natl Acad Sci U S A 93: 9782-9787, 1996.

237. Lee HC, Yin PH, Chi CW, and Wei YH. Increase in mitochondrial mass in human fibroblasts under oxidative stress 
and during replicative cell senescence. J Biomed Sci 9: 517526, 2002.

238. Lee HC, Yin PH, Lu CY, Chi CW, and Wei YH. Increase of mitochondria and mitochondrial DNA in response to oxidative stress in human cells. Biochem J $348 \mathrm{Pt} 2$ : 425-432, 2000.

239. Lee TH, Kim SU, Yu SL, Kim SH, Park DS, Moon HB, Dho SH, Kwon KS, Kwon HJ, Han YH, Jeong S, Kang SW, Shin HS, Lee KK, Rhee SG, and Yu DY. Peroxiredoxin II is essential for sustaining life span of erythrocytes in mice. Blood 101: 5033-5038, 2003.

240. Lee TS and Chau LY. Heme oxygenase- 1 mediates the antiinflammatory effect of interleukin-10 in mice. Nat Med 8: 240-246, 2002.

241. Lei C, Niu X, Wei J, Zhu J, and Zhu Y. Interaction of glutathione peroxidase- 1 and selenium in endemic dilated cardiomyopathy. Clin Chim Acta 399: 102-108, 2009.

242. Lemasters JJ, Nieminen AL, Qian T, Trost LC, Elmore SP, Nishimura Y, Crowe RA, Cascio WE, Bradham CA, Brenner DA, and Herman B. The mitochondrial permeability transition in cell death: a common mechanism in necrosis, apoptosis and autophagy. Biochim Biophys Acta 1366: 177196, 1998.

243. Leopold JA and Loscalzo J. Oxidative enzymopathies and vascular disease. Arterioscler Thromb Vasc Biol 25: 13321340, 2005.

244. Leopold JA, Walker J, Scribner AW, Voetsch B, Zhang YY, Loscalzo AJ, Stanton RC, and Loscalzo J. Glucose-6phosphate dehydrogenase modulates vascular endothelial growth factor-mediated angiogenesis. J Biol Chem 278: 32100-32106, 2003.

245. Leopold JA, Zhang YY, Scribner AW, Stanton RC, and Loscalzo J. Glucose-6-phosphate dehydrogenase overexpression decreases endothelial cell oxidant stress and increases bioavailable nitric oxide. Arterioscler Thromb Vasc Biol 23: 411-417, 2003.

246. Lesnefsky EJ, Chen Q, Moghaddas S, Hassan MO, Tandler $\mathrm{B}$, and Hoppel CL. Blockade of electron transport during ischemia protects cardiac mitochondria. J Biol Chem 279: 47961-47967, 2004.

247. Leung AW and Halestrap AP. Recent progress in elucidating the molecular mechanism of the mitochondrial permeability transition pore. Biochim Biophys Acta 1777: 946-952, 2008.

248. Lewis P, Stefanovic N, Pete J, Calkin AC, Giunti S, ThallasBonke V, Jandeleit-Dahm KA, Allen TJ, Kola I, Cooper ME, and de Haan JB. Lack of the antioxidant enzyme glutathione peroxidase- 1 accelerates atherosclerosis in diabetic apolipoprotein E-deficient mice. Circulation 115: 2178-2187, 2007.

249. Li JM and Shah AM. Endothelial cell superoxide generation: regulation and relevance for cardiovascular pathophysiology. Am J Physiol Regul Integr Comp Physiol 287: R1014-R1030, 2004.

250. Li M, Chiou KR, and Kass DA. Shear stress inhibition of $\mathrm{H}(2) \mathrm{O}(2)$ induced p66(Shc) phosphorylation by ASK1-JNK inactivation in endothelium. Heart Vessels 22: 423-427, 2007.

251. Li P, Nijhawan D, Budihardjo I, Srinivasula SM, Ahmad M, Alnemri ES, and Wang X. Cytochrome $\mathrm{c}$ and dATPdependent formation of Apaf-1/caspase-9 complex initiates an apoptotic protease cascade. Cell 91: 479-489, 1997.

252. Li Y, Huang TT, Carlson EJ, Melov S, Ursell PC, Olson JL, Noble LJ, Yoshimura MP, Berger C, Chan PH, Wallace DC, and Epstein CJ. Dilated cardiomyopathy and neonatal lethality in mutant mice lacking manganese superoxide dismutase. Nat Genet 11: 376-381, 1995.

253. Liang H, Ran Q, Jang YC, Holstein D, Lechleiter J, McDonaldMarsh T, Musatov A, Song W, Van Remmen H, and Richardson A. Glutathione peroxidase 4 differentially regulates the release of apoptogenic proteins from mitochondria. Free Radic Biol Med 47: 312-320, 2009.

254. Liddell JR, Dringen R, Crack PJ, and Robinson SR. Glutathione peroxidase 1 and a high cellular glutathione concentration are essential for effective organic hydroperoxide detoxification in astrocytes. Glia 54: 873-879, 2006.

255. Lillig $\mathrm{CH}$, Lonn $\mathrm{ME}$, Enoksson $\mathrm{M}$, Fernandes $\mathrm{AP}$, and Holmgren A. Short interfering RNA-mediated silencing of glutaredoxin 2 increases the sensitivity of HeLa cells toward doxorubicin and phenylarsine oxide. Proc Natl Acad Sci U S A 101: 13227-13232, 2004.

256. Lim CC, Bryan NS, Jain M, Garcia-Saura MF, Fernandez BO, Sawyer DB, Handy DE, Loscalzo J, Feelisch M, and Liao R. Glutathione peroxidase deficiency exacerbates ischemia-reperfusion injury in male but not female myocardium: insights into antioxidant compensatory mechanisms. Am J Physiol Heart Circ Physiol 297: H2144-H2153, 2009.

257. Lin TK, Hughes G, Muratovska A, Blaikie FH, Brookes PS, Darley-Usmar V, Smith RA, and Murphy MP. Specific modification of mitochondrial protein thiols in response to oxidative stress: a proteomics approach. J Biol Chem 277: 17048-17056, 2002.

258. Linard D, Kandlbinder A, Degand H, Morsomme $\mathrm{P}$, Dietz KJ, and Knoops B. Redox characterization of human cyclophilin D: identification of a new mammalian mitochondrial redox sensor? Arch Biochem Biophys 491: 39-45, 2009.

259. Linares GR, Xing W, Govoni KE, Chen ST, and Mohan S. Glutaredoxin 5 regulates osteoblast apoptosis by protecting against oxidative stress. Bone 44: 795-804, 2009.

260. Liochev SI and Fridovich I. The effects of superoxide dismutase on $\mathrm{H}_{2} \mathrm{O}_{2}$ formation. Free Radic Biol Med 42: 1465$1469,2007$.

261. Liu $\mathrm{T}$ and $\mathrm{O}^{\prime}$ Rourke B. Regulation of mitochondrial $\mathrm{Ca}^{2+}$ and its effects on energetics and redox balance in normal and failing heart. J Bioenerg Biomembr 41: 127-132, 2009.

262. Liu Y, Fiskum G, and Schubert D. Generation of reactive oxygen species by the mitochondrial electron transport chain. J Neurochem 80: 780-787, 2002.

263. Liu Z, Rudd MA, Freedman JE, and Loscalzo J. S-Transnitrosation reactions are involved in the metabolic fate and biological actions of nitric oxide. J Pharmacol Exp Ther 284: 526-534, 1998.

264. Loh K, Deng H, Fukushima A, Cai X, Boivin B, Galic S, Bruce C, Shields BJ, Skiba B, Ooms LM, Stepto N, Wu B, Mitchell CA, Tonks NK, Watt MJ, Febbraio MA, Crack PJ, Andrikopoulos S, and Tiganis T. Reactive oxygen species enhance insulin sensitivity. Cell Metab 10: 260-272, 2009.

265. Lubos E, Loscalzo J, and Handy DE. Glutathione peroxidase-1 in health and disease: from molecular mechanisms to therapeutic opportunities. Antioxid Redox Signal 15: 1957-1997, 2011.

266. Lund DD, Chu Y, Miller JD, and Heistad DD. Protective effect of extracellular superoxide dismutase on endothelial function during aging. Am J Physiol Heart Circ Physiol 296: H1920-H1925, 2009.

267. Lundberg $M$, Johansson C, Chandra J, Enoksson M, Jacobsson G, Ljung J, Johansson M, and Holmgren A. Cloning and expression of a novel human glutaredoxin 
(Grx2) with mitochondrial and nuclear isoforms. J Biol Chem 276: 26269-26275, 2001.

268. Maciel EN, Vercesi AE, and Castilho RF. Oxidative stress in $\mathrm{Ca}(2+)$-induced membrane permeability transition in brain mitochondria. J Neurochem 79: 1237-1245, 2001.

269. Maehama T and Dixon JE. The tumor suppressor, PTEN/ MMAC1, dephosphorylates the lipid second messenger, phosphatidylinositol 3,4,5-trisphosphate. J Biol Chem 273: 13375-13378, 1998.

270. Maeng O, Kim YC, Shin HJ, Lee JO, Huh TL, Kang KI, Kim YS, Paik SG, and Lee H. Cytosolic NADP(+)-dependent isocitrate dehydrogenase protects macrophages from LPSinduced nitric oxide and reactive oxygen species. Biochem Biophys Res Commun 317: 558-564, 2004.

271. Mailloux RJ, Seifert EL, Bouillaud F, Aguer C, Collins S, and Harper ME. Glutathionylation acts as a control switch for uncoupling proteins UCP2 and UCP3. J Biol Chem 286: 21865-21875, 2011.

272. Maines MD. The heme oxygenase system: update 2005. Antioxid Redox Signal 7: 1761-1766, 2005.

273. Manevich Y and Fisher AB. Peroxiredoxin 6, a 1-Cys peroxiredoxin, functions in antioxidant defense and lung phospholipid metabolism. Free Radic Biol Med 38: 14221432, 2005.

274. Mansfield KD, Guzy RD, Pan Y, Young RM, Cash TP, Schumacker PT, and Simon MC. Mitochondrial dysfunction resulting from loss of cytochrome c impairs cellular oxygen sensing and hypoxic HIF-alpha activation. Cell Metab 1: 393-399, 2005.

275. Mari M, Morales A, Colell A, Garcia-Ruiz C, and Fernandez-Checa JC. Mitochondrial glutathione, a key survival antioxidant. Antioxid Redox Signal 11: 2685-2700, 2009.

276. Markert CL. Lactate dehydrogenase. Biochemistry and function of lactate dehydrogenase. Cell Biochem Funct 2: 131-134, 1984.

277. Martin LJ, Adams NA, Pan Y, Price A, and Wong M. The mitochondrial permeability transition pore regulates nitric oxide-mediated apoptosis of neurons induced by target deprivation. J Neurosci 31: 359-370, 2011.

278. Martinez JI, Garcia RD, and Galarza AM. The kinetic mechanism of glutathione peroxidase from human platelets. Thromb Res 27: 197-203, 1982.

279. Masson N, Willam C, Maxwell PH, Pugh CW, and Ratcliffe PJ. Independent function of two destruction domains in hypoxia-inducible factor-alpha chains activated by prolyl hydroxylation. EMBO J 20: 5197-5206, 2001.

280. Matsui M, Oshima M, Oshima H, Takaku K, Maruyama T, Yodoi J, and Taketo MM. Early embryonic lethality caused by targeted disruption of the mouse thioredoxin gene. Dev Biol 178: 179-185, 1996.

281. Matsunaga T, Kotamraju S, Kalivendi SV, Dhanasekaran A, Joseph J, and Kalyanaraman B. Ceramide-induced intracellular oxidant formation, iron signaling, and apoptosis in endothelial cells: protective role of endogenous nitric oxide. J Biol Chem 279: 28614-28624, 2004.

282. Matsuzawa A and Ichijo H. Redox control of cell fate by MAP kinase: physiological roles of ASK1-MAP kinase pathway in stress signaling. Biochim Biophys Acta 1780: 1325-1336, 2008.

283. Matsuzawa A, Saegusa K, Noguchi T, Sadamitsu C, Nishitoh H, Nagai S, Koyasu S, Matsumoto K, Takeda K, and Ichijo H. ROS-dependent activation of the TRAF6-ASK1p38 pathway is selectively required for TLR4-mediated innate immunity. Nat Immunol 6: 587-592, 2005.
284. McClung JP, Roneker CA, Mu W, Lisk DJ, Langlais P, Liu $\mathrm{F}$, and Lei XG. Development of insulin resistance and obesity in mice overexpressing cellular glutathione peroxidase. Proc Natl Acad Sci U S A 101: 8852-8857, 2004.

285. McLeod CJ, Aziz A, Hoyt RF, Jr., McCoy JP, Jr., and Sack MN. Uncoupling proteins 2 and 3 function in concert to augment tolerance to cardiac ischemia. J Biol Chem 280: 33470-33476, 2005.

286. McStay GP, Clarke SJ, and Halestrap AP. Role of critical thiol groups on the matrix surface of the adenine nucleotide translocase in the mechanism of the mitochondrial permeability transition pore. Biochem J 367: 541-548, 2002.

287. Meister A. Mitochondrial changes associated with glutathione deficiency. Biochim Biophys Acta 1271: 35-42, 1995.

288. Meister A and Tate SS. Glutathione and related gammaglutamyl compounds: biosynthesis and utilization. Annu Rev Biochem 45: 559-604, 1976.

289. Mejias R, Villadiego J, Pintado CO, Vime PJ, Gao L, ToledoAral JJ, Echevarria M, and Lopez-Barneo J. Neuroprotection by transgenic expression of glucose-6-phosphate dehydrogenase in dopaminergic nigrostriatal neurons of mice. J Neurosci 26: 4500-4508, 2006.

290. Melov S. Mitochondrial oxidative stress. Physiologic consequences and potential for a role in aging. Ann N Y Acad Sci 908: 219-225, 2000.

291. Menini S, Amadio L, Oddi G, Ricci C, Pesce C, Pugliese F, Giorgio M, Migliaccio E, Pelicci P, Iacobini C, and Pugliese G. Deletion of p66Shc longevity gene protects against experimental diabetic glomerulopathy by preventing diabetes-induced oxidative stress. Diabetes 55: 1642-1650, 2006.

292. Mesecke N, Terziyska N, Kozany C, Baumann F, Neupert W, Hell K, and Herrmann JM. A disulfide relay system in the intermembrane space of mitochondria that mediates protein import. Cell 121: 1059-1069, 2005.

293. Michael LF, Wu Z, Cheatham RB, Puigserver P, Adelmant G, Lehman JJ, Kelly DP, and Spiegelman BM. Restoration of insulin-sensitive glucose transporter (GLUT4) gene expression in muscle cells by the transcriptional coactivator PGC-1. Proc Natl Acad Sci U S A 98: 3820-3825, 2001.

294. Michel S, Wanet A, De Pauw A, Rommelaere G, Arnould $\mathrm{T}$, and Renard P. Crosstalk between mitochondrial (dys)function and mitochondrial abundance. J Cell Physiol 2011 [Epub ahead of print]; DOI: 10.1002/jcp.23021.

295. Michishita E, Park JY, Burneskis JM, Barrett JC, and Horikawa I. Evolutionarily conserved and nonconserved cellular localizations and functions of human SIRT proteins. Mol Biol Cell 16: 4623-4635, 2005.

296. Mieyal JJ, Gallogly MM, Qanungo S, Sabens EA, and Shelton MD. Molecular mechanisms and clinical implications of reversible protein S-glutathionylation. Antioxid Redox Signal 10: 1941-1988, 2008.

297. Migliaccio E, Giorgio M, Mele S, Pelicci G, Reboldi P, Pandolfi PP, Lanfrancone L, and Pelicci PG. The p66shc adaptor protein controls oxidative stress response and life span in mammals. Nature 402: 309-313, 1999.

298. Mills GC. Hemoglobin catabolism. I. Glutathione peroxidase, an erythrocyte enzyme which protects hemoglobin from oxidative breakdown. J Biol Chem 229: 189-197, 1957.

299. Molina-Navarro MM, Casas C, Piedrafita L, Belli G, and Herrero E. Prokaryotic and eukaryotic monothiol glutaredoxins are able to perform the functions of Grx5 in the biogenesis of Fe/S clusters in yeast mitochondria. FEBS Lett 580: 2273-2280, 2006. 
300. Monteiro P, Duarte AI, Goncalves LM, and Providencia LA. Valsartan improves mitochondrial function in hearts submitted to acute ischemia. Eur J Pharmacol 518: 158-164, 2005.

301. Mookerjee SA, Divakaruni AS, Jastroch M, and Brand MD. Mitochondrial uncoupling and lifespan. Mech Ageing Dev 131: 463-472, 2010.

302. Moriarty SE, Shah JH, Lynn M, Jiang S, Openo K, Jones DP, and Sternberg P. Oxidation of glutathione and cysteine in human plasma associated with smoking. Free Radic Biol Med 35: 1582-1588, 2003.

303. Muller FL, Liu Y, Abdul-Ghani MA, Lustgarten MS, Bhattacharya A, Jang YC, and Van Remmen H. High rates of superoxide production in skeletal-muscle mitochondria respiring on both complex I- and complex II-linked substrates. Biochem J 409: 491-499, 2008.

304. Murphy MP. How mitochondria produce reactive oxygen species. Biochem J 417: 1-13, 2009.

305. Murray CI, Kane LA, Uhrigshardt H, Wang SB, and Van Eyk JE. Site-mapping of in vitro S-nitrosation in cardiac mitochondria: implications for cardioprotection. Mol Cell Proteomics 10: M110 004721, 2010.

306. Nadtochiy SM, Burwell LS, Ingraham CA, Spencer CM, Friedman AE, Pinkert CA, and Brookes PS. In vivo cardioprotection by S-nitroso-2-mercaptopropionyl glycine. J Mol Cell Cardiol 46: 960-968, 2009.

307. Nadtochiy SM, Tompkins AJ, and Brookes PS. Different mechanisms of mitochondrial proton leak in ischaemia/ reperfusion injury and preconditioning: implications for pathology and cardioprotection. Biochem J 395: 611-618, 2006.

308. Nagy N, Malik G, Fisher AB, and Das DK. Targeted disruption of peroxiredoxin 6 gene renders the heart vulnerable to ischemia-reperfusion injury. Am J Physiol Heart Circ Physiol 291: H2636-H2640, 2006.

309. Nakagawa T, Shimizu S, Watanabe T, Yamaguchi O, Otsu $\mathrm{K}$, Yamagata $\mathrm{H}$, Inohara $\mathrm{H}, \mathrm{Kubo} \mathrm{T}$, and Tsujimoto $\mathrm{Y}$. Cyclophilin D-dependent mitochondrial permeability transition regulates some necrotic but not apoptotic cell death. Nature 434: 652-658, 2005.

310. Nakagawa Y. Initiation of apoptotic signal by the peroxidation of cardiolipin of mitochondria. Ann N Y Acad Sci 1011: 177-184, 2004.

311. Narendra D, Tanaka A, Suen DF, and Youle RJ. Parkin is recruited selectively to impaired mitochondria and promotes their autophagy. J Cell Biol 183: 795-803, 2008.

312. Nedospasov A, Rafikov R, Beda N, and Nudler E. An autocatalytic mechanism of protein nitrosylation. Proc Natl Acad Sci U S A 97: 13543-13548, 2000.

313. Neumann CA, Krause DS, Carman CV, Das S, Dubey DP, Abraham JL, Bronson RT, Fujiwara Y, Orkin SH, and Van Etten RA. Essential role for the peroxiredoxin Prdx1 in erythrocyte antioxidant defence and tumour suppression. Nature 424: 561-565, 2003.

314. Nicholls DG and Locke RM. Thermogenic mechanisms in brown fat. Physiol Rev 64: 1-64, 1984.

315. Nissen SE and Wolski K. Effect of rosiglitazone on the risk of myocardial infarction and death from cardiovascular causes. N Engl J Med 356: 2457-2471, 2007.

316. Nomura K, Imai H, Koumura T, Arai M, and Nakagawa Y. Mitochondrial phospholipid hydroperoxide glutathione peroxidase suppresses apoptosis mediated by a mitochondrial death pathway. J Biol Chem 274: 29294-29302, 1999.
317. Nonn L, Williams RR, Erickson RP, and Powis G. The absence of mitochondrial thioredoxin 2 causes massive apoptosis, exencephaly, and early embryonic lethality in homozygous mice. Mol Cell Biol 23: 916-922, 2003.

318. Okado-Matsumoto A and Fridovich I. Subcellular distribution of superoxide dismutases (SOD) in rat liver: $\mathrm{Cu}, \mathrm{Zn}$ SOD in mitochondria. J Biol Chem 276: 38388-38393, 2001.

319. Ostergaard H, Tachibana C, and Winther JR. Monitoring disulfide bond formation in the eukaryotic cytosol. J Cell Biol 166: 337-345, 2004.

320. Pan Y, Mansfield KD, Bertozzi CC, Rudenko V, Chan DA, Giaccia AJ, and Simon MC. Multiple factors affecting cellular redox status and energy metabolism modulate hypoxia-inducible factor prolyl hydroxylase activity in vivo and in vitro. Mol Cell Biol 27: 912-925, 2007.

321. Paradies G, Petrosillo G, Paradies V, and Ruggiero FM. Role of cardiolipin peroxidation and $\mathrm{Ca}^{2+}$ in mitochondrial dysfunction and disease. Cell Calcium 45: 643-650, 2009.

322. Passos JF, Saretzki G, and von Zglinicki T. DNA damage in telomeres and mitochondria during cellular senescence: is there a connection? Nucleic Acids Res 35: 7505-7513, 2007.

323. Paulsen CE and Carroll KS. Orchestrating redox signaling networks through regulatory cysteine switches. ACS Chem Biol 5: 47-62, 2010.

324. Perron NR and Brumaghim JL. A review of the antioxidant mechanisms of polyphenol compounds related to iron binding. Cell Biochem Biophys 53: 75-100, 2009.

325. Petersen KF, Befroy D, Dufour S, Dziura J, Ariyan C, Rothman DL, DiPietro L, Cline GW, and Shulman GI. Mitochondrial dysfunction in the elderly: possible role in insulin resistance. Science 300: 1140-1142, 2003.

326. Petrosillo G, Di Venosa N, Pistolese M, Casanova G, Tiravanti E, Colantuono G, Federici A, Paradies G, and Ruggiero FM. Protective effect of melatonin against mitochondrial dysfunction associated with cardiac ischemia- reperfusion: role of cardiolipin. FASEB J 20: 269276, 2006.

327. Pfeiffer K, Gohil V, Stuart RA, Hunte C, Brandt U, Greenberg ML, and Schagger $\mathrm{H}$. Cardiolipin stabilizes respiratory chain supercomplexes. J Biol Chem 278: 52873-52880, 2003.

328. Pi J, Bai Y, Daniel KW, Liu D, Lyght O, Edelstein D, Brownlee M, Corkey BE, and Collins S. Persistent oxidative stress due to absence of uncoupling protein 2 associated with impaired pancreatic beta-cell function. Endocrinology 150: 3040-3048, 2009.

329. Piantadosi CA, Carraway MS, Babiker A, and Suliman HB. Heme oxygenase- 1 regulates cardiac mitochondrial biogenesis via Nrf2-mediated transcriptional control of nuclear respiratory factor-1. Circ Res 103: 1232-1240, 2008.

330. Piantadosi CA and Suliman HB. Mitochondrial transcription factor A induction by redox activation of nuclear respiratory factor 1. J Biol Chem 281: 324-333, 2006.

331. Pigeolet E, Corbisier P, Houbion A, Lambert D, Michiels C, Raes M, Zachary MD, and Remacle J. Glutathione peroxidase, superoxide dismutase, and catalase inactivation by peroxides and oxygen derived free radicals. Mech Ageing Dev 51: 283-297, 1990.

332. Pink JJ, Planchon SM, Tagliarino C, Varnes ME, Siegel D, and Boothman DA. NAD(P)H:Quinone oxidoreductase activity is the principal determinant of beta-lapachone cytotoxicity. J Biol Chem 275: 5416-5424, 2000.

333. Planson AG, Palais G, Abbas K, Gerard M, Couvelard L, Delaunay A, Baulande S, Drapier JC, and Toledano MB. 
Sulfiredoxin protects mice from lipopolysaccharideinduced endotoxic shock. Antioxid Redox Signal 14: 20712080, 2011.

334. Pollard PJ, Briere JJ, Alam NA, Barwell J, Barclay E, Wortham NC, Hunt T, Mitchell M, Olpin S, Moat SJ, Hargreaves IP, Heales SJ, Chung YL, Griffiths JR, Dalgleish A, McGrath JA, Gleeson MJ, Hodgson SV, Poulsom R, Rustin P, and Tomlinson IP. Accumulation of Krebs cycle intermediates and over-expression of HIF1alpha in tumours which result from germline $\mathrm{FH}$ and SDH mutations. Hum Mol Genet 14: 2231-2239, 2005.

335. Pretsch W. Enzyme-activity mutants in Mus musculus. I. Phenotypic description and genetic characterization of ethylnitrosourea-induced mutations. Mamm Genome 11: 537-542, 2000.

336. Prime TA, Blaikie FH, Evans C, Nadtochiy SM, James AM, Dahm CC, Vitturi DA, Patel RP, Hiley CR, Abakumova I, Requejo R, Chouchani ET, Hurd TR, Garvey JF, Taylor CT, Brookes PS, Smith RA, and Murphy MP. A mitochondria-targeted S-nitrosothiol modulates respiration, nitrosates thiols, and protects against ischemiareperfusion injury. Proc Natl Acad Sci U S A 106: 1076410769, 2009.

337. Puigserver $\mathrm{P}, \mathrm{Wu} \mathrm{Z}$, Park CW, Graves R, Wright $\mathrm{M}$, and Spiegelman BM. A cold-inducible coactivator of nuclear receptors linked to adaptive thermogenesis. Cell 92: 829839, 1998.

338. Rabilloud T, Heller M, Gasnier F, Luche S, Rey C, Aebersold R, Benahmed M, Louisot P, and Lunardi J. Proteomics analysis of cellular response to oxidative stress. Evidence for in vivo overoxidation of peroxiredoxins at their active site. J Biol Chem 277: 19396-19401, 2002.

339. Radi R, Turrens JF, Chang LY, Bush KM, Crapo JD, and Freeman BA. Detection of catalase in rat heart mitochondria. J Biol Chem 266: 22028-22034, 1991.

340. Ran Q, Liang H, Gu M, Qi W, Walter CA, Roberts LJ, 2nd, Herman B, Richardson A, and Van Remmen H. Transgenic mice overexpressing glutathione peroxidase 4 are protected against oxidative stress-induced apoptosis. J Biol Chem 279: 55137-55146, 2004.

341. Randle PJ. Regulatory interactions between lipids and carbohydrates: the glucose fatty acid cycle after 35 years. Diabetes Metab Rev 14: 263-283, 1998.

342. Rasbach KA and Schnellmann RG. Signaling of mitochondrial biogenesis following oxidant injury. J Biol Chem 282: 2355-2362, 2007.

343. Raval RR, Lau KW, Tran MG, Sowter HM, Mandriota SJ, Li JL, Pugh CW, Maxwell PH, Harris AL, and Ratcliffe PJ. Contrasting properties of hypoxia-inducible factor 1 (HIF-1) and HIF-2 in von Hippel-Lindau-associated renal cell carcinoma. Mol Cell Biol 25: 5675-5686, 2005.

344. Reeves MA and Hoffmann PR. The human selenoproteome: recent insights into functions and regulation. Cell Mol Life Sci 66: 2457-2478, 2009.

345. Rehder DS and Borges CR. Cysteine sulfenic acid as an intermediate in disulfide bond formation and nonenzymatic protein folding. Biochemistry 49: 7748-7755, 2010.

346. Rhee SG, Chae HZ, and Kim K. Peroxiredoxins: a historical overview and speculative preview of novel mechanisms and emerging concepts in cell signaling. Free Radic Biol Med 38: 1543-1552, 2005.

347. Riemer J, Fischer M, and Herrmann JM. Oxidation-driven protein import into mitochondria: Insights and blind spots. Biochim Biophys Acta 1808: 981-989, 2011.
348. Rigobello MP, Callegaro MT, Barzon E, Benetti M, and Bindoli A. Purification of mitochondrial thioredoxin reductase and its involvement in the redox regulation of membrane permeability. Free Radic Biol Med 24: 370-376, 1998.

349. Rigoulet M, Yoboue ED, and Devin A. Mitochondrial ROS generation and its regulation: mechanisms involved in $\mathrm{H}(2) \mathrm{O}(2)$ signaling. Antioxid Redox Signal 14: 459-468, 2011.

350. Ristow $M$ and Zarse K. How increased oxidative stress promotes longevity and metabolic health: the concept of mitochondrial hormesis (mitohormesis). Exp Gerontol 45: 410-418, 2010.

351. Ritov VB, Menshikova EV, Azuma K, Wood R, Toledo FG, Goodpaster BH, Ruderman NB, and Kelley DE. Deficiency of electron transport chain in human skeletal muscle mitochondria in type 2 diabetes mellitus and obesity. Am J Physiol Endocrinol Metab 298: E49-E58, 2010.

352. Rizzuto R, Pinton P, Carrington W, Fay FS, Fogarty KE, Lifshitz LM, Tuft RA, and Pozzan T. Close contacts with the endoplasmic reticulum as determinants of mitochondrial $\mathrm{Ca}^{2+}$ responses. Science 280: 1763-1766, 1998.

353. Robinson NC. Functional binding of cardiolipin to cytochrome c oxidase. J Bioenerg Biomembr 25: 153-163, 1993.

354. Rodgers JT, Lerin C, Haas W, Gygi SP, Spiegelman BM, and Puigserver P. Nutrient control of glucose homeostasis through a complex of PGC-1alpha and SIRT1. Nature 434: 113-118, 2005.

355. Rodriguez-Manzaneque MT, Tamarit J, Belli G, Ros J, and Herrero E. Grx5 is a mitochondrial glutaredoxin required for the activity of iron/sulfur enzymes. Mol Biol Cell 13: 1109-1121, 2002.

356. Rossig L, Fichtlscherer B, Breitschopf K, Haendeler J, Zeiher AM, Mulsch A, and Dimmeler S. Nitric oxide inhibits caspase-3 by S-nitrosation in vivo. J Biol Chem 274: 68236826, 1999.

357. Rota M, LeCapitaine N, Hosoda T, Boni A, De Angelis A, Padin-Iruegas ME, Esposito G, Vitale S, Urbanek K, Casarsa C, Giorgio M, Luscher TF, Pelicci PG, Anversa P, Leri A, and Kajstura J. Diabetes promotes cardiac stem cell aging and heart failure, which are prevented by deletion of the p66shc gene. Circ Res 99: 42-52, 2006.

358. Rotruck JT, Pope AL, Ganther HE, and Hoekstra WG. Prevention of oxidative damage to rat erythrocytes by dietary selenium. J Nutr 102: 689-696, 1972.

359. Rotruck JT, Pope AL, Ganther HE, Swanson AB, Hafeman DG, and Hoekstra WG. Selenium: biochemical role as a component of glutathione peroxidase. Science 179: 588-590, 1973.

360. Rouault TA and Tong WH. Iron-sulfur cluster biogenesis and human disease. Trends Genet 24: 398-407, 2008.

361. Rydstrom J. Mitochondrial NADPH, transhydrogenase and disease. Biochim Biophys Acta 1757: 721-726, 2006.

362. Ryu SY, Beutner G, Dirksen RT, Kinnally KW, and Sheu SS. Mitochondrial ryanodine receptors and other mitochondrial $\mathrm{Ca}^{2+}$ permeable channels. FEBS Lett 584: 1948-1955, 2010.

363. Saeed U, Durgadoss L, Valli RK, Joshi DC, Joshi PG, and Ravindranath V. Knockdown of cytosolic glutaredoxin 1 leads to loss of mitochondrial membrane potential: implication in neurodegenerative diseases. PLoS One 3: e2459, 2008.

364. Sahin E, Colla S, Liesa M, Moslehi J, Muller FL, Guo M, Cooper M, Kotton D, Fabian AJ, Walkey C, Maser RS, Tonon G, Foerster F, Xiong R, Wang YA, Shukla SA, 
Jaskelioff M, Martin ES, Heffernan TP, Protopopov A, Ivanova E, Mahoney JE, Kost-Alimova M, Perry SR, Bronson R, Liao R, Mulligan R, Shirihai OS, Chin L, and DePinho RA. Telomere dysfunction induces metabolic and mitochondrial compromise. Nature 470: 359-365, 2011.

365. Saitoh M, Nishitoh H, Fujii M, Takeda K, Tobiume K, Sawada Y, Kawabata M, Miyazono K, and Ichijo H. Mammalian thioredoxin is a direct inhibitor of apoptosis signal-regulating kinase (ASK) 1. EMBO J 17: 2596-2606, 1998.

366. Salerno JC, Harris DE, Irizarry K, Patel B, Morales AJ, Smith SM, Martasek P, Roman LJ, Masters BS, Jones CL, Weissman BA, Lane P, Liu Q, and Gross SS. An autoinhibitory control element defines calcium-regulated isoforms of nitric oxide synthase. J Biol Chem 272: 2976929777, 1997.

367. Salopuro T, Pulkkinen L, Lindstrom J, Kolehmainen M, Tolppanen AM, Eriksson JG, Valle TT, Aunola S, IlanneParikka P, Keinanen-Kiukaanniemi S, Tuomilehto J, Laakso $\mathrm{M}$, and Uusitupa M. Variation in the UCP2 and UCP3 genes associates with abdominal obesity and serum lipids: the Finnish Diabetes Prevention Study. BMC Med Genet 10: 94, 2009.

368. Salsbury FR, Jr., Knutson ST, Poole LB, and Fetrow JS. Functional site profiling and electrostatic analysis of cysteines modifiable to cysteine sulfenic acid. Protein Sci 17: 299-312, 2008.

369. Salvi M, Battaglia V, Brunati AM, La Rocca N, Tibaldi E, Pietrangeli P, Marcocci L, Mondovi B, Rossi CA, and Toninello A. Catalase takes part in rat liver mitochondria oxidative stress defense. J Biol Chem 282: 24407-24415, 2007.

370. Santo-Domingo J and Demaurex N. Calcium uptake mechanisms of mitochondria. Biochim Biophys Acta 1797: 907-912, 2010.

371. Sauvanet C, Duvezin-Caubet S, di Rago JP, and Rojo M. Energetic requirements and bioenergetic modulation of mitochondrial morphology and dynamics. Semin Cell Dev Biol 21: 558-565, 2010.

372. Savvides SN, Scheiwein M, Bohme CC, Arteel GE, Karplus PA, Becker K, and Schirmer RH. Crystal structure of the antioxidant enzyme glutathione reductase inactivated by peroxynitrite. J Biol Chem 277: 2779-2784, 2002.

373. Scarpulla RC. Nuclear activators and coactivators in mammalian mitochondrial biogenesis. Biochim Biophys Acta 1576: 1-14, 2002.

374. Schafer FQ and Buettner GR. Redox environment of the cell as viewed through the redox state of the glutathione disulfide/glutathione couple. Free Radic Biol Med 30: 11911212, 2001.

375. Scherz-Shouval R, Shvets E, Fass E, Shorer H, Gil L, and Elazar Z. Reactive oxygen species are essential for autophagy and specifically regulate the activity of Atg4. EMBO J 26: 1749-1760, 2007.

376. Schipper HM. Heme oxygenase-1: role in brain aging and neurodegeneration. Exp Gerontol 35: 821-830, 2000.

377. Schnabel R, Lackner KJ, Rupprecht HJ, Espinola-Klein C, Torzewski M, Lubos E, Bickel C, Cambien F, Tiret L, Munzel T, and Blankenberg S. Glutathione peroxidase-1 and homocysteine for cardiovascular risk prediction: results from the AtheroGene study. J Am Coll Cardiol 45: 1631-1637, 2005.

378. Schnurr K, Belkner J, Ursini F, Schewe T, and Kuhn H. The selenoenzyme phospholipid hydroperoxide glutathione peroxidase controls the activity of the 15-lipoxygenase with complex substrates and preserves the specificity of the oxygenation products. J Biol Chem 271: 4653-4658, 1996.

379. Schonfeld P, Wieckowski MR, Lebiedzinska M, and Wojtczak L. Mitochondrial fatty acid oxidation and oxidative stress: lack of reverse electron transfer-associated production of reactive oxygen species. Biochim Biophys Acta 1797: 929-938, 2010.

380. Schreiber SN, Emter R, Hock MB, Knutti D, Cardenas J, Podvinec M, Oakeley EJ, and Kralli A. The estrogenrelated receptor alpha (ERRalpha) functions in PPARgamma coactivator 1alpha (PGC-1alpha)-induced mitochondrial biogenesis. Proc Natl Acad Sci U S A 101: 6472-6477, 2004.

381. Schroder E, Brennan JP, and Eaton P. Cardiac peroxiredoxins undergo complex modifications during cardiac oxidant stress. Am J Physiol Heart Circ Physiol 295: H425H433, 2008.

382. Scragg JL, Dallas ML, Wilkinson JA, Varadi G, and Peers C. Carbon monoxide inhibits L-type $\mathrm{Ca}^{2+}$ channels via redox modulation of key cysteine residues by mitochondrial reactive oxygen species. J Biol Chem 283: 24412-24419, 2008.

383. Seiler A, Schneider M, Forster H, Roth S, Wirth EK, Culmsee C, Plesnila N, Kremmer E, Radmark O, Wurst W, Bornkamm GW, Schweizer U, and Conrad M. Glutathione peroxidase 4 senses and translates oxidative stress into 12/ 15-lipoxygenase dependent- and AIF-mediated cell death. Cell Metab 8: 237-248, 2008.

384. Selak MA, Armour SM, MacKenzie ED, Boulahbel H, Watson DG, Mansfield KD, Pan Y, Simon MC, Thompson $\mathrm{CB}$, and Gottlieb E. Succinate links TCA cycle dysfunction to oncogenesis by inhibiting HIF-alpha prolyl hydroxylase. Cancer Cell 7: 77-85, 2005.

385. Semenza GL. Oxygen-dependent regulation of mitochondrial respiration by hypoxia-inducible factor 1 . Biochem J 405: 1-9, 2007.

386. Semenza GL, Jiang BH, Leung SW, Passantino R, Concordet JP, Maire P, and Giallongo A. Hypoxia response elements in the aldolase A, enolase 1, and lactate dehydrogenase A gene promoters contain essential binding sites for hypoxia-inducible factor 1. J Biol Chem 271: 3252932537, 1996.

387. Shenouda SM, Widlansky ME, Chen K, Xu G, Holbrook M, Tabit CE, Hamburg NM, Frame AA, Caiano TL, Kluge MA, Duess MA, Levit A, Kim B, Hartman ML, Joseph L, Shirihai OS, and Vita JA. Altered mitochondrial dynamics contributes to endothelial dysfunction in diabetes mellitus. Circulation 124: 444-453, 2011.

388. Shi Q, Xu H, Kleinman WA, and Gibson GE. Novel functions of the alpha-ketoglutarate dehydrogenase complex may mediate diverse oxidant-induced changes in mitochondrial enzymes associated with Alzheimer's disease. Biochim Biophys Acta 1782: 229-238, 2008.

389. Shi T, Wang F, Stieren E, and Tong Q. SIRT3, a mitochondrial sirtuin deacetylase, regulates mitochondrial function and thermogenesis in brown adipocytes. J Biol Chem 280: 13560-13567, 2005.

390. Shimoda LA and Semenza GL. HIF and the lung: role of hypoxia-inducible factors in pulmonary development and disease. Am J Respir Crit Care Med 183: 152-156, 2011.

391. Sideris DP and Tokatlidis K. Trapping oxidative folding intermediates during translocation to the intermembrane space of mitochondria: in vivo and in vitro studies. Methods Mol Biol 619: 411-423, 2010. 
392. Skulachev VP, Antonenko YN, Cherepanov DA, Chernyak BV, Izyumov DS, Khailova LS, Klishin SS, Korshunova GA, Lyamzaev KG, Pletjushkina OY, Roginsky VA, Rokitskaya TI, Severin FF, Severina, II, Simonyan RA, Skulachev MV, Sumbatyan NV, Sukhanova EI, Tashlitsky VN, Trendeleva TA, Vyssokikh MY, and Zvyagilskaya RA. Prevention of cardiolipin oxidation and fatty acid cycling as two antioxidant mechanisms of cationic derivatives of plastoquinone (SkQs). Biochim Biophys Acta 1797: 878-889, 2010.

393. Soderdahl T, Enoksson M, Lundberg M, Holmgren A, Ottersen OP, Orrenius S, Bolcsfoldi G, and Cotgreave IA. Visualization of the compartmentalization of glutathione and protein-glutathione mixed disulfides in cultured cells. FASEB J 17: 124-126, 2003.

394. Someya S, Yu W, Hallows WC, Xu J, Vann JM, Leeuwenburgh C, Tanokura M, Denu JM, and Prolla TA. Sirt3 mediates reduction of oxidative damage and prevention of age-related hearing loss under caloric restriction. Cell 143: 802-812, 2010.

395. Song JY, Cha J, Lee J, and Roe JH. Glutathione reductase and a mitochondrial thioredoxin play overlapping roles in maintaining iron-sulfur enzymes in fission yeast. Eukaryot Cell 5: 1857-1865, 2006.

396. Sparagna GC and Lesnefsky EJ. Cardiolipin remodeling in the heart. J Cardiovasc Pharmacol 53: 290-301, 2009.

397. Srinivas V, Zhang LP, Zhu XH, and Caro J. Characterization of an oxygen/redox-dependent degradation domain of hypoxia-inducible factor alpha (HIF-alpha) proteins. Biochem Biophys Res Commun 260: 557-561, 1999.

398. St-Pierre J, Drori S, Uldry M, Silvaggi JM, Rhee J, Jager S, Handschin C, Zheng K, Lin J, Yang W, Simon DK, Bachoo $\mathrm{R}$, and Spiegelman BM. Suppression of reactive oxygen species and neurodegeneration by the PGC-1 transcriptional coactivators. Cell 127: 397-408, 2006.

399. Staniek K and Nohl H. H(2)O(2) detection from intact mitochondria as a measure for one-electron reduction of dioxygen requires a non-invasive assay system. Biochim Biophys Acta 1413: 70-80, 1999.

400. Stanley WC, Hernandez LA, Spires D, Bringas J, Wallace S, and McCormack JG. Pyruvate dehydrogenase activity and malonyl CoA levels in normal and ischemic swine myocardium: effects of dichloroacetate. J Mol Cell Cardiol 28: 905-914, 1996.

401. Starkov AA, Fiskum G, Chinopoulos C, Lorenzo BJ, Browne SE, Patel MS, and Beal MF. Mitochondrial alphaketoglutarate dehydrogenase complex generates reactive oxygen species. J Neurosci 24: 7779-7788, 2004.

402. Storozhevykh TP, Senilova YE, Persiyantseva NA, Pinelis VG, and Pomytkin IA. Mitochondrial respiratory chain is involved in insulin-stimulated hydrogen peroxide production and plays an integral role in insulin receptor autophosphorylation in neurons. BMC Neurosci 8: 84, 2007.

403. Stowe DF and Camara AK. Mitochondrial reactive oxygen species production in excitable cells: modulators of mitochondrial and cell function. Antioxid Redox Signal 11: 13731414, 2009.

404. Suliman HB, Carraway MS, Ali AS, Reynolds CM, Welty-Wolf $\mathrm{KE}$, and Piantadosi $\mathrm{CA}$. The $\mathrm{CO} / \mathrm{HO}$ system reverses inhibition of mitochondrial biogenesis and prevents murine doxorubicin cardiomyopathy. J Clin Invest 117: 3730-3741, 2007.

405. Sun J, Morgan M, Shen RF, Steenbergen C, and Murphy E. Preconditioning results in S-nitrosylation of proteins involved in regulation of mitochondrial energetics and calcium transport. Circ Res 101: 1155-1163, 2007.
406. Sun L, Xiao L, Nie J, Liu FY, Ling GH, Zhu XJ, Tang WB, Chen WC, Xia YC, Zhan M, Ma MM, Peng YM, Liu H, Liu $\mathrm{YH}$, and Kanwar YS. p66Shc mediates high-glucose and angiotensin II-induced oxidative stress renal tubular injury via mitochondrial-dependent apoptotic pathway. Am J Physiol Renal Physiol 299: F1014-F1025, 2011.

407. Susin SA, Lorenzo HK, Zamzami N, Marzo I, Snow BE, Brothers GM, Mangion J, Jacotot E, Costantini P, Loeffler M, Larochette N, Goodlett DR, Aebersold R, Siderovski DP, Penninger JM, and Kroemer G. Molecular characterization of mitochondrial apoptosis-inducing factor. Nature 397: 441-446, 1999.

408. Sutendra G, Bonnet S, Rochefort G, Haromy A, Folmes KD, Lopaschuk GD, Dyck JR, and Michelakis ED. Fatty acid oxidation and malonyl-CoA decarboxylase in the vascular remodeling of pulmonary hypertension. Sci Transl Med 2: 44ra58, 2010.

409. Swain SM, Whaley FS, and Ewer MS. Congestive heart failure in patients treated with doxorubicin: a retrospective analysis of three trials. Cancer 97: 2869-2879, 2003.

410. Szabo C, Ischiropoulos H, and Radi R. Peroxynitrite: biochemistry, pathophysiology and development of therapeutics. Nat Rev Drug Discov 6: 662-680, 2007.

411. Szczepanek K, Chen Q, Derecka M, Salloum FN, Zhang Q, Szelag M, Cichy J, Kukreja RC, Dulak J, Lesnefsky EJ, and Larner AC. Mitochondrial-targeted Signal transducer and activator of transcription 3 (STAT3) protects against ischemia-induced changes in the electron transport chain and the generation of reactive oxygen species. J Biol Chem 286: 29610-29620, 2011.

412. Szczepanek K, Chen Q, Larner AC, and Lesnefsky EJ. Cytoprotection by the modulation of mitochondrial electron transport chain: the emerging role of mitochondrial STAT3. Mitochondrion 2011 [Epub ahead of print]; DOI: 10.1016/ j.mito.2011.08.011

413. Takeshige $\mathrm{K}$ and Minakami S. NADH- and NADPHdependent formation of superoxide anions by bovine heart submitochondrial particles and NADH-ubiquinone reductase preparation. Biochem J 180: 129-135, 1979.

414. Tamura T, McMicken HW, Smith CV, and Hansen TN. Gene structure for mouse glutathione reductase, including a putative mitochondrial targeting signal. Biochem Biophys Res Commun 237: 419-422, 1997.

415. Tatoyan A and Giulivi C. Purification and characterization of a nitric-oxide synthase from rat liver mitochondria. J Biol Chem 273: 11044-11048, 1998.

416. Taylor ER, Hurrell F, Shannon RJ, Lin TK, Hirst J, and Murphy MP. Reversible glutathionylation of complex I increases mitochondrial superoxide formation. J Biol Chem 278: 19603-19610, 2003.

417. Teshima $\mathrm{Y}$, Akao $\mathrm{M}$, Jones SP, and Marban E. Uncoupling protein-2 overexpression inhibits mitochondrial death pathway in cardiomyocytes. Circ Res 93: 192200, 2003.

418. Thu VT, Kim HK, Ha SH, Yoo JY, Park WS, Kim N, Oh GT, and Han J. Glutathione peroxidase 1 protects mitochondria against hypoxia/reoxygenation damage in mouse hearts. Pflugers Arch 460: 55-68, 2010.

419. Tissenbaum HA and Guarente L. Increased dosage of a sir2 gene extends lifespan in Caenorhabditis elegans. Nature 410: 227-230, 2001.

420. Tong WH and Rouault T. Distinct iron-sulfur cluster assembly complexes exist in the cytosol and mitochondria of human cells. EMBO J 19: 5692-5700, 2000. 
421. Tong $X$, Ying J, Pimentel DR, Trucillo M, Adachi T, and Cohen RA. High glucose oxidizes SERCA cysteine-674 and prevents inhibition by nitric oxide of smooth muscle cell migration. J Mol Cell Cardiol 44: 361-369, 2008.

422. Tournier C, Hess P, Yang DD, Xu J, Turner TK, Nimnual A, Bar-Sagi D, Jones SN, Flavell RA, and Davis RJ. Requirement of JNK for stress-induced activation of the cytochrome c-mediated death pathway. Science 288: 870-874, 2000.

423. Tretter L and Adam-Vizi V. Alpha-ketoglutarate dehydrogenase: a target and generator of oxidative stress. Philos Trans R Soc Lond B Biol Sci 360: 2335-2345, 2005.

424. Tsang AH and Chung KK. Oxidative and nitrosative stress in Parkinson's disease. Biochim Biophys Acta 1792: 643-650, 2009.

425. Tuunanen $\mathrm{H}, \mathrm{Ukkonen} \mathrm{H}$, and Knuuti J. Myocardial fatty acid metabolism and cardiac performance in heart failure. Curr Cardiol Rep 10: 142-148, 2008.

426. Valente EM, Abou-Sleiman PM, Caputo V, Muqit MM, Harvey K, Gispert S, Ali Z, Del Turco D, Bentivoglio AR, Healy DG, Albanese A, Nussbaum R, GonzalezMaldonado R, Deller T, Salvi S, Cortelli P, Gilks WP, Latchman DS, Harvey RJ, Dallapiccola B, Auburger G, and Wood NW. Hereditary early-onset Parkinson's disease caused by mutations in PINK1. Science 304: 1158-1160, 2004.

427. Valle I, Alvarez-Barrientos A, Arza E, Lamas S, and Monsalve M. PGC-1alpha regulates the mitochondrial antioxidant defense system in vascular endothelial cells. Cardiovasc Res 66: 562-573, 2005.

428. van de Water B, Zoeteweij JP, and Nagelkerke JF. Alkylation-induced oxidative cell injury of renal proximal tubular cells: involvement of glutathione redox-cycle inhibition. Arch Biochem Biophys 327: 71-80, 1996.

429. Vander Heiden MG, Cantley LC, and Thompson CB. Understanding the Warburg effect: the metabolic requirements of cell proliferation. Science 324: 1029-1033, 2009.

430. Veatch JR, McMurray MA, Nelson ZW, and Gottschling DE. Mitochondrial dysfunction leads to nuclear genome instability via an iron-sulfur cluster defect. Cell 137: 12471258, 2009.

431. Venkatakrishnan P, Nakayasu ES, Almeida IC, and Miller RT. Absence of nitric-oxide synthase in sequentially purified rat liver mitochondria. J Biol Chem 284: 19843-19855, 2009.

432. Venkatakrishnan P, Nakayasu ES, Almeida IC, and Miller RT. Arginase activity in mitochondria-an interfering factor in nitric oxide synthase activity assays. Biochem Biophys Res Commun 394: 448-452, 2010.

433. Vessey DA and Lee KH. Inactivation of enzymes of the glutathione antioxidant system by treatment of cultured human keratinocytes with peroxides. J Invest Dermatol 100: 829-833, 1993.

434. Vianna CR, Huntgeburth M, Coppari R, Choi CS, Lin J, Krauss S, Barbatelli G, Tzameli I, Kim YB, Cinti S, Shulman GI, Spiegelman BM, and Lowell BB. Hypomorphic mutation of PGC-1beta causes mitochondrial dysfunction and liver insulin resistance. Cell Metab 4: 453-464, 2006.

435. Vieira HL, Belzacq AS, Haouzi D, Bernassola F, Cohen I, Jacotot E, Ferri KF, El Hamel C, Bartle LM, Melino G, Brenner C, Goldmacher V, and Kroemer G. The adenine nucleotide translocator: a target of nitric oxide, peroxynitrite, and 4-hydroxynonenal. Oncogene 20: 4305-4316, 2001.
436. Vilella F, Alves R, Rodriguez-Manzaneque MT, Belli G, Swaminathan S, Sunnerhagen P, and Herrero E. Evolution and cellular function of monothiol glutaredoxins: involvement in iron-sulphur cluster assembly. Comp Funct Genomics 5: 328-341, 2004.

437. Vives-Bauza C, Zhou C, Huang Y, Cui M, de Vries RL, Kim J, May J, Tocilescu MA, Liu W, Ko HS, Magrane J, Moore DJ, Dawson VL, Grailhe R, Dawson TM, Li C, Tieu K, and Przedborski S. PINK1-dependent recruitment of Parkin to mitochondria in mitophagy. Proc Natl Acad Sci U S A 107: 378-383, 2010.

438. von Ahsen O, Renken C, Perkins G, Kluck RM, BossyWetzel E, and Newmeyer DD. Preservation of mitochondrial structure and function after Bid- or Bax-mediated cytochrome c release. J Cell Biol 150: 1027-1036, 2000.

439. Walford GA, Moussignac RL, Scribner AW, Loscalzo J, and Leopold JA. Hypoxia potentiates nitric oxide-mediated apoptosis in endothelial cells via peroxynitrite-induced activation of mitochondria-dependent and -independent pathways. J Biol Chem 279: 4425-4432, 2004.

440. Wang G, Hamid T, Keith RJ, Zhou G, Partridge CR, Xiang $X$, Kingery JR, Lewis RK, Li Q, Rokosh DG, Ford R, Spinale FG, Riggs DW, Srivastava S, Bhatnagar A, Bolli R, and Prabhu SD. Cardioprotective and antiapoptotic effects of heme oxygenase- 1 in the failing heart. Circulation 121: 1912-1925, 2010.

441. Wang J, Pan S, and Berk BC. Glutaredoxin mediates Akt and eNOS activation by flow in a glutathione reductasedependent manner. Arterioscler Thromb Vasc Biol 27: 12831288, 2007.

442. Wang SK, Weaver JD, Zhang S, and Lei XG. Knockout of SOD1 promotes conversion of selenocysteine to dehydroalanine in murine hepatic GPX1 protein. Free Radic Biol Med 51: 197-204, 2011.

443. Wang Y, Qiao M, Mieyal JJ, Asmis LM, and Asmis R. Molecular mechanism of glutathione-mediated protection from oxidized low-density lipoprotein-induced cell injury in human macrophages: role of glutathione reductase and glutaredoxin. Free Radic Biol Med 41: 775-785, 2006.

444. Warburg O. On the origin of cancer cells. Science 123: 309314, 1956.

445. Wegrzyn J, Potla R, Chwae YJ, Sepuri NB, Zhang Q, Koeck T, Derecka M, Szczepanek K, Szelag M, Gornicka A, Moh A, Moghaddas S, Chen Q, Bobbili S, Cichy J, Dulak J, Baker DP, Wolfman A, Stuehr D, Hassan MO, Fu XY, Avadhani N, Drake JI, Fawcett P, Lesnefsky EJ, and Larner AC. Function of mitochondrial Stat3 in cellular respiration. Science 323: 793-797, 2009.

446. Westermann B. Molecular machinery of mitochondrial fusion and fission. J Biol Chem 283: 13501-13505, 2008.

447. Wilmanski J, Villanueva E, Deitch EA, and Spolarics Z. Glucose-6-phosphate dehydrogenase deficiency and the inflammatory response to endotoxin and polymicrobial sepsis. Crit Care Med 35: 510-518, 2007.

448. Wingert RA, Galloway JL, Barut B, Foott H, Fraenkel $\mathrm{P}$, Axe JL, Weber GJ, Dooley K, Davidson AJ, Schmid B, Paw $\mathrm{BH}$, Shaw GC, Kingsley P, Palis J, Schubert H, Chen O, Kaplan J, and Zon LI. Deficiency of glutaredoxin 5 reveals Fe-S clusters are required for vertebrate haem synthesis. Nature 436: 1035-1039, 2005.

449. Wink DA, Nims RW, Darbyshire JF, Christodoulou D, Hanbauer I, Cox GW, Laval F, Laval J, Cook JA, Krishna $\mathrm{MC}$, et al. Reaction kinetics for nitrosation of cysteine and glutathione in aerobic nitric oxide solutions at neutral $\mathrm{pH}$. 
Insights into the fate and physiological effects of intermediates generated in the $\mathrm{NO} / \mathrm{O}_{2}$ reaction. Chem Res Toxicol 7: 519-525, 1994.

450. Winterbourn CC and Hampton MB. Thiol chemistry and specificity in redox signaling. Free Radic Biol Med 45: 549561,2008

451. Wiswedel I, Gardemann A, Storch A, Peter D, and Schild L. Degradation of phospholipids by oxidative stress-exceptional significance of cardiolipin. Free Radic Res 44: 135-145, 2010.

452. Wong $\mathrm{CH}$, Bozinovski S, Hertzog PJ, Hickey MJ, and Crack PJ. Absence of glutathione peroxidase-1 exacerbates cerebral ischemia-reperfusion injury by reducing postischemic microvascular perfusion. J Neurochem 107: 241252, 2008.

453. Wong GH, Elwell JH, Oberley LW, and Goeddel DV. Manganous superoxide dismutase is essential for cellular resistance to cytotoxicity of tumor necrosis factor. Cell 58: 923-931, 1989.

454. Wood ZA, Poole LB, and Karplus PA. Peroxiredoxin evolution and the regulation of hydrogen peroxide signaling. Science 300: 650-653, 2003.

455. Wright GL, Maroulakou IG, Eldridge J, Liby TL, Sridharan V, Tsichlis PN, and Muise-Helmericks RC. VEGF stimulation of mitochondrial biogenesis: requirement of AKT3 kinase. FASEB J 22: 3264-3275, 2008.

456. Wu KK. Inducible cyclooxygenase and nitric oxide synthase. Adv Pharmacol 33: 179-207, 1995.

457. Wu S, Zhou F, Zhang Z, and Xing D. Mitochondrial oxidative stress causes mitochondrial fragmentation via differential modulation of mitochondrial fission-fusion proteins. FEBS J 278: 941-954, 2011.

458. Wu Z, Puigserver P, Andersson U, Zhang C, Adelmant G, Mootha V, Troy A, Cinti S, Lowell B, Scarpulla RC, and Spiegelman BM. Mechanisms controlling mitochondrial biogenesis and respiration through the thermogenic coactivator PGC-1. Cell 98: 115-124, 1999.

459. Xiong Y, Liu X, Lee CP, Chua BH, and Ho YS. Attenuation of doxorubicin-induced contractile and mitochondrial dysfunction in mouse heart by cellular glutathione peroxidase. Free Radic Biol Med 41: 46-55, 2006.

460. Xu Y, Zhang Z, Hu J, Stillman IE, Leopold JA, Handy DE, Loscalzo J, and Stanton RC. Glucose-6-phosphate dehydrogenase-deficient mice have increased renal oxidative stress and increased albuminuria. FASEB J 24: 609-616, 2010.

461. Yamashita R, Saito T, Satoh S, Aoki K, Kaburagi Y, and Sekihara H. Effects of dehydroepiandrosterone on gluconeogenic enzymes and glucose uptake in human hepatoma cell line, HepG2. Endocr J 52: 727-733, 2005.

462. Yang KS, Kang SW, Woo HA, Hwang SC, Chae HZ, Kim $\mathrm{K}$, and Rhee SG. Inactivation of human peroxiredoxin I during catalysis as the result of the oxidation of the catalytic site cysteine to cysteine-sulfinic acid. J Biol Chem 277: 38029-38036, 2002.

463. Yang $\mathrm{Y}$ and Loscalzo J. S-nitrosoprotein formation and localization in endothelial cells. Proc Natl Acad Sci U S A 102: 117-122, 2005

464. Yang Y, Song Y, and Loscalzo J. Regulation of the protein disulfide proteome by mitochondria in mammalian cells. Proc Natl Acad Sci U S A 104: 10813-10817, 2007.

465. Yant LJ, Ran Q, Rao L, Van Remmen H, Shibatani T, Belter JG, Motta L, Richardson A, and Prolla TA. The selenoprotein GPX4 is essential for mouse development and protects from radiation and oxidative damage insults. Free Radic Biol Med 34: 496-502, 2003.

466. Ye H and Rouault TA. Human iron-sulfur cluster assembly, cellular iron homeostasis, and disease. Biochemistry 49: 4945-4956, 2010.

467. Yet SF, Tian R, Layne MD, Wang ZY, Maemura K, Solovyeva M, Ith B, Melo LG, Zhang L, Ingwall JS, Dzau VJ, Lee ME, and Perrella MA. Cardiac-specific expression of heme oxygenase- 1 protects against ischemia and reperfusion injury in transgenic mice. Circ Res 89: 168-173, 2001.

468. Ying W. NAD + /NADH and NADP + /NADPH in cellular functions and cell death: regulation and biological consequences. Antioxid Redox Signal 10: 179-206, 2008.

469. Youle RJ and Narendra DP. Mechanisms of mitophagy. Nat Rev Mol Cell Biol 12: 9-14, 2011.

470. Young LJ and Caughey WS. Mitochondrial oxygenation of carbon monoxide. Biochem J 239: 225-227, 1986.

471. Zanella B, Giordano E, Muscari C, Zini M, and Guarnieri C. Nitric oxide synthase activity in rat cardiac mitochondria. Basic Res Cardiol 99: 159-164, 2004.

472. Zenebe WJ, Nazarewicz RR, Parihar MS, and Ghafourifar $\mathrm{P}$. Hypoxia/reoxygenation of isolated rat heart mitochondria causes cytochrome c release and oxidative stress; evidence for involvement of mitochondrial nitric oxide synthase. J Mol Cell Cardiol 43: 411-419, 2007.

473. Zhang CY, Baffy G, Perret P, Krauss S, Peroni O, Grujic D, Hagen T, Vidal-Puig AJ, Boss $\mathrm{O}$, Kim YB, Zheng XX, Wheeler MB, Shulman GI, Chan CB, and Lowell BB. Uncoupling protein-2 negatively regulates insulin secretion and is a major link between obesity, beta cell dysfunction, and type 2 diabetes. Cell 105: 745-755, 2001.

474. Zhang J, Jin B, Li L, Block ER, and Patel JM. Nitric oxideinduced persistent inhibition and nitrosylation of active site cysteine residues of mitochondrial cytochrome-c oxidase in lung endothelial cells. Am J Physiol Cell Physiol 288: C840C849, 2005.

475. Zhang J and Piantadosi CA. Mitochondrial oxidative stress after carbon monoxide hypoxia in the rat brain. J Clin Invest 90: 1193-1199, 1992.

476. Zhang Z, Liew CW, Handy DE, Zhang Y, Leopold JA, Hu J, Guo L, Kulkarni RN, Loscalzo J, and Stanton RC. High glucose inhibits glucose-6-phosphate dehydrogenase, leading to increased oxidative stress and beta-cell apoptosis. FASEB J 24: 1497-1505, 2010.

477. Zhao $Y$, Jin J, Hu Q, Zhou HM, Yi J, Yu Z, Xu L, Wang X, Yang Y, and Loscalzo J. Genetically encoded fluorescent sensors for intracellular NADH detection. Cell Metab 14: 555-566, 2011.

478. Zheng J, Fang J, Yin YJ, Wang XC, Ren AJ, Bai J, Sun XJ, Yuan WJ, and Lin L. Leptin protects cardiomyocytes from serum-deprivation-induced apoptosis by increasing anti-oxidant defence. Clin Exp Pharmacol Physiol 37: 955$962,2010$.

479. Zhong L, D'Urso A, Toiber D, Sebastian C, Henry RE, Vadysirisack DD, Guimaraes A, Marinelli B, Wikstrom JD, Nir T, Clish CB, Vaitheesvaran B, Iliopoulos O, Kurland I, Dor Y, Weissleder R, Shirihai OS, Ellisen LW, Espinosa JM, and Mostoslavsky R. The histone deacetylase Sirt6 regulates glucose homeostasis via Hif1alpha. Cell 140: 280-293, 2010.

480. Zhou Q, Lam PY, Han D, and Cadenas E. c-Jun N-terminal kinase regulates mitochondrial bioenergetics by modulating pyruvate dehydrogenase activity in primary cortical neurons. J Neurochem 104: 325-335, 2008. 
481. Zorov DB, Filburn CR, Klotz LO, Zweier JL, and Sollott SJ. Reactive oxygen species (ROS)-induced ROS release: a new phenomenon accompanying induction of the mitochondrial permeability transition in cardiac myocytes. J Exp Med 192: 1001-1014, 2000.

Address correspondence to: Dr. Joseph Loscalzo Cardiovascular Division Department of Medicine Brigham and Women's Hospital Harvard Medical School 75 Francis St. Boston, MA 02115

E-mail: jloscalzo@partners.org

Date of first submission to ARS Central, June 22, 2011; date of final revised submission, December 6, 2011; date of acceptance, December 6, 2011.

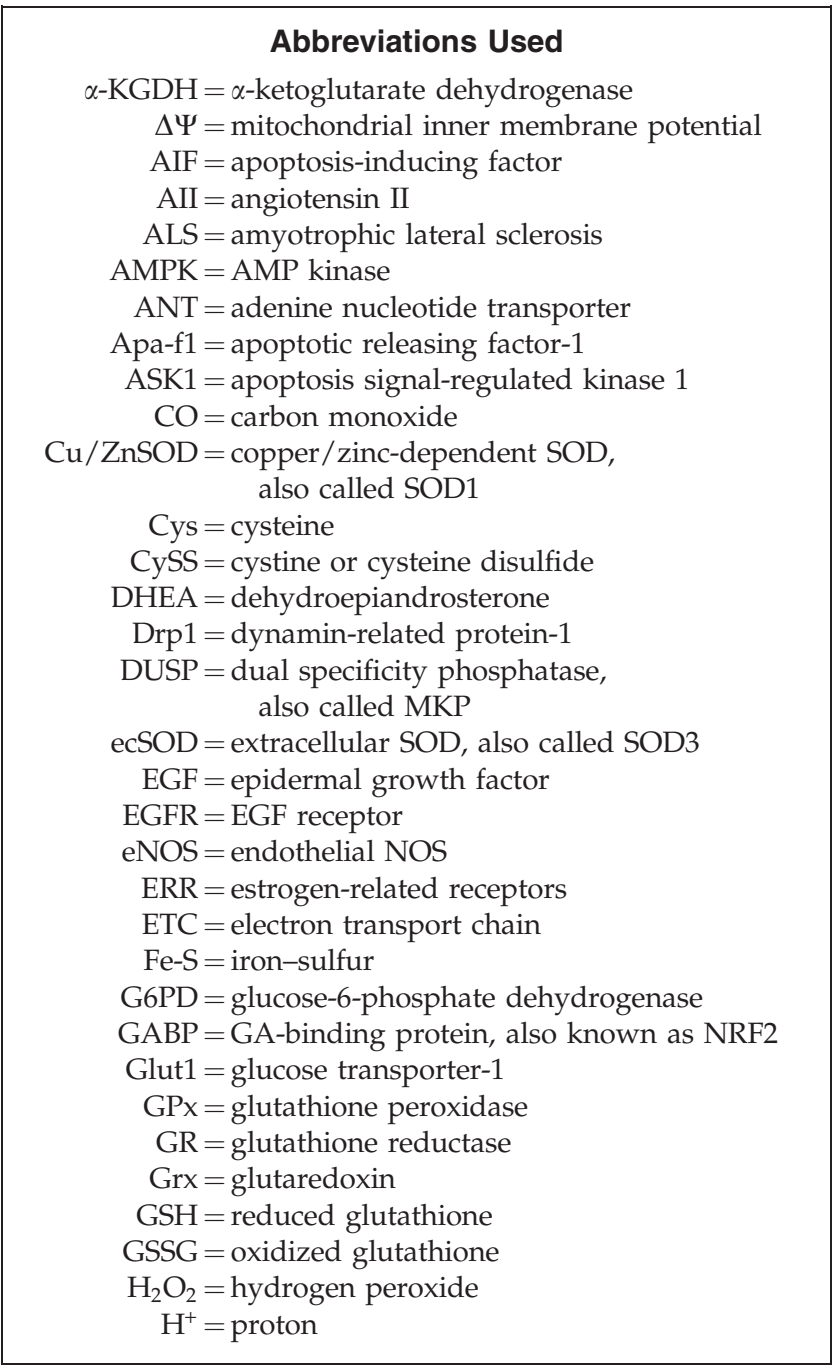

HIF-1 = hypoxia-inducible factor-1

$\mathrm{ICDH}=$ isocitrate dehydrogenase

iNOS $=$ inducible NOS

ISCU1/2 = iron-sulfur cluster assembly proteins 1,2 $\mathrm{JNK}=\mathrm{c}$-jun $\mathrm{N}$-terminal kinase

$\mathrm{LC}=$ liquid chromatography

$\mathrm{LDH}=$ lactate dehydrogenase

$\mathrm{LDHA}=$ lactate dehydrogenase $\mathrm{A}$

$\mathrm{LDHB}=$ lactate dehydrogenase $\mathrm{B}$

LPS $=$ lipopolysaccharide

MAPK $=$ mitogen-activated protein kinase

$\mathrm{MDH}=$ malate dehydrogenase

$\mathrm{MnSOD}=$ manganese-dependent SOD, also known as SOD2

$\mathrm{MPT}=$ mitochondrial permeability transition

$\mathrm{MS} / \mathrm{MS}=$ tandem mass spectrometry

$\mathrm{mtDNA}=$ mitochondrial DNA

$\mathrm{nNOS}=$ neuronal NOS

$\mathrm{NNT}=$ nicotinamide nucleotide transhydrogenase

$\mathrm{NO}=$ nitric oxide

$\mathrm{NOS}=$ nitric oxide synthase

$\mathrm{NOX}=\mathrm{NADPH}$-dependent oxidase

$\mathrm{NQ1}=\mathrm{NADH}$ :quinone oxidoreductase 1

$\mathrm{NRF}=$ nuclear respiratory factor

$\mathrm{O}_{2}^{\bullet-}=$ superoxide anion

$\mathrm{PDH}=$ pyruvate dehydrogenase

$\mathrm{PDK}=\mathrm{PDH}$ kinase

PGC-1 $=$ peroxisome proliferator-activated receptor- $\gamma$ coactivator -1

PHD $=$ prolyl hydroxylase

PI3K = phosphatidylinositol 3 kinase

PINK $=$ PTEN-induced kinase- 1

PIP3 = phosphatidylinositol 3,4,5 phosphate

Prx $=$ peroxiredoxin

PTEN $=$ phosphatase and tensin homolog

$\mathrm{Q}=$ ubiquinone

$\mathrm{RNS}=$ reactive nitrogen species

ROS $=$ reactive oxygen species

rxYFP $=$ redox-sensitive yellow fluorescent protein

$\mathrm{SAPK}=$ stress-activated MAPK

$\mathrm{Sec}=$ selenocysteine

SERCA = sarcoplasmic-endoplasmic reticulum calcium ATPase

$\mathrm{sGC}=$ soluble guanylyl cyclase

SIRT $=$ sirtuins

$\mathrm{SOD}=$ superoxide dismutase

Srx $=$ sulfiredoxin

$\mathrm{TAC}=$ transverse aortic constriction

TCA $=$ tricarboxylic acid

TERT $=$ telomerase reverse transcriptase

TFAM $=$ transcription factor $\mathrm{A}$

$\mathrm{TNF}=$ tumor necrosis factor

TRAF $=$ TNF-receptor-associated adaptor proteins

$\operatorname{Trx}=$ thioredoxin

$\operatorname{Tr} x \mathrm{R}=$ thioredoxin reductase

$\mathrm{TZD}=$ thiazolidinediones

$\mathrm{UCP}=$ uncoupling proteins

$\mathrm{VDAC}=$ voltage-dependent anion channel

$\mathrm{VHL}=$ von Hippel-Lindau tumor suppressor 